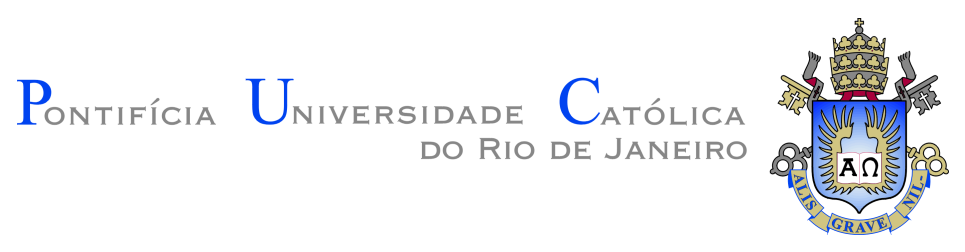

Rouhollah Ebrahimi

\title{
Extreme Value Statistics of Random Normal \\ Matrices
}

Tese de Doutorado

Thesis presented to the Programa de Pós-graduação em Física of PUC-Rio in partial fulfillment of the requirements for the degree of Doutor em Ciências - Física.

Advisor : Prof. Hiroshi Nunokawa

Co-advisor: Prof. Stefan Zohren

Rio de Janeiro

October 2017 


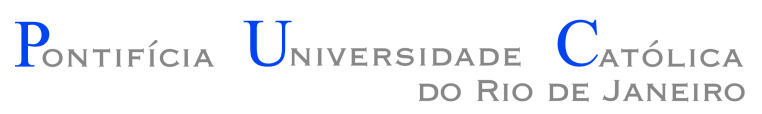

Rouhollah Ebrahimi

\section{Extreme Value Statistics of Random Normal \\ Matrices}

Thesis presented to the Programa de Pós-graduação em Física of PUC-Rio in partial fulfillment of the requirements for the degree of Doutor em Ciências - Física. Approved by the undersigned Examination Committee.

Prof. Hiroshi Nunokawa

Advisor

Departamento de Física PUC-Rio

Prof. Stefan Zohren

Co-advisor

University of Oxford

Prof. Alexei Margalhaes Veneziani

Universidade Federal do ABC

Prof. Carlos Tomei

Departamento de Matemática - PUC-Rio

Prof. José Abdalla Helayël-Neto

CBPF

Prof. Domingos Humberto Urbano Marchetti

USP

Prof. Luis Esteban Oxman

UFF

Prof. Márcio da Silveira Carvalho

Vice Dean of Graduate Studies

Centro Técnico Científico - PUC-Rio

Rio de Janeiro, October the 25th, 2017 
All rights reserved.

\section{Rouhollah Ebrahimi}

Graduated in Physics at Shahed University in 2004 and obtained his M.Sc. degree in Physics from Sharif University of Technology in 2008.

Bibliographic data

Ebrahimi, Rouhollah

Extreme Value Statistics of Random Normal Matrices / Rouhollah Ebrahimi; advisor: Hiroshi Nunokawa; co-advisor: Stefan Zohren. - Rio de janeiro: PUC-Rio, Departamento de Física, 2017.

v., 135 f: il. color. ; $30 \mathrm{~cm}$

Tese (doutorado) - Pontifícia Universidade Católica do Rio de Janeiro, Departamento de Física.

Inclui bibliografia

1. Física - Teses. 2. Matrizes Aleatórias Normais;. 3. Estatísticas de Valor Extremo;. 4. Universalidade;. 5. Polinômios Ortogonais.. I. Nunokawa, Hiroshi. II. Zohren, Stefan. III. Pontifícia Universidade Católica do Rio de Janeiro. Departamento de Física. IV. Título. 
Dedicated to my mother and sister whose support has been very precious, and to the memory of my father. 


\section{Acknowledgments}

I would like to thank my advisor, Hiroshi Nunokawa, for all his help and guidance during my Ph.D. studies. My sincere gratitude also goes to co-advisor, Stefan Zohren, who introduced me to the fascinating world of random matrices. That was a great chance for me to benefit form his deep and broad knowledge in physics and mathematics. I also appreciate his patience and remarkable character which has been an exceptional inspiration throughout my graduate life.

Special thanks to Carlos Tomei, at department of mathematics at PUC-Rio, whose presence and support helped me through difficulties. Besides being a great mathematician, he has a wonderful personality.

I would also like to thank the constant support of Welles Morgado and José Helayël.

With respect to my visit at institute of mathematics and statistics (IME), at university of São Paulo, I am particularly grateful to Anatoli Iambartsev, who provided a pleasant environment for me to work.

Financial support through CAPES, CNPq and CCPG (PUC-Rio) is kindly acknowledged. 


\section{Abstract}

Ebrahimi, Rouhollah; Nunokawa, Hiroshi (Advisor); Zohren, Stefan (Co-Advisor). Extreme Value Statistics of Random Normal Matrices. Rio de Janeiro, 2017. 135p. Tese de doutorado Departamento de Física, Pontifícia Universidade Católica do Rio de Janeiro.

With diverse applications in mathematics, physics, and finance, Random Matrix Theory (RMT) has recently attracted a great deal of attention. While Hermitian RMT is of special importance in physics because of the Hermiticity of operators associated with observables in quantum mechanics, non-Hermitian RMT has also attracted a considerable attention, in particular because they can be used as models for dissipative or open physical systems. However, due to the absence of a simplifying symmetry, the study of non-Hermitian random matrices is, in general, a difficult task. A special subset of non-Hermitian random matrices, the so-called random normal matrices, are interesting models to consider, since they offer more symmetry, thus making them more amenable to analytical investigations. By definition, a normal matrix $M$ is a square matrix which commutes with its Hermitian adjoint, i.e., $\left[M, M^{\dagger}\right]=0$. In this thesis, we present a novel derivation of extreme value statistics (EVS) of Hermitian random matrices, namely the approach of orthogonal polynomials, to normal random matrices and $2 D$ Coulomb gases in general. The strength of this approach is its physical and intuitive understanding. Firstly, this approach provides an alternative derivation of results in the literature. Precisely speaking, we show convergence of the rescaled eigenvalue with largest modulus of a Ginibre ensemble to a Gumbel distribution, as well as universality for an arbitrary radially symmetric potential which meets certain conditions. Secondly, it is shown that this approach can be generalised to obtain convergence of the eigenvalue with smallest modulus and its universality at the finite inner edge of the eigenvalue support. One interesting aspect of this work is the fact that we can use standard techniques from Hermitian random matrices to obtain the EVS of non-Hermitian random matrices.

\section{Keywords}

Random Normal Matrices; Extreme Value Statistics; Universality; Orthogonal Polynomials. 


\section{Resumo}

Ebrahimi, Rouhollah; Nunokawa, Hiroshi; Zohren, Stefan. Estatísticas de Valor Extremo de Matrizes Aleatórias Normais. Rio de Janeiro, 2017. 135p. Tese de Doutorado - Departamento de Física, Pontifícia Universidade Católica do Rio de Janeiro. Com diversas aplicações em matemática, física e finanças, Teoria das Matrizes Aleatórias (RMT) recentemente atraiu muita atenção. Enquanto o RMT Hermitiano é de especial importância na física por causa da Hermenticidade de operadores associados a observáveis em mecânica quântica, O RMT não-Hermitiano também atraiu uma atenção considerável, em particular porque eles podem ser usados como modelos para sistemas físicos dissipativos ou abertos. No entanto, devido à ausência de uma simetria simplificada, o estudo de matrizes aleatórias não-Hermitianas é, em geral, uma tarefa difícil. Um subconjunto especial de matrizes aleat órias não-Hermitianas, as chamadas matrizes aleat'orias normais, são modelos interessantes a serem considerados, uma vez que oferecem mais simetria, tornando-as mais acessíveis às investigções analíticas. Por definição, uma matriz normal $M$ é uma matriz quadrada que troca com seu adjunto Hermitiano. Nesta tese, amplicamos a derivação de estatísticas de valores extremos (EVS) de matrizes aleatórias Hermitianas, com base na abordagem de polinômios ortogonais, em matrizes aleatórias normais e em gases Coulomb $2 D$ em geral. A força desta abordagem a sua compreensão física e intuitiva. Em primeiro lugar, essa abordagem fornece uma derivação alternativa de resultados na literatura. Precisamente falando, mostramos a convergência do autovalor redimensionado com o maior módulo de um conjunto de Ginibre para uma distribuição de Gumbel, bem como a universalidade para um potencial arbitrário radialmente simtérico que atenda certas condições. Em segundo lugar, mostra-se que esta abordagem pode ser generalizada para obter a convergência do autovalor com menor módulo e sua universalidade no limite interno finito do suporte do autovalor. Um aspecto interessante deste trabalho é o fato de que podemos usar técnicas padrão de matrizes aleatórias Hermitianas para obter o EVS de matrizes aleatórias não Hermitianas.

\section{Palavras-chave}

Matrizes Aleatórias Normais; Estatísticas de Valor Extremo; Universalidade; Polinômios Ortogonais. 


\section{Table of contents}

$\begin{array}{ll}\text { List of figures } & 10\end{array}$

$\begin{array}{ll}\text { List of tables } & 12\end{array}$

$\begin{array}{lll}1 & \text { Introduction } & 15\end{array}$

2 Basic Notions of Probability Theory and Statistics 19

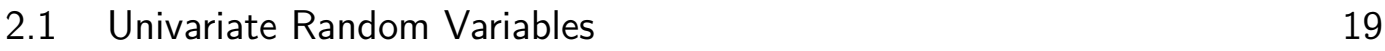

2.1.1 Special Probability Distributions $\quad 22$

2.1.2 Moment-Generating Functions 24

2.2 Multivariate Random Variables 26

2.3 Convergence of Sequence of Random Variables 27

2.3.1 Convergence in Probability and Weak Law of Large Numbers 27

2.3.2 Convergence in Distribution and Central Limit Theorem 29

$\begin{array}{lll}2.4 & \text { Tail Distribution } & 31\end{array}$

2.4.1 Concentration Inequalities and Tail Bounds 31

2.4.2 Classification of tails 34

$3 \quad$ Asymptotic Models of Classical Extreme Value Theory 37

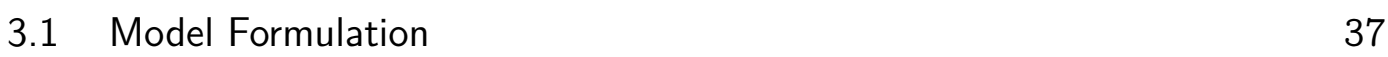

3.2 The Three Types of Extreme Value Distributions 39

3.3 The Generalized Extreme Value Distribution for Maxima 40

3.4 The Generalized Extreme Value Distribution for Minima 44

3.5 Domains of Attraction 45

4 Introduction to Random Matrix Theory $\quad 49$

$\begin{array}{lll}4.1 & \text { Motivation } & 49\end{array}$

4.2 Classical Ensembles of Random Matrices 50

4.3 Universality of Random Matrix Theories $\quad 54$

4.3.1 Level Spacing Distribution $\quad 55$

$\begin{array}{ll}\text { 4.3.2 Eigenvalue Distribution } & 57\end{array}$

$5 \quad$ Gaussian Unitary Ensemble $\quad 58$

5.1 Spectral Representation of the Partition Function of GUE 58

5.2 Methods of Analysis the Large $N$ Limit $\quad 60$

5.2.1 Saddle Point Method for GUE 60

5.2.2 Resolvent Formalism for Unitary Ensembles with Arbitrary Potential 63

5.2.3 Orthogonal Polynomials Method for Unitary Ensemble with Arbitrary Potential 69

5.2.4 Results of Orthogonal Polynomials Method for GUE 72 
$\begin{array}{lll}6 & \text { Ginibre Ensemble } & 74\end{array}$

$\begin{array}{lll}6.1 & \text { Resolvent Formalism } & 74\end{array}$

6.2 Diagrammatic Method for Hermitian Random Matrices 78

6.3 Diagrammatic Method for Non-Hermitian Random Matrices 81

7 Random Normal Matrix Ensembles $\quad 84$

7.1 Eigenvalue Representation of the Partition Function 84

$\begin{array}{lll}7.2 & \text { Saddle Point Method } & 85\end{array}$

$\begin{array}{lll}7.3 & \text { Orthogonal Polynomials Method } & 89\end{array}$

8 Extreme Value Statistics of GUE $\quad 93$

8.1 Tracy-Widom Law for the Classical Gaussian Ensembles 93

8.2 Orthogonal Polynomials Approach to the EVS of GUE 96

8.3 The String Equation in the Presence of a cut-off 98

8.4 Double Scaling Limit of the String Equation 99

9 Extreme Value Statistics of Random Normal Matrices 104

9.1 EVS of Gaussian Random Normal Matrices at the Outer Edge 104

9.1.1 First Approach Using Asymptotics of Regularized Incomplete Gamma $\begin{array}{ll}\text { Function } & 106\end{array}$

9.1.2 Second Approach Using Asymptotics of Error Function 112

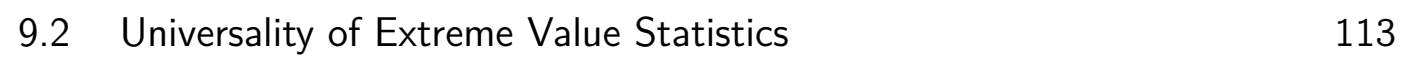

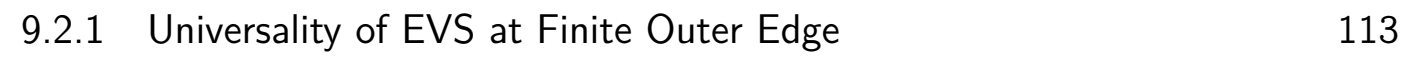

$\begin{array}{ll}\text { 9.2.2 Universality of EVS at Finite Inner Edge } & 115\end{array}$

10 Outlook and Conclusion 118

$\begin{array}{lll}\text { A Regularly and Slowly Varying Functions } & 121\end{array}$

B Asymptotic Expansions of Regularized Incomplete Gamma $\begin{array}{ll}\text { Functions } & 124\end{array}$

C Euler-MacLaurin Summation Formula 126

$\begin{array}{ll}\text { Bibliography } & 128\end{array}$

$\begin{array}{ll}\text { Bibliography } & 128\end{array}$ 


\section{List of figures}

Figure 2.1 Relation between cdf and pdf. $\quad 21$

Figure 2.2 Probability of finding $X$ in the interval $[a, b] . \quad 21$

Figure 2.3 The function $\Lambda(t)$ plotted against the line $y=x t$, in the case when $\Lambda^{\prime}(0)=0$. The point $\tau$ marks the value of $t$ at which $x t-\Lambda(t)$ is a maximum, and this maximum is denoted $\Lambda^{*}(x)$.

Figure 2.4 Tail of Pareto distribution with $x_{m}=1$, and for different values of tail index $\alpha$.

Figure 3.1 Generalized extreme value densities, all with $\mu=0$ and $\sigma=1$, and different extreme value index $\xi$. Asterisks mark endpoints of the supports.

Figure 4.1 Diffusion rate of neutrons as a function of the energy. The distance between neighbouring energy levels is denoted by $s$.

Figure 4.2 Wigner surmise for level spacing probability distribution. 57

Figure 5.1 Wigner's semi-circle law for GUE.

Figure 5.2 Repulsion of the Coulomb gas of eigenvalues.

Figure 5.3 Discontinuity of the resolvent across the branch cut.

Figure 5.4 Counterclockwise contours $C, C_{z}$, and $C_{\infty}$, and the cut $\mathcal{S}=\left[c_{-}, c_{+}\right]$in the complex $u$-plane.

Figure $6.1 \quad\left(Z^{-1}\right)_{a b}$ depicted as a line between $a$ and $b$.

Figure 6.2 The propagator $\mathbb{E}\left[M_{a b} M_{c d}\right]$ represented as a double arc, joining $a$ with $d$, and $b$ with $c$, respectively.

Figure 6.3 Diagrammatical representation of Eq. (6-17). 80

Figure 6.4 Diagrammatical representation of self-energy $\Sigma$.

Figure 6.5 Diagrammatical representation of the first DysonSchwinger equation.

Figure 6.6 Diagrammatical representation of the second DysonSchwinger equation.

Figure 6.7 Circular law for the Ginibre ensemble. 
Figure 7.1 Left: Distribution of eigenvalues of a $1000 \times 1000$ Ginibre matrix equivalent to a Coulomb gas with Gaussian potential $V(r)=r^{2}$. The eigenvalues density is given by the circular law. Right: Distribution of eigenvalues of a $1000 \times$ 1000 normal random matrix with potential $V(r)=r^{2}-r$ obtained using Monte-Carlo methods. The eigenvalue density has support on a ring.

Figure 8.1 The average eigenvalue density of a Gaussian random matrix ensemble $\rho(\lambda)$ with a compact support $[-\sqrt{2}, \sqrt{2}]$. The eigenvalue with the largest modulus has an expectation value $\mathbb{E}\left[\lambda_{\max }\right]=\sqrt{2}$ and its distribution close to this expectation value has, over a scale $\mathcal{O}\left(N^{-2 / 3}\right)$, obeys Tracy-Widom law (red solid line). However, over a scale $\mathcal{O}(1)$, the distribution has large deviation tails shown by solid green (left large deviations) and solid blue (right large deviations) lines.

Figure 8.2 Largest eigenvalue densities $f_{\beta}(Y) \equiv \mathrm{d} F_{\beta}(Y) / \mathrm{d} Y$ for $\beta=1,2,4$.

Figure 9.1 Shown is the function $F_{N}(Y)$ for increasing values of $N$, as well as the limiting case $F(Y)=\exp (-\exp (-Y))$. The function $F_{N}(Y)$ is obtained by numerically evaluating (9-11) with $y$ replaced with its scaling relation (9-18).

Figure 9.2 Comparison of the finite $N$ expression $F_{N}(Y)$ as given in (9-13) with the empirical cumulative probability function obtained from sampling 1000 Ginibre matrices of size $N=200$ and $N=1000$ respectively. 


\section{List of tables}

Table 4.1 Gaussian and circular ensembles. 


\section{List of Abreviations}

:= The quantity on the left is defined to be equal to the quantity on the right.

$=: \quad$ The quantity on the right is defined to be equal to the quantity on the left.

$\sim \quad f(n) \underset{n \rightarrow \infty}{\sim} g(n)$, iff $\lim _{n \rightarrow \infty} f(n) / g(n)=1$.

$O \quad f(n)=O(g(n))$ as $n \rightarrow \infty$, iff there exists $M \in \mathbb{R}^{+}$and $n_{0} \in \mathbb{R}$ such that $|f(n)| \leq|g(n)|, \forall n \geq n_{0}$.

$o \quad f(n)=o(g(n))$ iff for every $\epsilon \in \mathbb{R}^{+}$, there exists a constant $N$ such that $|f(n)| \leq \epsilon|g(n)|, \forall n \geq N$.

$\equiv \quad$ The quantities on the both sides are equivalent.

$\rightsquigarrow \quad$ Convergence in distribution.

$\overline{\mathbb{R}} \quad$ Extended real number system, $\overline{\mathbb{R}}=\mathbb{R} \cup\{ \pm \infty\}$.

$\mathbb{N}_{0} \quad \mathbb{N} \cup\{0\}$.

f Principal value integral. 
If there is anything that can bind the heavenly mind of man to this dreary exile of our earthly home and can reconcile us with our fate, so that one can enjoy living, then, it is verily, the enjoyment of the mathematical sciences and astronomy.

J. Kepler, in a letter to J. Batrsch. 


\section{Introduction}

I found it difficult, to keep my mind from the wandering into the magical world of random matrices.

F. J. Dyson

Random Matrices have fascinated both mathematicians and physicists, since they were first introduced in mathematical statistics by Wishart [1]. Then, this subject gained prominence when Wigner introduced the concept of statistical distribution of nuclear energy levels in 1950 [2]. The mathematical foundations of Random Matrix Theory (RMT) were stablished in a series of beautiful papers by Dyson [3-7] in which he introduced the classification of random matrix ensembles according to their invariance properties under time reversal. RMT has since become a rich and flourishing subject, with an enormous and growing literature and applications to numerous areas of physics and even to pure mathematics.

Being an active branch of mathematical research per se, RMT has turned out to be a quite powerful tool with a wide breadth of applications in many disciplines of science, engineering and finance [8]. Examples include quantum mechanics [9], condensed matter physics [10], classical and quantum optics [11] and wireless communication [12], to name just a few.

Moreover, different approaches to RMT provide links to other areas of mathematics, which makes it an interesting field of research in its own right; e.g., the Coulomb gas method can be interpreted in terms of algebraic geometry and loop equations can be solved using topological recursion, while the orthogonal polynomials technique makes connection with integrable systems [13].

An interesting subject which lies at the interface between RMT, proba- 
bility theory and statistics, is the Extreme Value Statistics (EVS) of random matrices. In this thesis we focus on the EVS of random normal matrices.

In part I of this thesis, we review the probabilistic aspects of the subject. In Ch. 2 we have a brief look at the basic concepts of probability theory which provide the theoretical foundations for the rest of the thesis. Our focus is on the continuous random variables. Specially, we introduce two different kinds of convergence, i.e., convergence in probability and convergence in distribution, which are necessary tools during the subsequent discussions in Ch. 3. Furthermore, the notion of tail distributions and their classification are reviewed in this chapter.

Ch. 3 is concerned with the central result of classical extreme value theory, the extermal types theorem, which specifies the possible forms for the limiting distribution of maxima (minima) in sequence of iid random variables. The asymptotic formulation of classical extreme value statistics is discussed, and then three classes of extreme value distributions along with their tail behaviour, are studied.

Part II is devoted to the study of foundations of RMT. After giving motivation for random matrix models in Ch. 4, We introduce the three classical ensembles of random matrices. Then, the notion of universality as a reason for wide range applications of RMT is discussed. In Ch. 5 we focus on the Gaussian unitary ensemble (GUE): first, we derive the joint probability distribution of eigenvalues and subsequently, we present two methods of analysis of large $N$ limit of GUE; i.e., the saddle point method and the method of orthogonal polynomials. The generalization of these methods for an arbitrary potential are also presented. In Ch. 6 we show how one can implement complex analysis and diagrammatic methods to obtain the eigenvalue density for the the case of Ginibre ensemble. In the final chapter of this part, Ch. 7, we introduce the ensemble of random normal matrices as a special case of non-Hermitian random matrices, and we show how their symmetry can be employed to apply the aforementioned methods, i.e., saddle point and orthogonal polynomials method in this case. 
Chapters 1-7 of the first two parts as well as Ch. 8 of the last part of this thesis include introductory and review material which don't include new results and references to the original works are provided. In Ch. 8 we review the celebrated Tracy-Widom law for the probability distribution of the GUE. The approach used is based on a method implemented in [14] which makes connection to integrable systems.

The novel part of this thesis is presented in Chapter 9, which is based on the following article:

"On the extreme value statistics of normal random matrices", R. Ebrahimi, S. Zohren, [arXiv: 1704.07488v1].

In this article we introduce a new approach to calculate the EVS of random normal matrices. It is both intuitive and general, allowing us to give a simplified proof of results known in the literature as well as new results. The strength of this approach lies in the fact that it provides a method to derive the EVS of random normal matrices for arbitrary ring distributions, both at the outer and inner (finite) edges of the eigenvalue support. Furthermore, one, in principle, is able to use this method to calculate finite $N$ corrections to the EVS of normal matrix models. 


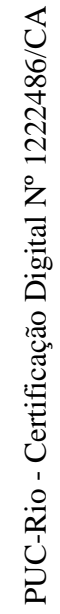




\section{2}

\section{Basic Notions of Probability Theory and Statistics}

The true logic of this world lies in probability theory.

$$
\text { J. C. Maxwell }
$$

This chapter, which has a brief look on some fundamental concepts and methods of probability theory and statistics, is included for this thesis to be (almost) self-contained and provides the theoretical basis for whole thesis. Most of the material of the first two sections can be found in any standard textbook on the subject, such as [15-19]. Furthermore, the last two sections of this chapter are devoted to discuss convergence of sequence of random variables and tail distribution, which will be of frequent use in the next chapter.

\section{1}

\section{Univariate Random Variables}

The basic ingredients of a statistical model are the following:

- A sample space $\Omega$, which is the set of all possible outcomes.

- A $\sigma$-algebra $\mathcal{F}$, which is a collection of all the events.

- A real-valued probability measure function $\mathbb{P}: \mathcal{F} \rightarrow[0,1]$ which assigns probabilities to the events, satisfying the Kolmogorov axioms [15]:

1. $0 \leq \mathbb{P}(E) \leq 1, \quad \forall E \in \mathcal{F}$.

2. $\mathbb{P}(\Omega)=1$.

3. $\mathrm{P}\left(\bigcup_{i} E_{i}\right)=\sum_{i} \mathrm{P}\left(E_{i}\right)$, for every (finite or infinite) sequence of events $E_{1}, E_{2}, \ldots$ 
The triplet $(\Omega, \mathcal{F}, \mathbb{P})$ is a probability space that models a real-world process (or "experiment") consisting of states that occur randomly.

A random variable $X: \Omega \rightarrow S$ is a real-valued measurable function from the sample space $\Omega$ to another measurable space $S$, called the state space. Although $X$ is usually a real-valued function $(S \subseteq \mathbb{R})$, it does not return a probability. The probabilities of different outcomes or sets of outcomes (events) are already given by the probability measure $\mathbb{P}$ with which $\Omega$ is equipped. Rather, $X$ describes some numerical property that outcomes in $\Omega$ may have. Recording all the probabilities of output ranges of a real-valued random variable $X$, yields the probability distribution function of $X$. When the image (or range) of $X$ is finite or countably infinite, the random variable is called a discrete random variable and its probability distribution function can be described by a probability mass function which assigns a probability to each value in the image of $X$. More precisely, the probability mass function $p_{X}: S \rightarrow[0,1]$ is defined as

$$
p_{X}(x) \equiv \mathbb{P}(X=x):=\mathbb{P}(\{\omega \in \Omega: X(\omega)=x\}) .
$$

Most of the random variables to which extreme value methods (see next chapter) are applied, are continuous random variables. This means they have a sample space $\Omega$ that is continuous. ${ }^{1}$ Because of the continuity, it is not possible to assign probabilities to all possible values of the random variable in a meaningful way. Loosely speaking, there are simply too many possible values on a continuous scale. Instead, probability distributions can be specified by their cumulative distribution function (cdf), or simply distribution function $P_{X}: \mathbb{R} \rightarrow[0,1]$, defined as

$$
P_{X}(x):=\mathbb{P}(X \leq x), \quad \forall x \in \mathbb{R},
$$

where the right-hand side represents the probability that the random variable $X$ takes on a value less than or equal to $x$. For the axioms of probability to be satisfied, $P_{X}$ must satisfy the following conditions:

1. Monotonicity: if $x \leq y$, then $P_{X}(x) \leq P_{X}(y)$.

\footnotetext{
${ }^{1}$ Hereafter, we suppose $\Omega=\mathbb{R}$, unless indicated otherwise.
} 


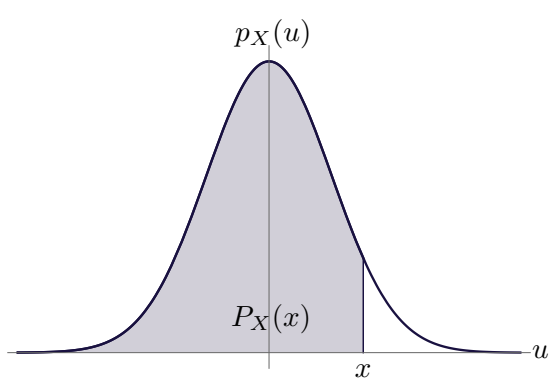

Figure 2.1: Relation between cdf and pdf.

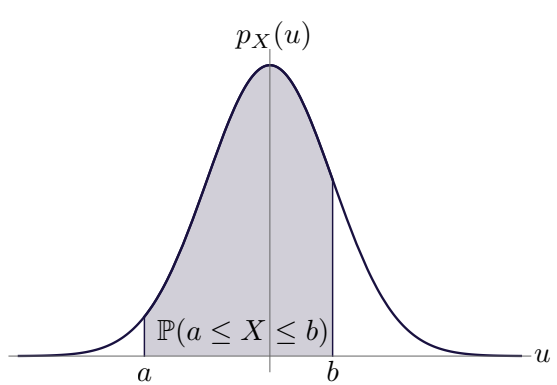

Figure 2.2: Probability of finding $X$ in the interval $[a, b]$.

2. Limiting values: $\lim _{x \rightarrow-\infty} P_{X}(x)=0$, and $\lim _{x \rightarrow+\infty} P_{X}(x)=1$.

3. Right-continuity: $\lim _{\epsilon \searrow^{0}} P_{X}(x+\epsilon)=P_{X}(x), \quad \forall x \in \mathbb{R}$.

Though it no longer makes sense to talk about probabilities of individual values of $x$, we can calculate from (2-2) the probabilities of $X$ falling within intervals as

$$
\mathrm{P}(a \leq X \leq b)=P_{X}(b)-P_{X}(a) .
$$

If the distribution function $P$ is differentiable, it is also useful to define the probability density function (pdf) $p_{X}: \mathbb{R} \rightarrow[0, \infty)$ of $X \mathrm{as}^{2}$

$$
p_{X}(x):=\frac{\mathrm{d}}{\mathrm{d} x} P_{X}(x)
$$

and hence, (see Fig. 2.1)

$$
P_{X}(x)=\int_{-\infty}^{x} \mathrm{~d} u p_{X}(u)
$$

Since $\lim _{x \rightarrow+\infty} P_{X}(x)=1$, the pdf must satisfy

$$
\int_{-\infty}^{\infty} \mathrm{d} u p_{X}(u)=1
$$

Furthermore,

$$
\mathbb{P}(a \leq X \leq b)=\int_{a}^{b} \mathrm{~d} u p_{X}(u),
$$

that is, the probability that a random variable $\mathrm{X}$ has value between $x=a$ and $x=b$ is the area under the graph of the density function between $x=a$

${ }^{2}$ In statistical physics, a non-formal reformulation of the relation (2-3) between the derivative of the cdf and the pdf is generally used as the definition of the probability density function: If $\mathrm{d} t$ is an infinitely small number, the probability that $X$ is included within the interval $[t, t+\mathrm{d} t]$ is equal to $p_{X}(t) \mathrm{d} t$, or: $\mathbb{P}(t \leq X \leq t+\mathrm{d} t) \approx p_{X}(t) \mathrm{d} t$. 
and $x=b$ (see Fig. 2.2).

It is often convenient to summarize a probability distribution by one or two statistics that characterize its main features. The most common are the expectation or mean, and variance. In the case of a continuous random variable $X$ with probability density function $p_{X}$, the expectation is

$$
\mu \equiv \mathbb{E}[X]:=\int_{-\infty}^{\infty} \mathrm{d} x p_{X}(x) x
$$

More generally, the $k^{t h}$ moment is defined for $k \in \mathbb{N}$ by

$$
m_{k}:=\mathbb{E}\left[X^{k}\right]=\int_{-\infty}^{\infty} \mathrm{d} x p_{X}(x) x^{k}
$$

The variance of a random variable $X$ is the expected value of the squared deviation from the mean of $X$, i.e.,

$$
\sigma^{2} \equiv \operatorname{Var}(X):=\int_{-\infty}^{\infty} \mathrm{d} x p_{X}(x)(x-\mu)^{2}=\mathbb{E}\left[X^{2}\right]-\mathbb{E}^{2}[X]
$$

\subsection{1}

\section{Special Probability Distributions}

In both discrete and continuous cases there are some well-known families of probability distributions. An important example is the Poisson distribution [18], corresponding to a random variable having probability mass function

$$
p(x ; \lambda)=\mathbb{P}(X=x)=\frac{e^{-\lambda} \lambda^{x}}{x !}, \quad x \in \mathbb{N}_{0},
$$

where $\lambda>0$. The Poisson distribution is used as a model for the occurrence of randomly occurring events in time: if events occur randomly in time at an average rate of $\lambda$, and independently of the time since the last event, then the number of events arising in a unit time interval, $X$, has a Poisson distribution with parameter $\lambda$, denoted as $X \stackrel{d}{=} \operatorname{Pois}(\lambda)$.

Perhaps the most widely used distribution in the continuous case is the normal distribution. A random variable $X$ is said to have a normal distribution with parameters $\mu$ and $\sigma$, denoted $X \stackrel{d}{=} \mathrm{N}\left(\mu, \sigma^{2}\right)$, if its probability density function has the form 


$$
p(x ; \mu, \sigma)=\frac{1}{\sqrt{2 \pi \sigma^{2}}} \exp \left(-\frac{(x-\mu)^{2}}{2 \sigma^{2}}\right), \quad-\infty<x<\infty
$$

where $\mu=\mathbb{E}[X]$ and $\sigma^{2}=\operatorname{Var}(X)$ are fixed parameters. The corresponding cdf of (2-12) can be expressed in terms of the error function:

$$
P(x ; \mu, \sigma)=\frac{1}{2}\left[1+\operatorname{erf}\left(\frac{x-\mu}{\sqrt{2} \sigma}\right)\right], \quad-\infty<x<\infty .
$$

In the case $X$ has normal distribution $\mathrm{N}\left(\mu, \sigma^{2}\right)$, it can easily be shown that

$$
Z:=\frac{X-\mu}{\sigma} \stackrel{d}{=} \mathrm{N}(0,1)
$$

The variable $Z$ is said to have standard normal distribution .

Another example in the continuous case is the exponential distribution which is the probability distribution that describes the time between events in a Poisson process, i.e., a process in which events occur continuously and independently at a constant average rate and it has the key property of being memoryless [17]. The exponential distribution is an appropriate model if the following conditions are true:

- $X$ is the time (or distance) between events, with $X>0$.

- The occurrence of one event does not affect the probability that a second event will occur. That is, events occur independently.

- The rate at which events occur, $\lambda$, is constant.

- Two events can not occur at exactly the same instant.

A random variable $X$ is said to have exponential distribution with constant rate parameter $\lambda>0$, denoted $X \stackrel{d}{=} \operatorname{Exp}(\lambda)$, if its pdf has the form

$$
p(x ; \lambda)= \begin{cases}0, & x<0 \\ \lambda e^{-\lambda x}, & x \geq 0\end{cases}
$$

Hence, Its cdf is

$$
P(x ; \lambda)= \begin{cases}0, & x<0 \\ 1-e^{-\lambda x}, & x \geq 0 .\end{cases}
$$

Next important example in the continuous case is Pareto or power law distribution with pdf 


$$
p\left(x ; x_{m}, \alpha\right)= \begin{cases}0, & x<x_{m}, \\ \frac{\alpha x_{m}^{\alpha}}{x^{\alpha+1}}, & x \geq x_{m},\end{cases}
$$

where the scale parameter $x_{m}>0$ is the minimum possible value of $X \stackrel{d}{=}$ Pareto $\left(x_{m}, \alpha\right)$, and the shape parameter or tail index $\alpha>0$ determines the strength of the tail at large $x$ (see Fig. 2.4). The corresponding cdf

$$
P\left(x ; x_{m}, \alpha\right)= \begin{cases}0, & x<x_{m}, \\ 1-\left(\frac{x_{m}}{x}\right)^{\alpha}, & x \geq x_{m},\end{cases}
$$

is not used frequently but rather its complement, $1-P(x)=: \bar{P}(x)$ (see Sec. $2.4)$, as it is easier to normalize and compare it to the data: the ordinate simply specifies the number of data points (measurements, events) that were larger than the chosen value on the abscissa.

\subsection{2}

\section{Moment-Generating Functions}

Although the mean and variance of a random variable contain important information about the random variable, they do not contain all the available information about the density function of a random variable.

An alternative approach to specify the probability distribution of a random variable $X$ is based on its moment-generating function which encapsulates all the information about the random variable, or more precisely, about its pdf. The moment-generating function $M_{X}: \mathbb{R} \rightarrow[0, \infty]$ associated with a random variable $X$ is defined by

$$
M_{X}(t):=\mathbb{E}\left[e^{t X}\right], \quad t \in \mathbb{R},
$$

wherever this expectation exists, i.e., the domain in which $M_{X}$ defined is $D_{X}=\left\{t: M_{X}(t)<\infty\right\}$. In other terms, the moment-generating function can be interpreted as the expectation of the random variable $e^{t X}$. If $X$ is a continuous random variable with pdf $p_{X}$, then

We note that

$$
M_{X}(t)=\int_{-\infty}^{\infty} \mathrm{d} x e^{t x} p_{X}(x)
$$

$$
M_{X}(0)=\int_{-\infty}^{\infty} \mathrm{d} x p_{X}(x)=1,
$$

which means we always have $t=0 \in D_{X}$. As an example, if $X \stackrel{d}{=} \operatorname{Exp}(\lambda)$, then 


$$
M_{X}(t)=\int_{0}^{\infty} \mathrm{d} x e^{t x} \lambda e^{-\lambda x}=\frac{\lambda}{\lambda-t}, \quad t<\lambda,
$$

and if $X \stackrel{d}{=} \mathrm{N}(0,1)$, we have

$$
\begin{aligned}
M_{X}(t) & =\int_{-\infty}^{\infty} \mathrm{d} x e^{t x} \frac{1}{\sqrt{2 \pi}} e^{-x^{2} / 2} \\
& =\frac{1}{\sqrt{2 \pi}} e^{t^{2} / 2} \int_{-\infty}^{\infty} \mathrm{d} x e^{-(x-t)^{2} / 2} \\
& =e^{t^{2} / 2}, \quad-\infty<t<\infty
\end{aligned}
$$

If $X \stackrel{d}{=} \mathrm{N}\left(\mu, \sigma^{2}\right)$, by a straightforward calculation, we find that $\mathrm{X}$ has the moment generating function

$$
M_{X}(t)=e^{\mu t+\sigma^{2} t^{2} / 2}, \quad(-\infty<t<\infty) .
$$

Moment generating functions provide many advantages. Here, we name a few:

- The moment-generating function of a random variable $X$ uniquely determines its pdf $p_{X}$. More precisely, let $M_{X}(t)$ and $M_{Y}(t)$ denotes the momentgenerating functions of random variables $X$ and $Y$, respectively. If both moment-generating functions exist and $M_{X}(t)=M_{Y}(t)$, for all values of $t$, then $X$ and $Y$ have the same probability distribution, $p_{X}(x)=p_{Y}(x)$.

- They provide an easy way of calculating the moments of a distribution. To see this, we notice that

$$
M_{X}(t)=\mathbb{E}\left[e^{t X}\right]=\mathbb{E}\left[\sum_{k=0}^{\infty} \frac{(t X)^{k}}{k !}\right]=\sum_{k=0}^{\infty} \frac{t^{k} \mathbb{E}\left[X^{k}\right]}{k !}=\sum_{k=0}^{\infty} \frac{t^{k} m_{k}}{k !} .
$$

We thus have

$$
m_{k}=\left.\frac{\mathrm{d}^{k}}{\mathrm{~d} t^{k}} M_{X}(t)\right|_{t=0}
$$

For example, the mean $\mu$ and the variance $\sigma^{2}$ are

$$
\begin{aligned}
\mu & =M_{X}^{\prime}(0) \\
\sigma^{2} & =M_{X}^{\prime \prime}(0)-\left(M_{X}^{\prime}(0)\right)^{2} .
\end{aligned}
$$

- They provide an easy way of characterizing the distribution of the sum of independent random variables. Strictly speaking, assume $S_{n}:=X_{1}+\cdots+X_{n}$ 
denote the sum of $n$ independent random variables $X_{i}$. From multiplicativity of expectation for independent random variables

$$
\mathbb{E}\left[e^{t S_{n}}\right]=\left[e^{t X_{1}} \cdots e^{t X_{n}}\right]=\mathbb{E}\left[e^{t X_{1}}\right] \cdots \mathbb{E}\left[e^{t X_{n}}\right]
$$

follows that

$$
M_{S_{n}}(t)=M_{X_{1}}(t) \cdots M_{X_{n}}(t) .
$$

If $X_{i}$ 's are identically distributed as $X$, then

$$
M_{S_{n}}(t)=\left(M_{X}(t)\right)^{n} .
$$

- They provide a bridge between complex analysis and probability, so that methods of complex analysis can be applied to probability problems (see Sec. $5.2 .1)$.

- They provide powerful tools for proving limit theorems, such as law of large numbers and the central limit theorem (see Sec. 2.3).

\section{2}

\section{Multivariate Random Variables}

A multivariate random variable is a vector of random variables

$$
X:=\left(X_{1}, \ldots, X_{k}\right)
$$

Each of the components $X_{i}$ is a random variable in its own right, but specification of the properties of $\boldsymbol{X}$ as a whole, requires information about the influence of every variable on each of the others.

Generalizing the single variable case, the joint cumulative distribution function of $\boldsymbol{X}$ is defined by

$$
P_{X}\left(x_{1}, \ldots, x_{n}\right):=\mathbb{P}\left(X_{1} \leq x_{1}, \ldots, X_{k} \leq x_{k}\right) .
$$

When $X_{i}$ are continuous random variables, and provided it exists, the joint probability density function (jpdf) is given by

$$
p_{\boldsymbol{X}}(\boldsymbol{x}):=\frac{\partial^{k}}{\partial x_{1} \cdots \partial x_{k}} P_{\boldsymbol{X}}(\boldsymbol{x})
$$

where $\boldsymbol{x} \equiv\left(x_{1}, \ldots, x_{k}\right)$. In this case

$$
P_{\boldsymbol{X}}(\boldsymbol{x})=\int_{-\infty}^{x_{1}} \cdots \int_{-\infty}^{x_{k}} \mathrm{~d} u_{k} \cdots \mathrm{d} u_{1} p_{\boldsymbol{X}}\left(u_{1}, \ldots, u_{k}\right)
$$

while for any set $\mathcal{A} \subset \mathbb{R}^{k}$, 


$$
\mathbb{P}(X \in \mathcal{A})=\int \cdots \int_{\mathcal{A}} \mathrm{d} \boldsymbol{u} p_{\boldsymbol{X}}(u)
$$

The pdf of each of the individual $X_{i}$, called marginal density function, is obtained by integrating out the other components. For instance,

$$
p_{X_{1}}\left(x_{1}\right)=\int_{-\infty}^{\infty} \cdots \int_{-\infty}^{\infty} \mathrm{d} u_{k} \cdots \mathrm{d} u_{2} p_{X}\left(x_{1}, u_{2}, \ldots, u_{k}\right)
$$

is the marginal probability density function of the component $X_{1}$. In the special situation where the outcome of one random variable has no effect on the probability distribution of another, the variables are said to be independent. Formally, the variables $X_{1}$ and $X_{2}$ are independent, if their jpdf factorizes, i.e.,

$$
p_{X_{1}, X_{2}}\left(x_{1}, x_{2}\right)=p_{X_{1}}\left(x_{1}\right) p_{X_{2}}\left(x_{2}\right) .
$$

Generally, an arbitrary set of random variables $X_{1}, \ldots, X_{k}$ are mutually independent if

$$
p_{X_{1}, \ldots, X_{k}}\left(x_{1}, \ldots, x_{k}\right)=\prod_{i=1}^{k} p_{X_{i}}\left(x_{i}\right)
$$

\section{3}

\section{Convergence of Sequence of Random Variables}

It is often difficult to perform exact calculations with probability distributions. This might be because the distribution is unknown, or simply because the analytical or computational burden is high. In these situations, it may be possible to approximate the true distribution by a simpler distribution obtained by a limiting process. This requires a definition of convergence of sequences of random variables to some limit random variable, which is an important concept in probability theory. There exist several different notions of convergence of random variables. Here, we introduce two of these notions, which in turn, we use them to prove two basic theorems of probability theory with wide range applications in statistics.

\subsection{1}

\section{Convergence in Probability and Weak Law of Large Numbers}

The first notion of convergence we introduce here which is used very often in statistics, is convergence in probability or weak convergence. The basic idea behind this type of convergence is that the probability of an "unusual" 
outcome becomes smaller and smaller as the sequence progresses. A sequence of random variables $X_{1}, X_{2}, \ldots$ converges in probability to a random variable $X$, denoted as

$$
X_{n} \stackrel{P}{\rightarrow} X
$$

if

$$
\lim _{n \rightarrow \infty} P\left(\left|X_{n}-X\right| \geq \epsilon\right)=0, \quad \forall \epsilon>0
$$

Convergence in probability can be used to prove the weak law of large numbers. Suppose $X_{1}, X_{2}, \ldots$ is a sequence of random variables with $\mathbb{E}\left[X_{1}\right]=$ $\mathbb{E}\left[X_{2}\right]=\cdots=\mu$. Then, weak law of large numbers states that

$$
\bar{X}_{n} \stackrel{P}{\rightarrow} \mu, \quad \text { as } n \rightarrow \infty .
$$

To prove this theorem using moment-generating functions, we have to rely on a theorem according which convergence of moment-generating functions implies weak convergence of distributions [20]. Then suppose $X_{1}, X_{2}, \ldots$ are sequence of iid random variables ${ }^{3}$ with well-defined moment-generating functions, and distributed as $X$ with mean $\mu$, therefore,

$$
\begin{aligned}
M_{\bar{X}_{n}}(t) & =\mathbb{E}\left[e^{t \bar{X}_{n}}\right] \\
& =\mathbb{E}\left[e^{\frac{t}{n}\left(X_{1}+\cdots+X_{n}\right)}\right] \\
& =\mathbb{E}\left[e^{\frac{t}{n} X_{1}}\right] \cdots \mathbb{E}\left[e^{\frac{t}{n} X_{n}}\right] \\
& =\left(\mathbb{E}\left[e^{\frac{t}{n} X}\right]\right)^{n} \\
& =\left(M_{X}\left(\frac{t}{n}\right)\right)^{n} \\
& =\left(M_{X}(0)+\frac{t}{n} M_{X}^{\prime}(0)+o\left(\frac{t}{n}\right)\right)^{n} \\
& \approx\left(1+\frac{t}{n} \mu\right)^{n}
\end{aligned}
$$

Therefore, $\lim _{n \rightarrow \infty} M_{\bar{X}_{n}}(t)=e^{t \mu}$. This means that the moment-generating function of the variable $\bar{X}_{n}$ converges to he moment-generating function a random variable with mean $\mu$, which in turn, means $\bar{X}_{n}$ converges in

${ }^{3}$ In probability theory and statistics, a sequence or other collection of random variables is independent and identically distributed (iid), if each random variable has the same probability distribution as the others and all are mutually independent. 
probability to $\mu$, as stated before.

By the law of large numbers, the empirical sample average $\bar{X}_{n}$ of the results obtained from a large number of trials, converges to the expected value $\mu$. Another theorem, i.e., classical central limit theorem, describes the size and the distributional form of the stochastic fluctuations around the deterministic number $\mu$ during this convergence.

\subsection{2}

\section{Convergence in Distribution and Central Limit Theorem}

The other notion of convergence which is useful for our purposes, is convergence in distribution. A sequence of random variables $X_{1}, X_{2}, \ldots$ having cumulative distribution functions $P_{1}, P_{2}, \ldots$, respectively, is said to converge in distribution to the random variable $X$, having distribution function $P$, denoted as

$$
X_{n} \rightsquigarrow X,
$$

if the cdf of $X_{n}$ converges pointwise to the cdf of $X$, i.e.,

$$
\lim _{n \rightarrow \infty} P_{n}(x)=P(x)
$$

for all points $x$ at which $P$ is continuous [21]. ${ }^{4}$ For statistical applications, the utility of establishing a limit distribution $P$ for a sequence of random variables $X_{1}, X_{2}, \ldots$ is usually to justify the use of $P$ as an approximation to the distribution of $X_{n}$ for large $n$.

The second fundamental result in probability theory, after the law of large numbers is central limit theorem, stated here in its simplest form: Let $S_{n}=X_{1}+\cdots+X_{n}$, where $X_{1}, X_{2}, \ldots$ are sequence of iid random variables, and distributed as $X$ with mean $\mu$ and finite variance $\sigma^{2}$. Then defining

$$
Z_{n}:=\frac{S_{n}-n \mu}{\sqrt{n \sigma^{2}}}
$$

we have

$$
Z_{n} \rightsquigarrow Z,
$$

${ }^{4}$ It is worth emphasizing that since convergence in distribution only considers the cdf, is in fact weaker than convergence in probability. 
as $n \rightarrow \infty$, where $Z$ is a standard normal random variable, i.e., $Z \stackrel{d}{=} \mathrm{N}(0,1)$. Here, we give a proof of this theorem, in the special case when the momentgenerating function of $X$ is finite: Let $Y:=\frac{X-\mu}{\sigma}$ and $Y_{i}:=\frac{X_{i}-\mu}{\sigma}$. Then $Y_{i}$ 's are independent and are distributed as $Y$, i.e., $\mathbb{E}\left[Y_{i}\right]=0$ and $\operatorname{Var}\left(Y_{i}\right)=1$. We can write then $Z_{n}=\left(Y_{1}+\cdots+Y_{n}\right) / \sqrt{n}$. Hence, ${ }^{5}$

$$
\begin{aligned}
M_{Z_{n}}(t) & =\mathbb{E}\left[e^{t Z_{n}}\right] \\
& =\mathbb{E}\left[e^{\frac{t}{\sqrt{n}} Y_{1}+\cdots+\frac{t}{\sqrt{n}} Y_{n}}\right] \\
& =\mathbb{E}\left[e^{\frac{t}{\sqrt{n}} Y_{1}}\right] \cdots \mathbb{E}\left[e^{\frac{t}{\sqrt{n}} Y_{n}}\right] \\
& =\left(\mathbb{E}\left[e^{\frac{t}{\sqrt{n}} Y}\right]\right)^{n} \\
& =\left(M_{Y}\left(\frac{t}{\sqrt{n}}\right)\right)^{n} \\
& =\left(M_{Y}(0)+\frac{t}{\sqrt{n}} M_{Y}^{\prime}(0)+\frac{t^{2}}{n} M_{Y}^{\prime \prime}(0)+o\left(\frac{t^{2}}{n}\right)\right)^{n} \\
& \approx\left(1+\frac{t^{2}}{2 n}\right)^{n}
\end{aligned}
$$

Therefore, $\lim _{n \rightarrow \infty} M_{Z_{n}}(t)=e^{t^{2} / 2}=M_{Z}(t)$, where $Z \stackrel{d}{=} \mathrm{N}(0,1)$ (see (2-23)). Then, the convergence in distribution $Z_{n} \leadsto Z$ can be deduced by the convergence of the moment-generating functions. The central limit theorem thus states that there exist sequences of numbers $\left\{a_{n}>0\right\}=\{\sigma \sqrt{n}\}$ and $\left\{b_{n}\right\}=\{\mu n\}$, such that $\frac{S_{n}-b_{n}}{a_{n}}$ converges to a standard normal distribution, regardless of the underlying distribution of the $X_{i}$. This is manifestation of a general phenomenon known as universality. Analogous arguments are used in next chapter to obtain approximating distributions for sample extremes.

Finally, we notice that central limit theorem is a stronger result than the weak law of large numbers: it gives more details about what the asymptotic distribution actually looks like. In fact, we know not only that (from the law of large numbers) the distribution of the sample mean approaches the degenerate distribution on $\mathbb{E}[X]$, but moreover (from the central limit theorem) we know exactly what this distribution looks like asymptotically,

${ }^{5}$ We notice that according to (2-42), the law of large numbers corresponds to a first order expansion of the moment-generating function, while according to (2-47), the central limit theorem corresponds to its second order expansion. 
if we zoom in on $\mathbb{E}[X]$, to a scale of $N^{-1 / 2}$.

\section{4}

\section{Tail Distribution}

Sometimes, it is useful to study the opposite question and ask how often the random variable is above a particular level. This is called the complementary cumulative distribution function or simply the tail distribution, and is defined $a s^{6}$

$$
\begin{aligned}
\bar{P}_{X}(x) & :=\mathbb{P}(X>x) \\
& =\int_{x}^{\infty} \mathrm{d} u p_{X}(u) \\
& =\int_{-\infty}^{\infty} \mathrm{d} u p_{X}(u)-\int_{-\infty}^{x} \mathrm{~d} u p_{X}(u),
\end{aligned}
$$

which using (2-5) and (2-6) gives

$$
\bar{P}_{X}(x)=1-P_{X}(x) .
$$

\subsection{1}

\section{Concentration Inequalities and Tail Bounds}

In probability theory, concentration inequalities provide bounds on how a random variable deviates from some value (typically, its expected value). For instance, we saw that the laws of large numbers state that sums of independent random variables are close to their expectation with a large probability. Such sums are the most basic examples of random variables concentrated around their mean. Concentration inequalities can be classified according to how much information about the random variable is needed in order to use them. One way to control the tail distribution $\bar{P}_{X}(x)$ is by controlling the moments of the random variable $X$. Gaining control of higher-order moments leads to correspondingly sharper bounds on tail probabilities, ranging from Markov's inequality (which requires only existence of the first moment) to the Chernoff bound (which requires existence of the moment generating function).

\footnotetext{
${ }^{6}$ In survival analysis, $\bar{P}_{X}(x)$, or simply $\bar{P}(x)$, is called the survival function and denoted $S(x)$, while the term reliability function is common in engineering.
} 
The most elementary tail bound is Markov's inequality which does not need any knowledge of the distribution of the non-negative random variable $X$, except that it has a finite mean. It states that [22]

$$
\bar{P}_{X}(x) \leq \frac{\mathbb{E}[X]}{x}, \quad \forall x>0 .
$$

Markov's inequality can be applied to a random variable $Y=f(X)$, in which $f: X \rightarrow \mathbb{R}^{+}$is a strictly increasing and non-negative function of $X$ :

$$
\bar{P}_{X}(x)=\mathbb{P}(X \geq x)=\mathbb{P}(f(X) \geq f(x)) \leq \frac{\mathbb{E}[f(X)]}{f(x)} .
$$

With a suitable choice for the function $f$, we can obtain better bounds. For example, if we pick $f(X)=X^{2}$ for a random variable $X$ that also has a finite variance, then we obtain a tighter bound [22]:

$$
\mathbb{P}(|X-\mathbb{E}[X]| \geq x)=\mathbb{P}\left((X-\mathbb{E}[X])^{2} \geq x^{2}\right) \leq \frac{\mathbb{E}\left[(X-\mathbb{E}[X])^{2}\right]}{x^{2}}
$$

which means

$$
\mathbb{P}(|X-\mathbb{E}[X]| \geq x) \leq \frac{\operatorname{Var}(X)}{x^{2}}, \quad \forall x>0 .
$$

or equivalently

$$
\mathbb{P}(|X-\mathbb{E}[X]| \geq x \operatorname{Var}(X)) \leq \frac{1}{x^{2}}, \quad \forall x>0 .
$$

This is called Chebyshev inequality. Hence, Chebyshev's inequality follows by applying Markov's inequality to the non-negative random variable $Y:=(X-\mathbb{E}[X])^{2}$ and is a simple form of concentration inequality, guaranteeing that $X$ is close to its mean $\mathbb{E}[X]$, whenever its variance is small.

Markov's inequality can be applied to random variables with higher order moments. For instance, whenever $X$ has a moment of order $k$, an application of Markov's inequality to the random variable $|X-\mathbb{E}[X]|^{k}$ yields

$$
\mathbb{P}\left(|X-\mathbb{E}[X]|^{k} \geq x\right) \leq \frac{\mathbb{E}\left[|X-\mathbb{E}[X]|^{k}\right]}{x^{k}}, \quad(\forall x>0)
$$

The same procedure can be applied to functions other than polynomials $|X-\mathbb{E}[X]|^{k}$. For instance, suppose that the random variable $X$ has a moment generating function in a neighborhood of zero, meaning that there is some constant $t_{0}>0$ such that the function $M_{X-\mathbb{E}[X]}(t)=\mathbb{E}\left[e^{t(X-\mathbb{E}[X])}\right]$ exists for all $0 \leq t \leq t_{0}$. In this case, we may apply Markov's inequality to the random variable $Y:=e^{t(X-\mathbb{E}[X])}$, thereby obtaining the upper bound 


$$
\mathbb{P}((X-\mathbb{E}[X]) \geq x)=\mathbb{P}\left(e^{t(X-\mathbb{E}[X])} \geq e^{t x}\right) \leq e^{-t x} M_{X-\mathbb{E}[X]}(t) .
$$

Optimizing our choice of $t$ so as to obtain the tightest result, yields the Chernoff bound [23]:

$$
\bar{P}_{X-\mathbb{E}[X]}(x) \leq \inf _{t \in\left[0, t_{0}\right]}\left\{e^{-t x} M_{X-\mathbb{E}[X]}(t)\right\} .
$$

This result is usually stated in logarithmic form. To do this, let's define $\Lambda(t):=\log \left(M_{X-\mathbb{E}[X]}(t)\right)$. Then by taking logarithm of both side of (2-57), we get

$$
\log \left(\bar{P}_{X-\mathbb{E}[X]}(x)\right) \leq-\Lambda^{*}(x)
$$

in which

$$
\Lambda^{*}(x):=\sup _{t \in\left[0, t_{0}\right]}\{x t-\Lambda(t)\}
$$

is the Legendre-Fenchel transformation of $\Lambda(t)[22,24,25]$ (see Fig. 2.3).

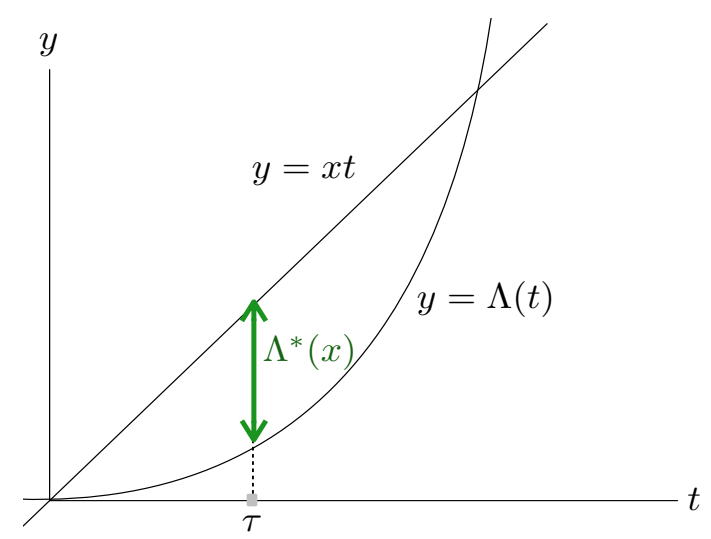

Figure 2.3: The function $\Lambda(t)$ plotted against the line $y=x t$, in the case when $\Lambda^{\prime}(0)=0$. The point $\tau$ marks the value of $t$ at which $x t-\Lambda(t)$ is a maximum, and this maximum is denoted $\Lambda^{*}(x)$.

Chernoff inequality gives exponentially decreasing bounds on tail distributions and hence, is a sharper bound than the known first or second moment based tail bounds, i.e., Markov's inequality or Chebyshev inequality, respectively, which only yield power-law bounds on tail decay. Moreover, the Chernoff bound is most widely used in practice, due to the ease of manipulating moment generating functions. As an example if $X \stackrel{d}{=} \mathrm{N}\left(\mu, \sigma^{2}\right)$, we have (see $(2-24))$ 


$$
\sup _{-\infty<t<\infty}\left\{t x-\log \left(M_{X}(t)\right)\right\}=\sup _{-\infty<t<\infty}\left\{t x-\frac{t^{2} \sigma^{2}}{2}\right\}=\frac{x^{2}}{2 \sigma^{2}}
$$

From the Chernoff bound (2-58), we conclude that any random variable $X \stackrel{d}{=} \mathrm{N}\left(\mu, \sigma^{2}\right)$ satisfies

$$
\bar{P}_{X}(\mu+x) \leq e^{-\frac{x^{2}}{2 \sigma^{2}}}, \quad \forall x \geq 0
$$

which is tight up to polynomial factor corrections. ${ }^{7}$

\subsection{2}

\section{Classification of tails}

Generally, probability distributions are classified by comparing their tail distribution with the exponential tail distribution. In the case of exponential distribution with pdf given in (2-15), the tail distribution is

$$
\bar{P}(x ; \lambda)= \begin{cases}0, & x<0, \\ e^{-\lambda x}, & x \geq 0 .\end{cases}
$$

Then, a distribution $P$ is called heavy-tailed iff its tail function $\bar{P}$ fails to be bounded by any exponentially decreasing function [26, 27], i.e.,

$$
\lim _{x \rightarrow \infty} e^{\lambda x} \bar{P}(x)=\infty, \quad \forall \lambda>0 .
$$

In other words, heavy-tailed distributions have heavier tails than the exponential distribution. This implies that large values can occur in a sample with non-negligible probability. The definition (2-63) is equivalent to the statement that the moment generating function of $P, M(t)$, does not exist for $t>0$ [26]; that is, a distribution $P$ with pdf $p$ is heavy-tailed iff:

$$
M(t)=\int_{-\infty}^{\infty} \mathrm{d} x e^{t x} p(x)=\infty, \quad \forall t>0
$$

An important subclass of heavy-tailed class are fat-tailed distributions which have power law decay in the tail of the distribution. ${ }^{8}$ The distribution of a random variable $X$ is said to have a fat tail with tail index $\alpha$, if its asymptotic behaviour is given by

${ }^{7}$ Let $p_{X}(x)=\frac{1}{\sqrt{2 \pi}} e^{-x^{2} / 2}$ be the pdf of a standard normal variable $X \stackrel{d}{=} \mathrm{N}(0,1)$ (see (2-12)), then the following two-sided inequality holds:

$$
p_{X}(x)\left(\frac{1}{x}-\frac{1}{x^{3}}\right) \leq \bar{P}_{X}(x) \leq p_{X}(x)\left(\frac{1}{x}-\frac{1}{x^{3}}+\frac{3}{x^{5}}\right) .
$$

${ }^{8}$ However, they generally do not follow a power law everywhere. 


$$
\bar{P}(x) \underset{x \rightarrow \infty}{\sim} x^{-\alpha}, \quad \alpha>0,
$$

that is, if $X$ has pdf

$$
p(x) \underset{x \rightarrow \infty}{\sim} x^{-(1+\alpha)}, \quad \alpha>0 .
$$

If we are "sufficiently close" to infinity to estimate the tail indices of two distributions, then we can meaningfully compare their tail heaviness by comparing their tail indices. (see Fig. 2.4).

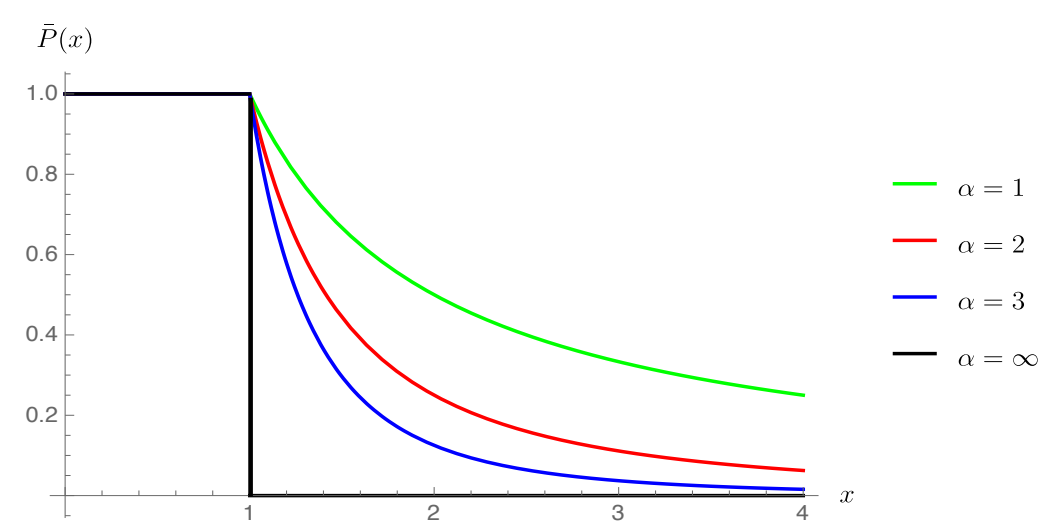

Figure 2.4: Tail of Pareto distribution with $x_{m}=1$, and for different values of tail index $\alpha$.

An example of fat-tailed distributions is Pareto distribution with cdf given in (2-18). Its tail is thus:

$$
\bar{P}\left(x ; x_{m}, \alpha\right)= \begin{cases}1, & x<x_{m} \\ \left(\frac{x_{m}}{x}\right)^{\alpha}, & x \geq x_{m}\end{cases}
$$

As we already expect, its moment generating function

$$
M\left(t ; x_{m}, \alpha\right)=\alpha\left(-x_{m} t\right)^{-\alpha} \Gamma\left(-\alpha,-x_{m} t\right)
$$

is only defined for $t \leq 0$, where $\Gamma(a, x)$ is the upper incomplete gamma function (see App. B). Moreover, all moments $m_{k}$ are infinite for $k \geq \alpha$.

Power laws usually occur in perturbed form. Deviation from power law distributions leads to another class of heavy-tailed distributions, i.e., distributions with regularly varying tails. A random variable $X$ is said to have a regularly varying tail distribution $\bar{P}$ with index $\alpha>0$ if $\bar{P}$ is a regularly varying function, with index $-\alpha, \bar{P} \in \mathcal{R} \mathcal{V}_{\infty}(-\alpha)$ (see App. A), i.e., 


$$
\bar{P}(x)=x^{-\alpha} \Psi(x),
$$

in which $\Psi(x)$ is a slowly varying function. This can be reformulated solely in terms of $\bar{P}$ (see App. A)

$$
\frac{\bar{P}(y x)}{\bar{P}(x)} \underset{x \rightarrow \infty}{\sim} y^{-\alpha}, \quad \forall y>0 .
$$




\title{
Asymptotic Models of Classical Extreme Value Theory
}

The sole aim of science is the honor of human mind, and from this point of view, a question about numbers is as important as a question about the system of the world.

\author{
C. G. J. Jacobi
}

This chapter is concerned with the central result of classical extreme value theory, the extermal types theorem, which specifies the possible forms for the limiting distribution of maxima (minima) in sequence of iid random variables. First, we briefly discuss the asymptotic formulation of classical extreme value theory (EVT), and then introduce the three classes of extreme value distributions along with their tail behaviour introduced in the previous chapter. Finally, necessary and sufficient condition for the extermal types theorem are provided in terms of the concept of domain of attraction.

\section{1}

\section{Model Formulation}

The asymptotic theory of sample extremes has been developed in parallel with the central limit theory, and in fact the two theories have some resemblance. Let $X_{1}, \ldots, X_{n}$ be a sample of $n$ independent and identically distributed random variables with common cdf $P$. The sample data are typically used to study properties about the distribution function

$$
P(x)=\mathbb{P}(X \leq x) .
$$

In classical statistics, one is often interested in the behaviour of the mean or average. This average will then be described through the expected value $\mathbb{E}(X)$ of the distribution. On the basis of the law of large numbers, the sample mean $\bar{X}$ is used as an estimation of $\mathbb{E}(X)$. Furthermore, the central limit theorem yields the asymptotic behaviour of the sample mean in case the sample size, $n$, is sufficiently large, a condition necessary when invoking 
the central limit theorem.

Asymptotic models of classical extreme value theory; however, are mainly concerned with the distribution of the random variable

$$
M_{n}:=\max \left\{X_{1}, \ldots, X_{n}\right\},
$$

when $n$ is large. In theory (under the assumption that $X_{i}$ 's are iid random variables, and the fact that if $M_{n}$ should be smaller than some $x$, then all $X_{i}$ simultaneously should be smaller than $x$ ) the distribution of $M_{n}$ can be derived exactly for all values of $n:^{1}$

$$
\begin{aligned}
\mathbb{P}\left(M_{n} \leq x\right) & =\mathbb{P}\left(X_{1} \leq x, \ldots, X_{n} \leq x\right) \\
& =\prod_{i=1}^{n} \mathbb{P}\left(X_{i} \leq x\right) \\
& =(P(x))^{n} .
\end{aligned}
$$

However, this is not of practical use, since the distribution function $P$ is not known a priori. A possible way is to use standard statistical techniques to estimate $P$ from observed data, but even small errors in $P$ (particularly in its tails) may imply large deviations in $P^{n}$. An alternative approach is to accept that $P$ is unknown, and look for approximate families for $P^{n}$ by which extreme data can be modeled directly [28, 29].

Moreover,

$$
\lim _{n \rightarrow \infty}(P(x))^{n}= \begin{cases}0, & x<x_{+}, \\ 1, & x=x_{+},\end{cases}
$$

in which $x_{+}$is the outer edge of the support of $P, x_{+} \equiv \sup \{x: P(x)<1\}$. Hence, the distribution of $M_{n}$ degenerates to a point at $x_{+}$. In analogy with central limit theorem for partial sums, the degeneracy of the limit can be avoided by allowing a linear rescaling ${ }^{2}$ of the variable $M_{n}$ :

${ }^{1}$ The corresponding pdf denoted $\pi(x)$ is

$$
\pi(x)=n(P(x))^{n-1} p(x)
$$

where $p(x)$ is the corresponding pdf of $P$.

${ }^{2}$ One can consider a wider class of resaclings. However, this linear rescaling already leads to a sufficiently rich theory [30]. 


$$
M_{n}^{*}:=\frac{M_{n}-b_{n}}{a_{n}}
$$

for sequences of constants $\left\{a_{n} \in \mathbb{R}^{+}\right\}$and $\left\{b_{n} \in \mathbb{R}\right\}$. Appropriate choices of the $a_{n}$ and $b_{n}$ stabilize the location and scale of $M_{n}^{*}$ as $n$ increases, avoiding the difficulties that arise with the variable $M_{n}$. We therefore seek limit distributions for $M_{n}^{*}$, with appropriate choices of $a_{n}$ and $b_{n}$, rather than $M_{n}$.

\section{2}

\section{The Three Types of Extreme Value Distributions}

Suppose there exist sequences of constants $\left\{a_{n} \in \mathbb{R}^{+}\right\}$and $\left\{b_{n} \in \mathbb{R}\right\}$ such that $\left(M_{n}-b_{n}\right) / a_{n}$ converges in distribution to a nontrivial limit, i.e.,

$$
\lim _{n \rightarrow \infty} \mathbb{P}\left(\frac{M_{n}-b_{n}}{a_{n}} \leq x\right) \equiv \lim _{n \rightarrow \infty}\left(P\left(a_{n} x+b_{n}\right)\right)^{n}=: G(x),
$$

for every continuity point $x$ of $G$, where $G$ is a non-degenerate distribution function. ${ }^{3}$

The problem is twofold: first, find all possible (non-degenerate) distribution functions $G$ that can occur as a limit in (3-6), called stable distributions or extreme value distributions; next, for each of those limit distributions, find necessary and sufficient conditions on the distribution $P$ for which there exist sequences $\left\{a_{n} \in \mathbb{R}^{+}\right\}$and $\left\{b_{n} \in \mathbb{R}\right\}$, such that (3-6) holds. The class of distributions $P$ satisfying (3-6), is called the domain of attraction of $G$ and we write $P \in \mathcal{D}(G)[31,32]$.

Surprisingly, there are only three types of limiting distributions. This fundamental result, known as Fisher-Tippett-Gnedenko theorem, was first discovered by Fisher and Tippett [33], and later discussed more completely by Gnedenko [34]. This theorem states that the maximum of a sample of iid random variables, ${ }^{4}$ after proper rescaling, can only converge in distribution to one of the following families $[28,30,35,36]$ :

\section{Gumbel distribution}

${ }^{3} \mathrm{~A}$ non-degenerate distribution function is a limiting distribution function that is not concentrated on a single point.

${ }^{4}$ In fact, under suitable conditions, the theorem holds for dependent random variables as well [35]. 


$$
G(x)=\exp \left\{-\exp \left[-\left(\frac{x-b}{a}\right)\right]\right\}, \quad-\infty<x<\infty .
$$

\section{Fréchet distribution}

$$
G(x)= \begin{cases}0, & x \leq b \\ \exp \left\{-\left(\frac{x-b}{a}\right)^{-\alpha}\right\}, & x>b\end{cases}
$$

III. Weibull distribution

$$
G(x)= \begin{cases}\exp \left\{-\left[-\left(\frac{x-b}{a}\right)^{\alpha}\right]\right\}, & x<b \\ 1, & x \geq b\end{cases}
$$

For all three types, $a>0$ and $b \in \mathbb{R}$ and, in the case of families II and III, $\alpha>0$. In other words, the rescaled sample maxima $\left(M_{n}-b_{n}\right) / a_{n}$ converge in distribution to a variable having a distribution within one of the families labeled I, II, and III. Collectively, these three classes of distribution are termed as extreme value distributions. Each family has a location parameter $b$ and a scale parameter $a$; additionally, the Fréchet and Weibull families have a shape parameter or tail index $\alpha$.

This implies that, when $M_{n}$ can be stabilized with suitable sequences $\left\{a_{n}\right\}$ and $\left\{b_{n}\right\}$, the corresponding normalized variable $M_{n}^{*}$ has a limiting distribution that must be one of the three types of extreme value distribution. The remarkable feature of this result is its universality; that is, the three types of extreme value distributions are the only possible limits for the distributions of the $M_{n}^{*}$, regardless of the distribution $P$. In this sense the Fisher-Tippett-Gnedenko theorem provides an extreme value analog of the central limit theorem (see the argument at the end of Sec. 2.3.2).

Although the distributions are known as extreme value, it must be borne in mind that they do not represent distributions of all kinds of extreme values (e.g., in samples of finite size), and they can be used empirically (without an asymptotic model), using treshold models [28, 36].

\section{3}

\section{The Generalized Extreme Value Distribution for Maxima}

The three types of limits already discussed, have distinct forms of behaviour, corresponding to the different forms of tail behaviour for the distribution 
function $P$ of the $X_{i}$. This can be made precise by considering the behaviour of the limit distribution $G$ at the outer edge of its support, $x_{+}$. For the Weibull distribution $x_{+}$is finite, while for both the Fréchet and Gumbel distributions $x_{+}=\infty$ (see Fig. 3.1); However, $G$ decays exponentially for the Gumbel distribution and polynomially for the Fréchet distribution, corresponding to different rates of decay in the tail of $P$. It follows that in applications, the three different families give quite different representations of extreme value behaviour. A way to apply extreme value theory, its to adopt one of the three families, and then to estimate the relevant parameters of that distribution. But there are two weaknesses: first, a technique is required to choose which of the three families is most appropriate for the given data; second, once the choice is made, subsequent inferences presume this choice to be correct, and do not allow for the uncertainty such a selection involves, even though this uncertainty may be substantial.

An alternative approach is to reformulate these three models in a single model. Indeed, it is straightforward to check that the Gumbel, Fréchet and Weibull families can be combined into a single family of models having distribution functions of the form

$$
G(x ; \mu, \sigma, \xi):=\exp \left\{-\left[1+\xi\left(\frac{x-\mu}{\sigma}\right)\right]^{-1 / \xi}\right\},
$$

defined on the set $\{x: 1+\xi(x-\mu) / \sigma>0\}$, where the parameters satisfy $-\infty<\mu<\infty, \sigma>0$ and $-\infty<\xi<\infty$. This is the generalized extreme value (GEV) family of distributions or Fisher-Tippett-Gnedenko distribution $[28,29]$. The model has three parameters: a location parameter $\mu$, a scale parameter $\sigma$, and a shape parameter $\xi$. Evidently the value of $\xi$ dictates the type of extreme value distribution, thus $\xi$ is also referred to as extreme value index.

Type II and type III classes of extreme value distribution correspond respectively to the cases $\xi>0$ and $\xi<0$ in this parameterization and the subset of the GEV family with $\xi=0$ is interpreted as the limit of (3-10) as $\xi \rightarrow 0$, leading to the Gumbel family with distribution function 


$$
\begin{aligned}
G(x ; \mu, \sigma, 0) & :=\lim _{\xi \rightarrow 0} G(x ; \mu, \sigma, \xi) \\
& =\exp \left\{-\exp \left[-\left(\frac{x-\mu}{\sigma}\right)\right]\right\},-\infty<x<\infty
\end{aligned}
$$

For type I $(\xi=0)$, the outer edge of the support of distribution tends to infinity. The distribution is, however, rather light-tailed: $1-G(x ; 0,1,0) \sim e^{-x}$ as $x \rightarrow \infty$, and all moment exist. For type II $(\xi>0)$, the outer edge of the support of distribution is infinity. Moreover, as $x \rightarrow \infty$, $1-G(x ; 0,1, \xi) \sim(\xi x)^{-1 / \xi}$, i.e., the distribution has a rather heavy right tail; for example, moments greater than or equal to $1 / \xi$ do not exist. Finally for type III $(\xi<0)$, the outer edge of the support of distribution is $-\xi^{-1}$, so it has a short tail, verifying $1-G\left(-\xi^{-1}-x ; 0,1, \xi\right) \sim(-\xi x)^{-1 / \xi}$ as $x \searrow 0$.

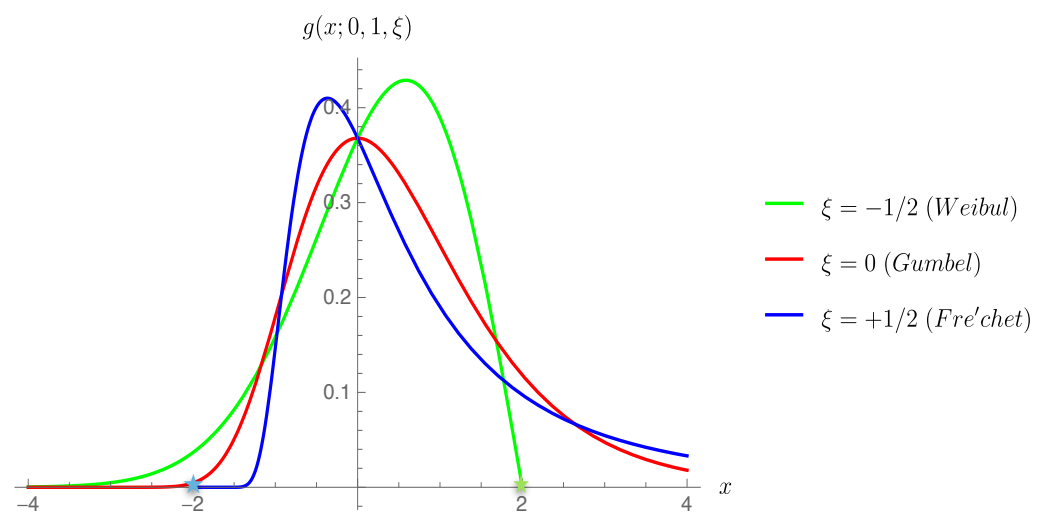

Figure 3.1: Generalized extreme value densities, all with $\mu=0$ and $\sigma=1$, and different extreme value index $\xi$. Asterisks mark endpoints of the supports.

The density function corresponding to (3-10) is (see Fig. 3.1)

$$
g(x ; \mu, \sigma, \xi)=\frac{1}{\sigma} \exp \left[-\left(\frac{x-\mu}{\sigma}\right)\right] \exp \left\{-\left[1+\xi\left(\frac{x-\mu}{\sigma}\right)\right]^{-1 / \xi}\right\},
$$

again defined on the set $\{x: 1+\xi(x-\mu) / \sigma>0\}$ and is zero outside of the relevant support. In the case $\xi=0$, the density is positive on the whole real line and is equal to

$$
g(x ; \mu, \sigma, 0)=\frac{1}{\sigma} \exp \left[-\left(\frac{x-\mu}{\sigma}\right)\right] \exp \left\{-\exp \left[\left(-\frac{x-\mu}{\sigma}\right)\right]\right\}
$$


One can link the type I to types II and III the following way: if the cdf of some random variable $X$ is of type II, and with the positive numbers as support, i.e., $G(x ; 0, \sigma, \xi)$, then the $\operatorname{cdf}$ of $\log X$ is of type I, namely $G(x ; \log \sigma, 1 / \xi, 0)$. Similarly, if the cumulative distribution function of $X$ is of type III, and with the negative numbers as support, i.e., $G(x ; 0, \sigma,-\xi)$, then the cdf of $\log (-X)$ is of type I, namely $G(x ;-\log \sigma, 1 / \xi, 0)$.

The unification of the original three families of extreme value distribution into a single family greatly simplifies statistical implementation. Through inference on $\xi$, the data themselves determine the most appropriate type of tail behaviour, and there is no necessity to make a priori about which individual extreme value family to adopt. Moreover, uncertainty in the inferred value of $\xi$ measures the lack of certainty as to which of the original three types is most appropriate for a given dataset.

Interpreting the limit in Fisher-Tippett-Gnedenko theorem as an approximation for large values of $n$, suggests the use of the GEV family for modeling the distribution of maxima of long sequences. The apparent difficulty that the normalizing constants will be unknown in practice, is easily resolved. Assuming (3-6),

$$
\mathbb{P}\left(\frac{M_{n}-b_{n}}{a_{n}}\right) \sim G(x)
$$

for large enough $n$. Equivalently,

$$
\mathbb{P}\left(M_{n} \leq x\right) \sim G\left(\frac{x-b_{n}}{a_{n}}\right)=: G^{*}(x),
$$

where $G^{*}$ is another member of the GEV family. In other words, if FisherTippett-Gnedenko theorem enables approximation of the distribution of $M_{n}^{*}$ by a member of the GEV family for large $n$, the distribution of $M_{n}$ itself, can be also approximated by a different member of the same family. Since the parameters of the distribution have to be estimated anyway, it is irrelevant in practice that the parameters of the distribution $G$ are different from those of $G^{*}$. 


\section{4}

\section{The Generalized Extreme Value Distribution for Minima}

Some applications require models for extremely small, rather than extremely large observations. Now we focus the statistical behaviour of

$$
\widetilde{M}_{n}:=\min \left\{X_{1}, \cdots, X_{n}\right\} .
$$

Assuming the $X_{i}$ to be independent and identically distributed, analogous arguments apply to $\widetilde{M}_{n}$ as were applied to $M_{n}$, leading to a limiting distribution of a suitably rescaled variable.

The results are also immediate from the corresponding results for $M_{n}$. Letting $Y_{i}=-X_{i}$ for $i=1, \ldots, n$, the change of sign means that small values of $X_{i}$ correspond to large values of $Y_{i}$. So if $\widetilde{M}_{n}=\min \left\{X_{1}, \ldots, X_{n}\right\}$ and $M_{n}=\max \left\{Y_{1}, \ldots, Y_{n}\right\}$, then $\widetilde{M}_{n}=-M_{n}$. Hence, for large $n$,

$$
\begin{aligned}
\mathbb{P}\left(\widetilde{M}_{n} \leq x\right) & =\mathbb{P}\left(M_{n} \geq-x\right) \\
& =1-\mathbb{P}\left(M_{n} \leq-x\right) \\
& =1-\exp \left\{-\left[1-\xi\left(\frac{x-\tilde{\mu}}{\sigma}\right)\right]^{-1 / \xi}\right\},
\end{aligned}
$$

on $\{x: 1-\xi(x-\tilde{\mu}) / \sigma>0\}$, where $\tilde{\mu}=-\mu$. Therefore, if there exist sequences of constants $\left\{a_{n} \in \mathbb{R}^{+}\right\}$and $\left\{b_{n} \in \mathbb{R}\right\}$ such that

$$
\lim _{n \rightarrow \infty} \mathbb{P}\left(\frac{\widetilde{M}_{n}-b_{n}}{a_{n}} \leq x\right)=: \widetilde{G}(x),
$$

for a non-degenerate distribution function $\tilde{G}$, then $\tilde{G}$ is a member of the family of distributions for minima:

$$
\tilde{G}(x)=1-\exp \left\{-\left[1-\xi\left(\frac{x-\tilde{\mu}}{\sigma}\right)\right]^{-1 / \xi}\right\},
$$

defined on the set $\{x: 1-\xi(x-\tilde{\mu}) / \sigma>0\}$, where the parameters satisfy $-\infty<\tilde{\mu}<\infty, \sigma>0$ and $-\infty<\xi<\infty$.

As an example, let $X_{i} \stackrel{d}{=} \operatorname{Exp}(\lambda)$ and $\widetilde{M}_{n}=\min \left\{X_{1}, \ldots, X_{n}\right\}$. Then it is easy to show that $\widetilde{M}_{n} \stackrel{d}{=} \operatorname{Exp}(n \lambda)$. In other words, if each of the $n$ independent variables $X_{i}$ is exponentially distributed with parameter $\lambda$, their minimal value is also exponentially distributed, but with parameter $n \lambda$. 


\section{5}

\section{Domains of Attraction}

In this section we state the necessary and sufficient criteria for the distribution function $P$ to belong to the domain of attraction of $G_{\xi}$, in which $\xi$ is the extreme value index of $G$, i.e.,

$$
\lim _{n \rightarrow \infty}\left(P\left(a_{n} x+b_{n}\right)\right)^{n}=G_{\xi}(x),
$$

for some given $\xi$ and all $x$.

Before stating the conditions, we make two points: firstly, the limit law of a sequence of random variables is uniquely determined up to changes of location and scale [37]. This means if there exist sequences $\left\{a_{n} \in \mathbb{R}^{+}\right\}$and $\left\{b_{n} \in \mathbb{R}\right\}$ such that

$$
\lim _{n \rightarrow \infty} \mathbb{P}\left(\frac{M_{n}-b_{n}}{a_{n}} \leq x\right)=G_{\xi}(x),
$$

then the relation

$$
\lim _{n \rightarrow \infty} \mathbb{P}\left(\frac{M_{n}-\beta_{n}}{\alpha_{n}} \leq x\right)=H_{\xi}(x),
$$

holds for sequences $\left\{\alpha_{n} \in \mathbb{R}^{+}\right\}$and $\left\{\beta_{n} \in \mathbb{R}\right\}$, iff

$$
\lim _{n \rightarrow \infty} \frac{a_{n}}{\alpha_{n}}=: \sigma \in \mathbb{R}^{+}, \quad \text { and } \quad \lim _{n \rightarrow \infty} \frac{b_{n}-\beta_{n}}{\alpha_{n}}=: \mu \in \mathbb{R} .
$$

In this case, $H(x)=G((x-\mu) / \sigma)$, and we say that $H$ and $G$ are of the same type. Thus, a distribution function $P$ can not be in the domain of attraction of $G_{\xi_{1}}$ and $G_{\xi_{2}}$ with $\xi_{1} \neq \xi_{2}$.

Furthermore, it may turn out that for a given distribution function $P$, there is no limit distribution $G$ such that $P \in \mathcal{D}(G)$. This simply means that the maximum $M_{n}$ does not have a nondegenerate limiting distribution under any linear rescaling. (a common example is the Poisson distribution [35]).

Now we are in a position to state the necessary and sufficient conditions for belonging to a domain of attraction. The Fréchet and Weibull cases are dealt with the help of slowly varying functions. Instead, the Gumbel case is more complex, for it would require the introduction of the von Mises functions (see, e.g., [38]); therefore, we only provide a sufficient condition [39]. These conditions, require the existence of one or two derivatives of $P$. 
The following theorem provides a characterization of the maximum domain of attraction: The distribution $P$ belongs to the domain of attraction for maxima of the family

1. Type I (Gumbel) if

$$
\lim _{x \rightarrow x_{+}}\left(\frac{1-P(x)}{P^{\prime}(x)}\right)^{\prime}=0 .
$$

2. Type II (Fréchet) iff $x_{+}=\infty$, and

$$
1-P(x)=x^{-\rho} \Psi(x), \quad \rho>0,
$$

for some slowly varying function $\Psi$ (see App. A).

3. Type III (Weibull) iff $x_{+}<\infty$, and

$$
1-P\left(x_{+}-x^{-1}\right)=x^{-\rho} \Psi(x), \quad \rho>0,
$$

for some slowly varying function $\Psi$. If we only consider the sufficient condition, we can reformulate the above theorem to unify the result: Let $P$ be a distribution function and $x_{+}$denotes the outer edge of its support and suppose $P^{\prime \prime}(x)$ exists and $P^{\prime}(x)$ is positive for all $x$ in some neighbourhood of $x_{+}$. If

or equivalently,

$$
\lim _{x \rightarrow x_{+}}\left(\frac{1-P(x)}{P^{\prime}(x)}\right)^{\prime}=\xi,
$$

$$
\lim _{x \rightarrow x_{+}} \frac{(1-P(x)) P^{\prime \prime}(x)}{\left(P^{\prime}(x)\right)^{2}}=-\xi-1,
$$

then $P \in \mathcal{D}\left(G_{\xi}\right)$.

Eventually, the sequences $\left\{a_{n}\right\}$ and $\left\{b_{n}\right\}$ can be calculated as follows:

1. Type I (Gumbel):

$$
b_{n}=P^{-1}(1-1 / n), \quad a_{n}=P^{-1}(1-1 / e n)-b_{n} .
$$

2. Type II (Fréchet):

$$
b_{n}=0, \quad a_{n}=P^{-1}(1-1 / n) .
$$

3. Type III (Weibull): 


$$
b_{n}=x_{+}, \quad a_{n}=P^{-1}(1-1 / n) .
$$

Here, $P^{-1}$ is the inverse cdf. The following a few examples show how choice of rescaling sequences does lead to a limit distribution within the GEV family, as implied by extermal types theorem.

\section{Example 1.}

If $X_{1}, X_{2}, \ldots$ is a sequence of independent standard exponential $\operatorname{Exp}(1)$ variables, then $P(x)=1-e^{-x}$ for $x>0$. In this case, letting $a_{n}=1$ and $b_{n}=\log n$,

$$
\begin{aligned}
\lim _{n \rightarrow \infty} \mathbb{P}\left(\frac{M_{n}-b_{n}}{a_{n}} \leq x\right) & =\lim _{n \rightarrow \infty}(P(x+\log n))^{n} \\
& =\lim _{n \rightarrow \infty}\left[1-e^{-(x+\log n)}\right]^{n} \\
& =\lim _{n \rightarrow \infty}\left[1-n^{-1} e^{-x}\right]^{n}=e^{-e^{-x}}
\end{aligned}
$$

for each fixed $x \in \mathbb{R}$. Hence, with the chosen $a_{n}$ and $b_{n}$, the limit distribution of $M_{n}$ as $n \rightarrow \infty$ is the Gumbel distribution, corresponding to $\xi=0$ in the GEV family.

\section{Example 2.}

If $X_{1}, X_{2}, \ldots$ is a sequence of independent standard Fréchet variables, then $P(x)=\exp (-1 / x)$ for $x>0$. Letting $a_{n}=n$ and $b_{n}=0$,

$$
\begin{aligned}
\lim _{n \rightarrow \infty} \mathbb{P}\left(\frac{M_{n}-b_{n}}{a_{n}} \leq x\right) & =\lim _{n \rightarrow \infty}(P(n x))^{n} \\
& =\lim _{n \rightarrow \infty}[\exp \{-1 /(n x)\}]^{n}=e^{-1 / x}
\end{aligned}
$$

for each fixed $x>0$. Hence, the limit in this case is also the standard Fréchet distribution with $\xi=1$ in the GEV family.

\section{Example 3.}

If $X_{1}, X_{2}, \ldots$ is a sequence of independent uniform variables, then 
$P(x)=x$ for $0 \leq x \leq 1$. For fixed $x$ suppose $n>-x$ and let $a_{n}=1 / n$ and $b_{n}=1$. Then,

$$
\begin{aligned}
\lim _{n \rightarrow \infty} \mathbb{P}\left(\frac{M_{n}-b_{n}}{a_{n}} \leq x\right) & =\lim _{n \rightarrow \infty}\left(P\left(n^{-1} x+1\right)\right)^{n} \\
& =\lim _{n \rightarrow \infty}\left(1+\frac{x}{n}\right)^{n}=e^{x}
\end{aligned}
$$

Hence, the limit distribution is of Weibull type, with $\xi=-1$ in the GEV family.

There is some freedom in the choice of $a_{n}$ and $b_{n}$ in such examples. However, different choices that lead to a non-degenerate limit, always yield a limit distribution in the GEV family with the same value of $\xi$, though possibly with other values of the location and scale parameters. 
4

\section{Introduction to Random Matrix Theory}

It is not knowledge, but the act of learning, not possession but the act of getting there, which grants the greatest enjoyment.

C. F. Gauss

In this chapter, we review the basic concepts of random matrix theory (RMT). After motivating RMT as powerful phenomenological models, we introduce the three classical ensembles along with the partition function associated with each one. Then, we discuss the important concept of universality in the context of RMT which provides the foundational basis for the applications of RMT in diverse areas.

\section{1}

\section{Motivation}

Except for textbook examples, problems in physics rarely have exact solution. Therefore, a physicist is often led to work with approximate "models" which may disregard certain details of the system in question. In fact, it is usually the case that, given the circumstances, one property of a physical system is more important than others and a full description of the system is not needed for answering the questions about that property. In certain situations, random matrix models are phenomenological models which provide partial solutions to wide variety of problems in physics and other branches of knowledge.

In the construction of a random matrix model for a given physical system, one starts with identifying a linear operator which carries information about the dynamics of the system. For example, this would usually be the Hamiltonian for a quantum mechanical system which one wants to study its spectrum. However, this might be difficult to be done analytically. 
Random matrix theory proceeds by identifying fundamental symmetries and constraints about the structure of this linear operator. For example, it may be complex Hermitian or real symmetric, or it might posses a certain block structure, or might have commutativity properties with other operators. Once important symmetries and constraints are identified, all other system specific information is "erased" from the model.

Discarding of information essentially corresponds to taking irrelevant details to be random. Thus the actual linear operator which contains full dynamical information is replaced by an ensemble of operators (matrices) which are random, except for the imposed constraints and symmetries on them. In most cases, an average over such an ensemble is much easier to evaluate than computing the spectrum for the original problem.

Dyson called random matrix theory as a

"new kind of statistical mechanics, in which we renounce the exact knowledge not of the state of a system, but of the nature of the system itself [3]".

In ordinary statistical mechanics one typically considers a collection of identical dynamical systems realized at different points in the phase space. The averages therefore are over those points (with respect to the Boltzmann weight). However, in random matrix theory the averaging is done over an ensemble of different dynamical systems (as represented by different linear operators) sharing the same symmetries and constraints. Ensemble averages are performed as a weighted sum over this set of dynamical systems. This kind of averaging wipes out any system specific information. Only those properties that are shared by almost all dynamical systems in the ensemble survive the averaging procedure. We refer to these properties as universal properties.

\section{2}

\section{Classical Ensembles of Random Matrices}

A random matrix model is defined by:

- An ensemble $E$ of $N \times N$ matrices $M$.

- A (not necessarily normalized) probability measure $\mathrm{d} \mu(M)$ for $M \in E$, invariant under the action of some symmetry group $G$. 
In order to be invariant (under $G$ ), the probability measure should depend only on the invariants of the matrix $M$, i.e., the traces of the matrix and its powers $\operatorname{Tr} M^{k}, k=1, \ldots, N$. To be more precise, according to a theorem due to Weyl [40], all the invariants of a $N \times N$ matrix $M$ (including any other $\operatorname{Tr} M^{k}, k>N$ and the determinant) can be expressed in terms of the traces of the first $N$ powers of $M$ $[41,42]$. The simplest case which is of practical use is of the form ${ }^{1}$

$$
\mathrm{d} \mu(M)=e^{-N \operatorname{Tr} V(M)} \mathrm{d} M
$$

i.e., a Boltzmann weight times an integration measure. The potential $V$ is often chosen to be a polynomial. Then, the quantity which contains almost all the information about the model is the partition function

$$
Z_{N}:=\int_{E} \mathrm{~d} \mu(M)=\int_{E} \mathrm{~d} M e^{-N \operatorname{Tr} V(M)}
$$

which is also invariant under $G$. Due to the symmetry under $G$, a matrix $M$ in the ensemble can be diagonalized as

$$
M=U \Lambda U^{-1}, \quad \Lambda \equiv \operatorname{diag}\left(\lambda_{1}, \ldots, \lambda_{N}\right) .
$$

This is called angular-radial decomposition or spectral decomposition, where $\Lambda$ is the radial part and $U$ is the angular part.

Local statistical properties of the spectrum such as level spacing are universal: they depend only on the symmetry of the ensemble to which $M$ belongs and do not depend on the probability measure. So they were originally studied using Gaussian measures, and the ensembles were called Gaussian ensmbles. Indeed, Dyson came up with a classification of generic ensembles in the framework of non-relativistic quantum mechanics:

- The Gaussian Orthogonal Ensemble (GOE) of real symmetric matrices.

- The Gaussian Unitary Ensemble (GUE) of complex Hermitian matrices.

- The Gaussian Symplectic Ensemble (GSE) of quaternionic Hermitian matrices.

Analyzing the consequences of the invariance under time-inversion $T$, Dyson showed [3-7] that the previous classes of random matrices can describe a system which

- is $T$-invariant and rotational invariant or with integer magnetic moment.

- is not $T$-invariant, e.g., with a magnetic field without other discrete symmetries.

\footnotetext{
${ }^{1}$ Assuming the statistical independence, excludes everything except the traces of the first two powers of $M$, which in turn, may occur only in exponential form [41].
} 
- is $T$-invariant and with half-integer magnetic moment.

Correspondingly, the matrix $U$ in (4-3) belongs to a compact Lie group which is called a circular ensemble:

- The Circular Orthogonal Ensemble (COE) of real symmetric matrices.

- The Circular Unitary Ensemble (CUE) of complex Hermitian matrices.

- The Circular Symplectic Ensemble (CSE) of complex symplectic matrices.

The Gaussian ensembles are denoted as $E_{N}^{\beta}$ and the Corresponding circular ensembles as $U_{N}^{\beta}$, with $\beta \in\{1,2,4\}$ is called Dyson index (see Tab. 4.1).

\begin{tabular}{|lc||lc|ll|}
\hline$\beta$ & Ensemble type & $E_{N}^{\beta}$ & Gaussian ensemble & $U_{N}^{\beta}$ & Circular ensemble \\
\hline \hline 1 & orthogonal & $S_{N}$ & GOE & $O_{N}$ & COE \\
\hline 2 & unitary & $H_{N}$ & GUE & $U_{N}$ & CUE \\
\hline 4 & symplectic & $Q_{N}$ & GSE & $S p_{2 N}$ & CSE \\
\hline
\end{tabular}

Table 4.1: Gaussian and circular ensembles.

Each one of these ensembles can be realized as a set of $N \times N$ matrices whose entries are real if $\beta=1$, complex if $\beta=2$, and quaternionic if $\beta=4$. The entries can be written as elements of a $\beta$-dimensional Clifford algebra over $\mathbb{R}$, with generators $\left\{\boldsymbol{e}_{\nu}\right\}_{\nu=0}^{\beta-1}$, in which $\boldsymbol{e}_{0}=\mathbf{1}$ is the identity and $\left\{\boldsymbol{e}_{i}\right\}_{i=1}^{3}$ satisfy

$$
\boldsymbol{e}_{i}^{2}=-\mathbf{1}, \quad \boldsymbol{e}_{i} \boldsymbol{e}_{j}=\epsilon_{i j k} \boldsymbol{e}_{k} .
$$

We recall that the conjugate of a quaternion $q \in$ of the form

is defined as

$$
q=\sum_{\nu=0}^{\beta-1} q^{(\alpha)} \boldsymbol{e}_{\alpha}, \quad q^{(\alpha)} \in \mathbb{R}
$$

$$
\bar{q}:=q^{(0)} \mathbf{1}-\sum_{i=1}^{\beta-1} q^{i} \boldsymbol{e}_{i} .
$$

Defining the conjugate of a matrix $M$ by $M^{\dagger}:=\bar{M}^{T}$, one can see the Gaussian ensembles satisfy the constraint $M^{\dagger}=M$, and the circular ensembles satisfy $M M^{\dagger}=\mathbb{1}$. Furthermore, in the case of quaternionic ensembles, one may represent the generators $\boldsymbol{e}_{i}$ by Pauli matrices; i.e., $\boldsymbol{e}_{i}=-i \sigma_{i}$ in which

$$
\sigma_{1}=\left(\begin{array}{cc}
0 & 1 \\
1 & 0
\end{array}\right), \quad \sigma_{2}=\left(\begin{array}{cc}
0 & -i \\
i & 0
\end{array}\right), \quad \sigma_{3}=\left(\begin{array}{cc}
1 & 0 \\
0 & -1
\end{array}\right)
$$

On each of the Gaussian ensembles $E_{N}^{\beta}$, there is an invariant Lebesgue measure, also called Haar measure which is the product of the Lebesgue measures 
on the real components of the matrix $M$. Denoting the real components of $M_{i j}$ as $M_{i j}^{(\alpha)}$, the Lebesgue measures are: ${ }^{2}$

$$
\mathrm{d} M= \begin{cases}\prod_{i} \mathrm{~d} M_{i i} \prod_{i<j} \mathrm{~d} M_{i j}, & \beta=1, \\ \prod_{i} \mathrm{~d} M_{i i} \prod_{i<j} \mathrm{~d}\left(\Re M_{i j}\right) \mathrm{d}\left(\Im M_{i j}\right), & \beta=2, \\ \prod_{i} \mathrm{~d} M_{i i}^{(0)} \prod_{i<j} \prod_{\alpha=0}^{3} \mathrm{~d} M_{i j}^{(\alpha)}, & \beta=4,\end{cases}
$$

which can be collected in a single formula

$$
\mathrm{d} M=\prod_{i} \mathrm{~d} M_{i i} \prod_{i<j} \prod_{\alpha=0}^{\beta-1} \mathrm{~d} M_{i j}^{(\alpha)} .
$$

The diagonalization (4-3) can be viewed as a change of variable $M \rightarrow(\Lambda, U)$; therefore, the Lebesgue measure $\mathrm{d} M$ can be written in terms of the measures on $\Lambda$ and $U$ as

$$
\mathrm{d} M=|\Delta(\Lambda)|^{\beta} \mathrm{d} \Lambda \mathrm{d} U_{\text {Haar }}
$$

where $\mathrm{d} \Lambda=\prod_{i=1}^{N} \mathrm{~d} \lambda_{i}$ is the Lebesgue measure on $\mathbb{R}^{N}$ and the Jacobian is written in terms of the Vandermonde determinant:

$$
\begin{aligned}
\Delta(\Lambda) \equiv \operatorname{det}\left(\lambda_{i}^{j-1}\right)_{1 \leq i, j \leq N} & =\left|\begin{array}{ccccc}
1 & \lambda_{1} & \lambda_{1}^{2} & \cdots & \lambda_{1}^{N-1} \\
1 & \lambda_{2} & \lambda_{2}^{2} & \cdots & \lambda_{2}^{N-1} \\
\vdots & \vdots & \vdots & \ddots & \vdots \\
1 & \lambda_{N} & \lambda_{N}^{2} & \cdots & \lambda_{N}^{N-1}
\end{array}\right| \\
& =\prod_{1 \leq i<j \leq N}\left(\lambda_{j}-\lambda_{i}\right),
\end{aligned}
$$

and $\mathrm{d} U_{\text {Haar }}$ is the Haar measure on the circular ensemble $U_{N}$ which is characterized as being invariant under the left and right action of the corresponding Lie group. ${ }^{3}$ The partition function (4-2) thus becomes

${ }^{2}$ In fact, one defines an invariant Hilbert-Schmidt inner product for two operators $A$ and $B$ on a Hilbert space $\mathcal{H}$ as $\langle A, B\rangle_{H S}:=\operatorname{Tr}\left(A^{\dagger} B\right)$ which induces the invariant measure (4-9), possessing the property $\mathrm{d} M=\mathrm{d}\left(U^{-1} M U\right)$.

${ }^{3}$ The left (right) Haar measure $\mathrm{d} U_{H a a r}^{L(R)}$ on a compact Lie group $U_{N}$, is an infinitesimal volume element of $U_{N}$ under which the integral over the whole group of some continuous function $f$ is invariant under left (right) group multiplication, i.e., for $U, U^{\prime} \in U_{N}$,

$$
\begin{gathered}
\int_{U_{N}} \mathrm{~d} U_{\text {Haar }}^{L} f\left(U^{\prime} U\right)=\int_{U_{N}} \mathrm{~d} U_{\text {Haar }}^{L} f(U), \\
\int_{U_{N}} \mathrm{~d} U_{\text {Haar }}^{R} f\left(U U^{\prime}\right)=\int_{U_{N}} \mathrm{~d} U_{\text {Haar }}^{R} f(U) .
\end{gathered}
$$

Therefore, the Haar measure assigns an "invariant volume" to subsets of compact Lie groups. It is clear that for consistency with the definition of integration, the Haar measure must be used when integrating over the angular variables. 


$$
Z_{N}=\operatorname{Vol}\left(\frac{E_{N}^{\beta}}{\mathbb{R}^{N}}\right) \int_{\mathbb{R}^{N}} \mathrm{~d} \Lambda|\Delta(\Lambda)|^{\beta} e^{-N \operatorname{Tr} V(\Lambda)},
$$

in which $\operatorname{Vol}\left(\frac{E_{N}^{\beta}}{R^{N}}\right)$ is the volume of the flag manifold given by

$$
\operatorname{Vol}\left(\frac{E_{N}^{\beta}}{\mathbb{R}^{N}}\right)= \begin{cases}\frac{2^{N} \pi^{N(N+1) / 4}}{N ! \prod_{K=1}^{N} \Gamma(k / 2)}, & \beta=1, \\ \frac{\pi^{N(N-1) / 2}}{N ! \prod_{K=0}^{N-1} \Gamma(k+1)}, & \beta=2, \\ \frac{\pi^{N(N-1)}}{N ! \prod_{K=0}^{N-1} \Gamma(2 k+2)}, & \beta=4 .\end{cases}
$$

\section{3}

\section{Universality of Random Matrix Theories}

In spite of that random matrix models are extremely simple, they can be used to reproduce certain features of the real spectra of physical systems. The reason behind this applicability is universality.

The concept of universality was originally introduced in physics for the description of critical phenomena in statistical physics. The observation that critical exponents are quite insensitive to many characteristics of a physical system, gave rise to several experimental and theoretical efforts. Within that framework, the term universality means that there are observables which are mainly independent of any microscopic details of the system, but which show a very marked dependence both on symmetry and on the number of space dimensions. This simple idea turned out to be so fruitful that it spread out over several branches of theoretical physics, involving in particular those related to the study of complex systems. Random matrix theory is a remarkable example.

Within the context of random matrix theory, universality has a rather informal meaning: there is no generally accepted formal definition of universality in random matrix theory; However, it is generally agreed that, to call a property universal it should not depend on the details of the probability measure imposed on the ensemble. We recall that (see Sec. 4.2) the definition of a random matrix ensemble not only requires the choice of a subset among matrices (i.e., universality class), but also to assign a probability with respect to which all averages should be performed. One typically works with Gaussian distributions as they are easier to handle; however, for the outcomes of the model to have any significance, the arbitrary choice of a weight function should not have any role. More precisely, rewriting the partition function

$$
Z_{N}=\int_{E_{N}^{\beta}} \mathrm{d} M e^{-N \operatorname{Tr} V(M)}
$$


for a given ensemble $E_{N}^{\beta}$, universality then means independence from the choice of the potential $V$. Once a property is known to be universal, it can be studied using a quadratic potential, which leads to a Gaussian matrix integral. In fact, Universality arises in limit, where the matrix size $N$ is large. Then, assuming that the potential $V$ has a finite large $N$ limit, the partition function $Z_{N}$ can have a non-trivial large $N$ limit too:

$$
\lim _{N \rightarrow \infty} Z_{N}=: Z
$$

In fact, in order for this limit to exist, the action term must be of the same order as the integration measure. If its order is larger, that means the measure is irrelevant and the matrix is not random, rather it is trapped at the bottom of the potential. On the other hand, if its order is smaller, the potential is irrelevant, and the matrix is free at limit $N \rightarrow \infty$ (see Sec. 5.1).

This form of universality is reminiscent of the central limit theorem. Like the central limit theorem, universality studies in the context of random matrix theory, establish certain spectral distributions to be invariant under changes of the defining probability distribution for the ensemble in question. However, unlike in the case of the central limit theorem, general proofs are harder to provide by and usually each variation of a model, requires separate study.

\subsection{1}

\section{Level Spacing Distribution}

Statistical study of energy levels is a rather unusual approach. A Hamiltonian, though it may be unknown or complicated, deterministically specifies the energy levels of a system and there is no uncertainty involved. However, if one is not concerned with the exact positions of energy levels, one might hope to understand the relations between levels within a certain level of precision, such as correlations or the average spectral density. Thus Wigner considered the spectrum of a random matrix which shares the symmetries of the given Hamiltonian.

The interesting observable which Wigner studied $[43,44]$ is the statistical distribution of the distance $s$ between adjacent energy levels which appear in experimental data as peaks of the diffusion rate of neutrons as a function of the energy in the scattering of slow neutrons across nuclei (see Fig. 4.1).

More precisely, let $\lambda_{1}<\cdots<\lambda_{n}<\lambda_{n+1}<\cdots$ be the ordered sequence of energy levels. One defines the normalized spacings $s:=\left(\lambda_{n+1}-\lambda_{n}\right) / \mathbb{E}[s]$, where $\mathbb{E}[s]=\mathbb{E}\left[\lambda_{n+1}-\lambda_{n}\right]$ is the mean spacing. If the energy levels were uncorrelated random numbers, that is, the levels were distributed at random, the variable $s$ 


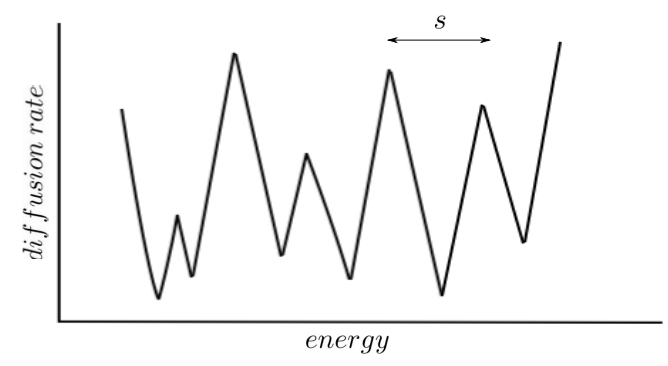

Figure 4.1: Diffusion rate of neutrons as a function of the energy. The distance between neighbouring energy levels is denoted by $s$.

would be governed by the Poisson distribution [41], with probability density 4

$$
p(s)=e^{-s} .
$$

But the experimentally observed probability density looks quite different, and is very well approximated (within 1\%) by the Wigner surmise [45]

$$
p_{\beta}(s)=a_{\beta} s^{\beta} e^{-b_{\beta} s^{2}},
$$

where the parameter $\beta \in\{1,2,4\}$ is determined by the symmetries of the nucleus under time reversal and spin rotation, and the values of $a_{\beta}$ and $b_{\beta}$ are fixed by normalizing $p_{\beta}$ and the average value of $s$, i.e.,

$$
\int_{0}^{\infty} \mathrm{d} s p_{\beta}(s)=1=\int_{0}^{\infty} \mathrm{d} s p_{\beta}(s) s
$$

In fact, Wigner surmise for the three classical ensembles is (see Fig. 4.1)

$$
p_{\beta}(s)= \begin{cases}\frac{\pi}{2} s e^{-\frac{\pi}{4} s^{2}}, & \beta=1, \\ \frac{32}{\pi^{2}} s^{2} e^{-\frac{4}{\pi} s^{2}}, & \beta=2, \\ \frac{2^{18}}{3^{6} \pi^{3}} s^{4} e^{-\frac{64}{9 \pi} s^{2}}, & \beta=4 .\end{cases}
$$

The Wigner surmise is also a very good approximation of the large size limit of the probability density for the distance between consecutive eigenvalues of random matrices. Actually, this density is universal, in the sense that it does not depend on the precise probability law of the random matrices, but only on the choice of the matrix ensemble.

Nearest neighbour spacing in an energy spectrum and distance between consecutive eigenvalues in the spectrum of random matrices are two examples of

${ }^{4}$ From characteristics of the Poisson process, spacings between consecutive energy levels act as sequences of independent random variables. By the memoryless property of the Poisson process (see Ch. 2), it also means that the number of energy levels with one particular spacing is independent of the number of those with some other spacing. 


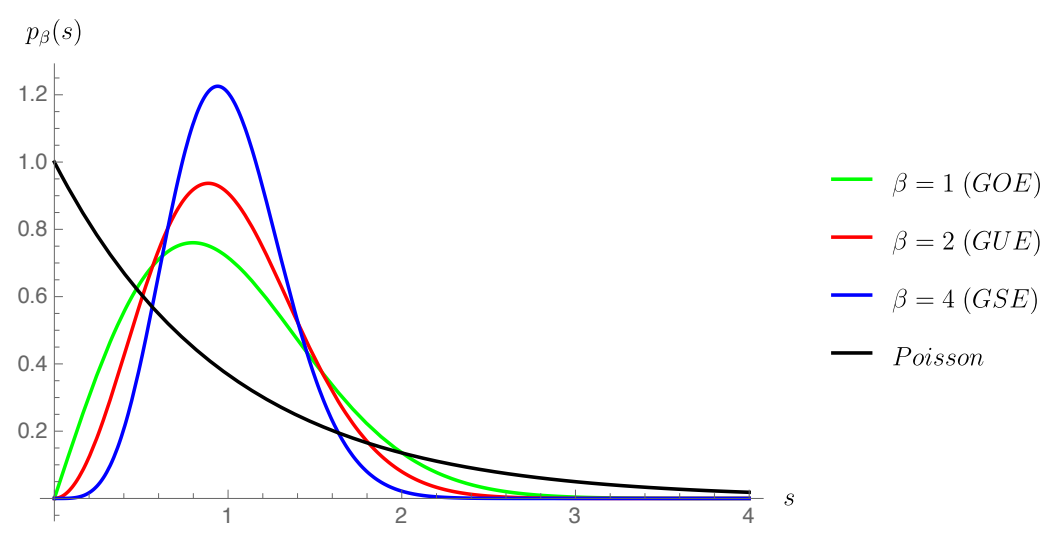

Figure 4.2: Wigner surmise for level spacing probability distribution.

local properties of the corresponding spectrum. By local properties we mean those properties that are relevant at the scale of the mean eigenvalue spacing.

\subsection{2}

\section{Eigenvalue Distribution}

On the other hand, the density of the eigenvalues (as opposed to their distances) is not universal. In the case of Gaussian random matrices in the limit of infinite size, it is given by the Wigner semi-circle law [46]

$$
\rho(\lambda)=\frac{1}{2 \pi} \sqrt{4-\lambda^{2}} \mathbb{1}_{\{|\lambda| \leq 2\}} .
$$

However, for many physical systems, the average spectral density is a monotonically increasing function of the energy. Therefore it is clear that such an approach cannot say much about the overall shape of the spectral density; in other words, the details of the system ignored by the random matrix model are important for the determination of the average spectral density. 


\section{5}

\section{Gaussian Unitary Ensemble}

The beauty of mathematics only shows itself to more patient followers.

M. Mirzakhani

All information concerning the distribution of eigenvalues in any of the classical ensembles (GOE, GUE, and GSE) is encoded in the jpdf. Getting information out of the jpdf requires integration, and many of the integrals that arise can not be evaluated in any nice closed form. Nevertheless, it is possible to show that as the size $N$ of the ensemble becomes large, certain interesting functions of the eigenvalues; for instance, the maximum, have (after suitable re-centering and re-scaling) limit distributions. The unitary ensembles are easier to handle than the orthogonal and symplectic ones, so we will limit our attention to GUE in this chapter:

\section{1}

\section{Spectral Representation of the Partition Function of GUE}

According to the previous chapter, the partition function of GUE is given by

$$
Z=\int_{H_{N}} \mathrm{~d} M e^{-N \operatorname{Tr} V(M)}
$$

in which $V(M)=\frac{1}{2} M^{2}$ and the integration measure is the following Lebesgue measure

$$
\mathrm{d} M=\prod_{i=1}^{N} \mathrm{~d} M_{i i} \prod_{1 \leq i<j \leq N} \mathrm{~d}\left(\Re M_{i j}\right) \mathrm{d}\left(\Im M_{i j}\right) .
$$

which is invariant under the conjugation

$$
M \rightarrow U^{-1} M U=: \Lambda, \quad U \in U(N),
$$

in which $\Lambda:=\operatorname{diag}\left(\lambda_{1}, \ldots, \lambda_{N}\right) \in \mathbb{R}^{N}$ includes the (real) eigenvalues of $M$, and $U$ is a unitary matrix. Along with the invariance of the Lebesgue measure, the cyclic property of the trace, i.e.,

$$
\operatorname{Tr} M=\operatorname{Tr}\left(U M U^{-1}\right)
$$

implies the invariance of the partition function $Z$ in (5-1) under the symmetry group $U(N)$. 
Moreover, the total number of degrees of freedom is $N^{2}$. The Lebesgue measure $\mathrm{d} M$ is the product of Lebesgue measures for the $N^{2}$ real components, i.e., of order $O\left(e^{N^{2}}\right)$. The trace is a sum of $N$ terms, and $e^{-N \operatorname{Tr} V(M)}=O\left(e^{-N^{2}}\right)$, thanks to the pre-factor $N$. Therefore, the partition function $Z$ has a non-trivial large $N$ limit.

Implementing the symmetry (5-3), one can integrate over the unitary part $U$ of $M$ and the measure reduces to the measure over the diagonal part (the eigenvalues). Then, one has to compute the Jacobian $J=\frac{\partial M}{\partial(U, \Lambda)}$ of the transformation $M \rightarrow$ $(U, \Lambda)$ :

$$
\mathrm{d} M=J \mathrm{~d} \Lambda \mathrm{d} U_{\text {Haar }} .
$$

Since $\mathrm{d} M$ is invariant under a unitary transformation, the Jacobian $J$ depends only on $\Lambda$, and it is sufficient to calculate it in the vicinity of $U=\mathbb{1}$. Differentiating (5-3) we have

$$
\mathrm{d} M=(\mathrm{d} U) \Lambda U^{-1}+U(\mathrm{~d} \Lambda) U^{-1}+U \Lambda\left(\mathrm{d} U^{-1}\right)
$$

with the additional constraint

$$
\mathrm{d}\left(U U^{-1}\right)=(\mathrm{d} U) U^{-1}+U\left(\mathrm{~d} U^{-1}\right)=0 .
$$

Hence, for $i=j$ : $\mathrm{d} M_{i i}=\mathrm{d} \lambda_{i}$, and for $i<j: \mathrm{d}\left(\Re M_{i j}\right)=\left(\lambda_{i}-\lambda_{j}\right) \mathrm{d}\left(\Re U_{i j}\right)$, and $\mathrm{d}\left(\Im M_{i j}\right)=\left(\lambda_{i}-\lambda_{j}\right) \mathrm{d}\left(\Im U_{i j}\right)$. Therefore, the Jacobian is simply $[7,41,47]$

$$
J=|\Delta(\Lambda)|^{2}
$$

in which $\Delta(\Lambda) \equiv \prod_{1 \leq i<j \leq N}\left(\lambda_{j}-\lambda_{i}\right)$ is the Vandermonde determinant. After integrating over the unitary part, one obtains the eigenvalue representation of the partition function ${ }^{1}$

$$
Z_{N}=\Omega_{N} \int_{\mathbb{R}} \prod_{i=1}^{N} \mathrm{~d} \lambda_{i}\left|\Delta\left(\lambda_{1}, \ldots, \lambda_{N}\right)\right|^{2} e^{-N \sum_{i=1}^{N} V\left(\lambda_{i}\right)} .
$$

in which

${ }^{1}$ We recall that in the context of group theory, Haar measure is a way to assign "invariant volume" to subsets of compact groups and subsequently define an integral for functions on those groups. Here,

$$
\Omega_{N}=\frac{\operatorname{Vol}(U(N))}{\left|S_{N}\right|(\operatorname{Vol}(U(1)))^{N}}=\frac{1}{N !} \operatorname{Vol}\left(\operatorname{Flag}^{(N)}(\mathbb{C})\right),
$$

where $\left|S_{N}\right|=N$ ! is the order of the symmetry group $S_{N}$, and $\operatorname{Vol}(U(N))=\prod_{k=1}^{N} \frac{2 \pi^{k}}{(k-1) !}$ denotes the volume of the unitary group $U(N)$ with respect to the Haar measure on this group [48-51]. The eigenvalue permutation prefactor $N$ ! reflects the ambiguity of the representation $M=U \Lambda U^{-1}$. 


$$
\Omega_{N} \equiv \frac{1}{(2 \pi)^{N} N !} \int_{U(N)} \mathrm{d} U_{H a a r}=\frac{\pi^{N(N-1) / 2}}{\prod_{k=1}^{N} k !}
$$

is the volume of the flag manifold $[51,52]$.

More generally, we are interested in global or in local statistics of the eigenvalues. Global quantities involve a large number, e.g., $O(N)$, of eigenvalues like the expectation values of functions of the $\lambda_{i}$ 's (observables of RMT): ${ }^{2}$

$$
\mathbb{E}[f(M)]:=\frac{1}{Z_{N}} \int \mathrm{d} M e^{-N \operatorname{Tr} V(M)} f(M),
$$

with $f$ invariant under $M \rightarrow U M U^{-1}$. Such $f$ is expressible in terms of eigenvalues and we have ${ }^{3}$

$$
\mathbb{E}[f(\Lambda)]=\frac{1}{Z_{N}} \int_{\mathbb{R}} \mathrm{d} \Lambda|\Delta(\Lambda)|^{2} e^{-N \operatorname{Tr} V(\Lambda)} f(\Lambda) .
$$

Indeed the Gaussian unitary ensemble is exactly solvable and the partition function $Z$ and the expectation values $\mathbb{E}[f]$ can be computed explicitly [53]. For example, using Lebesgue measure (5-2), the partition function $Z$ reads

$$
Z_{N}=2^{N / 2}\left(\frac{\pi}{N}\right)^{N^{2} / 2}
$$

\section{2}

\section{Methods of Analysis the Large $N$ Limit}

Based on the eigenvalue representation (5-9) of the partition function, we discuss two different methods for analyzing the large $N$ limit of GUE.

\subsection{1}

\section{Saddle Point Method for GUE}

The starting point of this method is to notice that one can rewrite the eigenvalue representation of the partition function (5-9), omitting the prefactor $\Omega_{N}$, as

$$
Z_{N}=\int_{\mathbb{R}} \prod_{i=1}^{N} \mathrm{~d} \lambda_{i} e^{-N^{2} S_{e f f}\left(\lambda_{1}, \ldots, \lambda_{N}\right)}
$$

where the effective action is given by

$$
S_{e f f}\left(\lambda_{1}, \ldots, \lambda_{N}\right):=\frac{1}{N} \sum_{i=1}^{N} \frac{\lambda_{i}^{2}}{2}-\frac{2}{N^{2}} \sum_{1 \leq i<j \leq N} \ln \left|\lambda_{i}-\lambda_{j}\right| .
$$

Equation (5-15) (or equivalently (5-9)) exhibits a fundamental feature: due to the presence of the Jacobian, given in terms of Vandermonde determinant, eigenvalues

${ }^{2}$ Here $\mathbb{E}[\cdot]$ means average over systems with different Hamiltonians (potentials) but with common global symmetries, in contrast to the traditional ensemble average of identical physical systems (with fixed Hamiltonian) in statistical mechanics.

${ }^{3}$ In the language of group theory, $f$ is called a calss function. 
can not coincide. This is referred to as repulsion of eigenvalues of random matrices. Alternatively (5-15) may be regarded as describing a classical statistical mechanical model of $N$ charges on the real line, subjected to a one-body potential $N \lambda^{2} / 2$ and to a repulsive interaction which has the form of a $2 D$ Coulomb repulsion. Assuming the potential to be $N$-independent and all eigenvalues to be of order one, the sum over $N$ eigenvalues is roughly of order one. Therefore $N^{2} S_{\text {eff }}=O\left(N^{2}\right)$, and in the limit $N \rightarrow \infty$, the integral (5-14) will be dominated by a saddle point configuration that minimizes the effective action, i.e., we have to look for the solution $\left(\tilde{\lambda}_{1}, \ldots, \tilde{\lambda}_{N}\right)$ of the following set of equations:

$$
\left.\frac{\delta}{\delta \lambda_{i}} S_{e f f}\left(\lambda_{1}, \ldots, \lambda_{N}\right)\right|_{\lambda_{i}=\tilde{\lambda}_{i}}=0, \quad i=1, \ldots, N,
$$

which leads to the saddle point equation:

$$
\tilde{\lambda}_{i}=\frac{2}{N} \sum_{j=1}^{N} \frac{1}{\tilde{\lambda}_{i}-\tilde{\lambda}_{j}}, \quad i=1, \ldots, N .
$$

In this saddle point configuration $\lambda_{i}=\tilde{\lambda}_{i}$, the eigenvalue density formally defined for finite $N$ as

$$
\rho_{N}(\lambda):=\mathbb{E}\left[\frac{1}{N} \operatorname{Tr} \delta(\lambda \mathbb{1}-M)\right]=\mathbb{E}\left[\frac{1}{N} \sum_{i=1}^{N} \delta\left(\lambda-\lambda_{i}\right)\right],
$$

becomes

$$
\rho_{N}(\lambda)=\frac{1}{N} \sum_{i=1}^{N} \delta\left(\lambda-\tilde{\lambda}_{i}\right) .
$$

In this configuration, the large $N$ limit of the partition function reads

$$
Z_{N} \approx e^{-N^{2} S_{e f f}\left(\tilde{\lambda}_{1}, \ldots, \tilde{\lambda}_{N}\right)}
$$

More generally, any invariant expectation value of the form (5-11) is computable by the saddle point method:

$$
\langle f(M)\rangle \approx f\left(\tilde{\lambda}_{1}, \ldots, \tilde{\lambda}_{N}\right) .
$$

In the large $N$ limit, it is reasonable to expect that the discrete eigenvalue distribution (which is an observable) becomes a continuous function

$$
\rho(\lambda):=\lim _{N \rightarrow \infty} \rho_{N}(\lambda)
$$

with compact support. Therefore, we assume that $\rho(\lambda)$ vanishes outside a real interval $S:=\operatorname{supp}(\rho)=\left[c_{-}, c_{+}\right]$. This is the so-called one-cut solution. Furthermore, as there are exactly $N$ eigenvalues on the real axis, the density of eigenvalues is subject to the normalization condition

$$
\int_{\mathcal{S}} \mathrm{d} \lambda \rho(\lambda)=1
$$


We then can write the continuum limit of the equations of our matrix model in terms of continuous quantities, by using the standard rule

$$
\frac{1}{N} \mathbb{E}\left[\sum_{i=1}^{N} f\left(\lambda_{i}\right)\right]=\frac{1}{N} \sum_{i=1}^{N} f\left(\tilde{\lambda}_{i}\right) \rightarrow \int_{\mathcal{S}} \mathrm{d} \lambda \rho(\lambda) f(\lambda) .
$$

For example, the effective action in the continuum limit is given by

$$
S_{e f f}[\rho]=\frac{1}{2} \int_{\mathcal{S}} \mathrm{d} \lambda \rho(\lambda) \lambda^{2}-f_{\mathcal{S} \times \mathcal{S}} \mathrm{d} \lambda \mathrm{d} \lambda^{\prime} \rho(\lambda) \rho\left(\lambda^{\prime}\right) \ln \left|\lambda-\lambda^{\prime}\right|,
$$

Therefore, in this large $N$ limit, the eigenvalue density is such that the effective action (5-25) attains its minimum, while the constraint (2-12) is imposed. We then have to solve the following variational problem:

$$
\begin{aligned}
\frac{\delta}{\delta \rho(\lambda)}\left[\frac{1}{2} \int_{\mathcal{S}} \mathrm{d} \lambda^{\prime} \rho\left(\lambda^{\prime}\right) \lambda^{\prime 2}-f_{\mathcal{S} \times \mathcal{S}} \mathrm{d} \lambda^{\prime} \mathrm{d} \lambda^{\prime \prime} \rho\left(\lambda^{\prime}\right) \rho\left(\lambda^{\prime \prime}\right) \ln \left|\lambda^{\prime}-\lambda^{\prime \prime}\right|\right. \\
\left.+c\left(\int_{\mathcal{S}} \mathrm{d} \lambda^{\prime} \rho\left(\lambda^{\prime}\right)-1\right)\right]=0
\end{aligned}
$$

in which $c$ is a constant. Applying the variational derivative in (5-26), leads to

$$
\frac{1}{2} \lambda^{2}=2 \int_{\mathcal{S}} \mathrm{d} \lambda^{\prime} \rho\left(\lambda^{\prime}\right) \ln \left|\lambda-\lambda^{\prime}\right|-c
$$

Next, we take derivative of both sides of the above equation with respect to $\lambda$, to eliminate $c$. Thereby, we have

$$
\lambda=2 \int_{-\infty}^{\infty} \mathrm{d} \lambda^{\prime} \frac{\rho\left(\lambda^{\prime}\right)}{\lambda-\lambda^{\prime}} .
$$

This is the continuum form of the saddle point equation (5-17), which is a singular integral equation that allows one, in principle, to calculate $\rho(\lambda)[54,55]$. The result is $[41]$

$$
\rho(\lambda)= \begin{cases}\frac{1}{2 \pi} \sqrt{4-\lambda^{2}}, & |\lambda|<2 \\ 0, & |\lambda|>2\end{cases}
$$

which is the celebrated Wigner's semi-circle law for the Gaussian model (see Fig. 5.1). The $2 n^{\text {th }}$ moment of this distribution is given by

$$
\omega_{2 n}=\int_{-2}^{2} \mathrm{~d} \lambda \frac{1}{2 \pi} \sqrt{4-\lambda^{2}} \lambda^{2 n}=C_{n}
$$

where $C_{n} \equiv \frac{1}{n+1}\left(\begin{array}{c}2 n \\ n\end{array}\right)$ is Catalan number. In fact, the most direct combinatoricsbased proof of the semi-circle law was given by Wigner in his original paper [43]. 


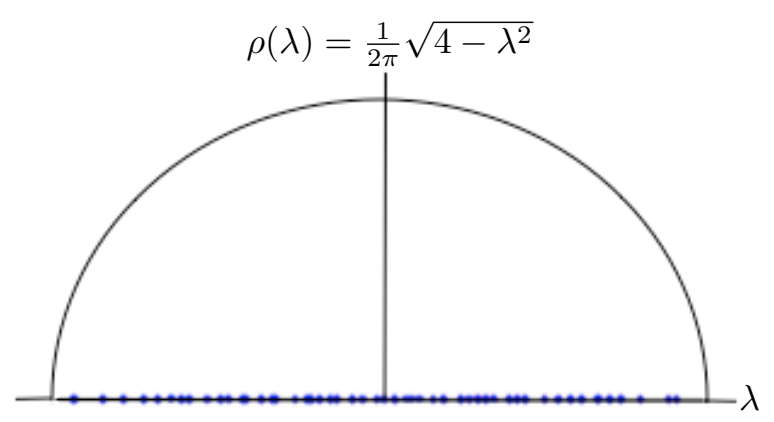

Figure 5.1: Wigner's semi-circle law for GUE.

\section{2 .2}

\section{Resolvent Formalism for Unitary Ensembles with Arbitrary Potential}

In the case of an arbitrary potential $V(\lambda)$, the saddle point equation (5-17), reads

$$
V^{\prime}\left(\tilde{\lambda}_{i}\right)=\frac{2}{N} \sum_{j=1}^{N} \frac{1}{\tilde{\lambda}_{i}-\tilde{\lambda}_{j}}, \quad i=1, \ldots, N .
$$

This means that the eigenvalues are subject to an effective potential

$$
V_{e f f}\left(\lambda_{i}\right)=V\left(\lambda_{i}\right)-\frac{2}{N} \sum_{1 \leq i<j \leq N} \ln \left|\lambda_{i}-\lambda_{j}\right| .
$$

At equilibrium, the eigenvalues tend to locate at the bottom of the potential. This means that in this case, the interval $S$ collapses to a point. The Coulomb repulsion, however, forces the eigenvalues to be apart from each other and spread out over an interval $\mathcal{S}$. The eigenvalues are then kept away from each other by a distance, typically of order $O(1 / N)$ (see Fig. 5.2).

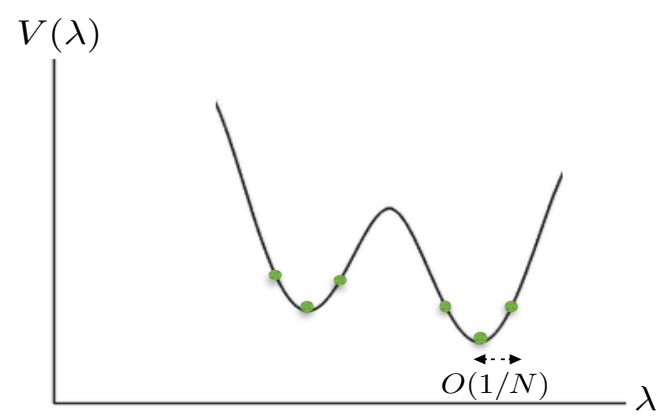

Figure 5.2: Repulsion of the Coulomb gas of eigenvalues.

The saddle point equation (5-31) in the continuum limit reads

$$
V^{\prime}(\lambda)=2 f_{\mathcal{S}} \mathrm{d} \lambda^{\prime} \frac{\rho\left(\lambda^{\prime}\right)}{\lambda-\lambda^{\prime}}, \quad \lambda \in \mathcal{S}
$$

This time, however, instead of applying the tedious methods of the theory of integral equations, we resort to the elegant and powerful methods of complex analysis which 
besides providing the solution, contain deep insight into its nature. The way to solve (5-33), is to introduce an auxiliary function $\omega(z)$, called resolvent, defined $a^{4}$

$$
\omega(z):=\frac{1}{N} \mathbb{E}\left[\operatorname{Tr} \frac{1}{z \mathbb{1}-M}\right]=\frac{1}{N} \mathbb{E}\left[\sum_{k=1}^{N} \frac{1}{z-\lambda_{k}}\right]=\frac{1}{N} \sum_{k=1}^{N} \frac{1}{z-\tilde{\lambda}_{k}} .
$$

The resolvent is thus the generating function for the moments of the measure defined by $\mathrm{d} \mu(\lambda):=\rho(\lambda) \mathrm{d} \lambda$, i.e.,

$$
\omega(z)=\sum_{k=0}^{\infty} \frac{1}{N} \mathbb{E}\left[\operatorname{Tr} M^{k}\right] z^{-k-1}
$$

where the expansion is valid for sufficiently large $|z|\left(|z|>\max \left\{\left|\lambda_{1}\right|, \ldots,\left|\lambda_{N}\right|\right\}\right)$. According to (5-35), the resolvent is $Z$-transform of the coefficient of the expansion, $\frac{1}{N} \mathbb{E}\left[\operatorname{Tr} M^{k}\right]$, the so called disc function, and therefore, the disc function is the inverse $Z$-transform of the resolvent, given by

$$
\frac{1}{N} \mathbb{E}\left[\operatorname{Tr} M^{k}\right]=: \omega_{k}=\frac{1}{2 \pi i} \oint_{C} \mathrm{~d} z z^{k} \omega(z),
$$

in which $C$ is a counterclockwise contour encircling the origin and all the poles of $\omega(z)$. In the continuum limit, when $N \rightarrow \infty$, the resolvent reads (see (5-34))

$$
\omega(z)=\int_{\mathcal{S}} \mathrm{d} \lambda \frac{\rho(\lambda)}{z-\lambda}, \quad z \notin \mathcal{S} .
$$

The resolvent is thus the Stieltjes transform ${ }^{5}$ of the measure $\mathrm{d} \mu(\lambda)=\rho(\lambda) \mathrm{d} \lambda$, and has two important properties:

- First, according to the equation (5-37), the integral has a singularity at $\lambda=z \in \mathcal{S}$. Therefore, analytic continuation of $\omega(z)$ into the complex plane is well-defined on $\mathbb{C} \backslash \mathcal{S}$. Since all the poles of $\omega(z)$ lie on the compact support $\mathcal{S} \subset \mathbb{R}$, forming a branch-cut on the real line, we can deform the contour $C$ in (5-36) to an infinitesimal rectangular contour encircling $\mathcal{S}$. Hence, we have

$$
\omega_{k}=\int_{\mathcal{S}} \mathrm{d} \lambda \lambda^{k} \rho(\lambda)
$$

- Second, based on the equation (5-35) and normalization of the eigenvalue distribution, it has the asymptotic behavior

${ }^{4}$ The resolvent formalism is a technique for applying concepts from complex analysis to the study of the spectrum of operators. The resolvent captures the spectral properties of an operator in the analytic structure of the resolvent.

${ }^{5}$ The Stieltjes transformation $S_{\rho}(z)$ of a continuous measure of density $\rho$ on a real interval $I$ is a function of the complex variable $z$ defined outside $I$ by the formula

$$
S_{\rho}(z):=\int_{I} \mathrm{~d} t \frac{\rho(t)}{z-t}, \quad t \in I, z \in \mathbb{C} \backslash I .
$$




$$
\omega(z) \underset{|z| \rightarrow \infty}{\sim} \frac{1}{z}
$$

One can now recover the density function $\rho$ by computing the discontinuity of $\omega(z)$ as one crosses the interval $\mathcal{S}$ (see Fig. 5.3). To this end, we consider the values $\omega^{ \pm}:=\omega(\lambda \pm i \epsilon)$, for $\lambda \in \mathcal{S}$ and $\epsilon \in \mathbb{R}^{+}$, and use a version of the SokhotskiWeierstrauss theorem [56] for integrals over real line which states

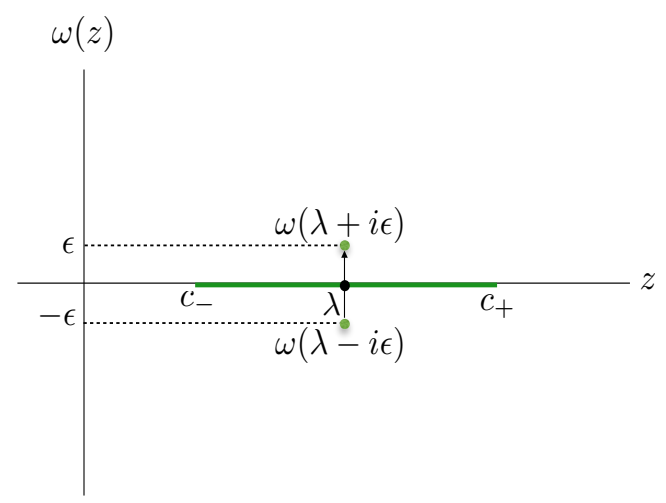

Figure 5.3: Discontinuity of the resolvent across the branch cut.

$$
\lim _{\epsilon \searrow 0} \frac{1}{\lambda-z \mp i \epsilon}=\mathcal{P}\left(\frac{1}{\lambda-z}\right) \pm i \pi \delta(\lambda-z),
$$

where $\mathcal{P}$ denotes the Cauchy principal value. Then it follows that

$$
\lim _{\epsilon \searrow 0}\left(\omega^{+}-\omega^{-}\right)=-2 \pi i \int_{\mathcal{S}} \mathrm{d} \lambda \rho(\lambda) \delta(\lambda-z)
$$

which gives rise to Stieltjes-Perron inverse formula for the density of eigenvalues

$$
\rho(\lambda)=-\frac{1}{2 \pi i} \lim _{\epsilon \searrow 0}(\omega(\lambda+i \epsilon)-\omega(\lambda-i \epsilon)) .
$$

This, in turn, can be expressed $a s^{6}$

$$
\rho(\lambda)=-\frac{1}{\pi} \lim _{\epsilon \searrow 0} \Im \omega(\lambda+i \epsilon) .
$$

Hence, if the resolvent is known, the eigenvalue density follows from the equation (5-42). However, the resolvent is still unknown. In order to find the resolvent, we substitute $\rho$ in (5-33) by its value from (5-41) which yields

$$
V^{\prime}(\lambda)=\lim _{\epsilon \searrow 0}(\omega(\lambda+i \epsilon)+\omega(\lambda-i \epsilon)), \quad \lambda \in \mathcal{S},
$$

${ }^{6}$ According to the Schwarz reflection principle [56,57], if a function $f(z)$ is analytic over some region including the real axis and is real when $z$ is real, then $f(\bar{z})=\overline{f(z)}$. Therefore, $f(z)-f(\bar{z})=2 i \Im f(z)$ and $f(z)+f(\bar{z})=2 \Re f(z)$. 
along the cut, which determines the resolvent in terms of the potential. The equations (5-41) and (5-43) define a scalar Riemann-Hilbert problem for the function $\omega(z)[56,58,59]$. In order to find the resolvent, we write it as a sum of an analytic or regular part $\omega_{r}(\lambda)$, and a singular part $\omega_{s}(\lambda)$ :

$$
\omega(\lambda)=\omega_{r}(\lambda)+\omega_{s}(\lambda)
$$

we then have

$$
\lim _{\epsilon \rightarrow 0^{+}}\left\{\left(\omega_{r}(\lambda+i \epsilon)+\omega_{s}(\lambda+i \epsilon)\right)+\left(\omega_{r}(\lambda-i \epsilon)+\omega_{s}(\lambda-i \epsilon)\right)\right\}=V^{\prime}(\lambda)
$$

or since $\omega_{r}$ is analytic,

$$
\lim _{\epsilon \searrow 0}\left\{2 \Re\left(\omega_{r}(\lambda+i \epsilon)\right)+\left(\omega_{s}(\lambda+i \epsilon)+\omega_{s}(\lambda-i \epsilon)\right)\right\}=V^{\prime}(\lambda) .
$$

It follows that the regular part of the resolvent is given $\mathrm{by}^{7}$

$$
\lim _{\epsilon \searrow 0} \Re\left(\omega_{r}(\lambda+i \epsilon)\right)=\omega_{r}(\lambda)=\frac{1}{2} V^{\prime}(\lambda),
$$

and its singular part satisfies the homogeneous equation

$$
\lim _{\epsilon \searrow 0}\left(\omega_{s}(\lambda+i \epsilon)+\omega_{s}(\lambda-i \epsilon)\right)=0
$$

The simplest one-cut solution to this equation is

$$
\sqrt{\left(\lambda-c_{-}\right)\left(\lambda-c_{+}\right)}
$$

in which $c_{-}$and $c_{+}$are the inner and outer edge of the support, respectively. The resolvent thus has the following general form under the assumption of the one-cut solution

$$
\omega(z)=\omega_{r}(z)+\omega_{s}(z)=\frac{1}{2}\left(V^{\prime}(z)-M(z) \sqrt{\left(z-c_{-}\right)\left(z-c_{+}\right)}\right),
$$

in which $M(z)$ is a polynomial whose degree is one less than that of $V^{\prime}(z)$, i.e.,

$$
\operatorname{deg} M=\operatorname{deg} V-2 .
$$

Based on the equation (5-49) and the asymptotic behavior of the resolvent, given by the equation (5-39), $M(z)$ can be determined uniquely: ${ }^{8}$

$$
M(z)=\oint_{C_{\infty}} \frac{\mathrm{d} u}{2 \pi i} \frac{M(u)}{u-z}=\oint_{C_{\infty}} \frac{\mathrm{d} u}{2 \pi i} \frac{V^{\prime}(u)}{u-z} \frac{1}{\sqrt{\left(u-c_{-}\right)\left(u-c_{+}\right)}},
$$

where $C_{\infty}$ is a counterclockwise contour enclosing the cut $\left[c_{-}, c_{+}\right]$(see Fig. 5.4). Inserting this solution for $M(z)$ into the equation (5-49), and using

${ }^{7}$ Again the first equality in (5-47) is held due to the Schwarz reflection principle.

${ }^{8}$ The first equality in (5-51) is valid for any analytic function and hence any polynomial. 


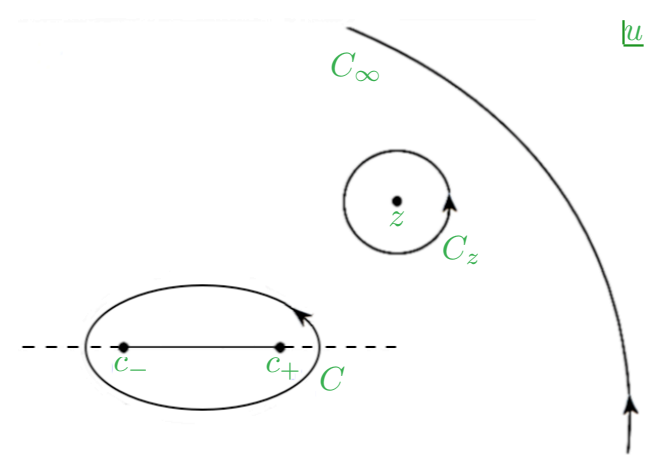

Figure 5.4: Counterclockwise contours $C, C_{z}$, and $C_{\infty}$, and the cut $\mathcal{S}=$ $\left[c_{-}, c_{+}\right]$in the complex $u$-plane.

$$
V^{\prime}(z)=\oint_{C_{z}} \frac{\mathrm{d} u}{2 \pi i} \frac{V^{\prime}(u)}{u-z}=\oint_{C_{z}} \frac{\mathrm{d} u}{2 \pi i} \frac{V^{\prime}(u)}{u-z} \sqrt{\frac{\left(z-c_{-}\right)\left(z-c_{+}\right)}{\left(u-c_{-}\right)\left(u-c_{+}\right)}},
$$

in which $C_{z}$ is a counterclockwise contour enclosing $u=z$ (see Fig. 5.4), we obtain

$$
\begin{aligned}
\omega(z)=\frac{1}{2}\left\{\oint_{C_{z}} \frac{\mathrm{d} u}{2 \pi i} \frac{V^{\prime}(u)}{u-z} \frac{1}{\sqrt{\left(u-c_{-}\right)\left(u-c_{+}\right)}}\right. \\
\left.\quad-\oint_{C_{\infty}} \frac{\mathrm{d} u}{2 \pi i} \frac{V^{\prime}(u)}{u-z} \frac{1}{\sqrt{\left(u-c_{-}\right)\left(u-c_{+}\right)}}\right\} \times \sqrt{\left(z-c_{-}\right)\left(z-c_{+}\right)}
\end{aligned}
$$

By deforming the contour $C_{\infty}$ at infinity to a counterclockwise contour $C$ which encloses the cut $\left[c_{-}, c_{+}\right]$but not $z$, and noting that ${ }_{\circlearrowleft} C_{\infty}={ }_{\circlearrowleft} C_{z}+{ }_{\circlearrowleft} C$, we deduce

$$
\omega(z)=\frac{1}{2} \oint_{C} \frac{\mathrm{d} u}{2 \pi i} \frac{V^{\prime}(u)}{z-u} \sqrt{\frac{\left(z-c_{-}\right)\left(z-c_{+}\right)}{\left(u-c_{-}\right)\left(u-c_{+}\right)}} .
$$

The solution (5-53) must have the asymptotic behavior $\omega(z) \sim 1 / z$ when $|z| \rightarrow \infty$. The asymptotic behavior of the integrand in (5-53) as a function of $z$ is as following:

$$
\frac{1}{z-u} \sqrt{\left(z-c_{-}\right)\left(z-c_{+}\right)} \underset{|z| \rightarrow \infty}{\sim} 1+\frac{u}{z}
$$

consequently, the following equations must be held

$$
\begin{aligned}
& \oint_{C} \frac{\mathrm{d} u}{2 \pi i} \frac{V^{\prime}(u)}{\sqrt{\left(u-c_{-}\right)\left(u-c_{+}\right)}}=0, \\
& \oint_{C} \frac{\mathrm{d} u}{2 \pi i} \frac{u V^{\prime}(u)}{\sqrt{\left(u-c_{-}\right)\left(u-c_{+}\right)}}=2 .
\end{aligned}
$$

These equations are enough to determine the endpoints of the cut, $c_{-}$and $c_{+}$. Equation (5-49) together with the equations (5-55) and (5-56) for the endpoints of the cut, completely solve the GUE model for polynomial potentials with one cut, in the large $N$ limit. The eigenvalue density is

$$
\rho(\lambda)=\frac{1}{2 \pi} M(\lambda) \sqrt{\left(\lambda-c_{-}\right)\left(c_{+}-\lambda\right)} .
$$


For practical purposes, we write these equations in terms of contour integrals enclosing the origin. We therefore introduce a new variable $\xi$ defined by $u=: \xi^{-1}$. The equation (5-51) then takes the form

$$
M(z)=\oint_{C_{0}} \frac{\mathrm{d} \xi}{2 \pi i} \frac{V^{\prime}(1 / \xi)}{1-z \xi} \frac{1}{\sqrt{\left(1-c_{-} \xi\right)\left(1-c_{+} \xi\right)}}
$$

in which $C_{0}$ is a counterclockwise contour which encircles $\xi=0$. In the case of equations (5-55) and (5-56), we first deform the contour $C$ to infinity and then we change the integration variable as $u=\xi^{-1}$. We then obtain the following equations:

$$
\begin{aligned}
& \oint_{C_{0}} \frac{\mathrm{d} \xi}{2 \pi i} \frac{1 / \xi V^{\prime}(1 / \xi)}{\sqrt{\left(1-c_{-} \xi\right)\left(1-c_{+} \xi\right)}}=0 \\
& \oint_{C_{0}} \frac{\mathrm{d} \xi}{2 \pi i} \frac{1 / \xi^{2} V^{\prime}(1 / \xi)}{\sqrt{\left(1-c_{-} \xi\right)\left(1-c_{+} \xi\right)}}=2 .
\end{aligned}
$$

As an Example, we apply this method to the simplest case with Gaussian potential $V(M)=\frac{1}{2} M^{2}$. We first look for the position of the endpoints. According to the equation (5-59), we must have

$$
\oint_{C_{0}} \frac{\mathrm{d} \xi}{2 \pi i} \frac{1}{\xi^{2}} \frac{1}{\sqrt{\left(1-c_{-} \xi\right)\left(1-c_{+} \xi\right)}}=0
$$

Moreover, using the Cauchy's integral formula, we have

$$
\begin{aligned}
\oint_{C_{0}} \frac{\mathrm{d} \xi}{2 \pi i} \frac{1}{\xi^{2}} \frac{1}{\sqrt{\left(1-c_{-} \xi\right)\left(1-c_{+} \xi\right)}} & =\left.\left(\frac{\mathrm{d}}{\mathrm{d} \xi} \frac{1}{\sqrt{1-\left(c_{-}+c_{+}\right) \xi+c_{-} c_{+} \xi^{2}}}\right)\right|_{\xi=0} \\
& =\frac{1}{2}\left(c_{-}+c_{+}\right)
\end{aligned}
$$

therefore $c_{-}+c_{+}=0$, in accordance with the symmetry of the potential. Taking this into account, the equation (5-60) now becomes

$$
\oint_{C_{0}} \frac{\mathrm{d} \xi}{2 \pi i} \frac{1}{\xi^{3}} \frac{1}{\sqrt{1-c_{+}^{2} \xi^{2}}}=2
$$

but

$$
\oint_{C_{0}} \frac{\mathrm{d} \xi}{2 \pi i} \frac{1}{\xi^{3}} \frac{1}{\sqrt{1-c_{+}^{2} \xi^{2}}}=\left.\frac{1}{2}\left(\frac{\mathrm{d}^{2}}{\mathrm{~d} \xi^{2}} \frac{1}{\sqrt{1-c_{+}^{2} \xi^{2}}}\right)\right|_{\xi=0}=\frac{1}{2} c_{+}^{2},
$$

therefore $c_{+}=2$. The cut is thus given by $\mathcal{S}=[-2,2]$. Now we are able to compute $M(z)$ from the equation (5-58):

$$
M(z)=\oint_{C_{0}} \frac{\mathrm{d} \xi}{2 \pi i} \frac{1}{\xi} \frac{1}{(1-z \xi) \sqrt{1-4 \xi^{2}}}=\left.\left(\frac{1}{(1-z \xi) \sqrt{1-4 \xi^{2}}}\right)\right|_{\xi=0}=1 .
$$

Next, using the equation (5-49), we obtain the resolvent for the Gaussian model:

$$
\omega(z)=\frac{1}{2}\left(z-\sqrt{z^{2}-4}\right),
$$


and eventually from the equation (5-42), we derive the density of eigenvalues for the Gaussian model:

$$
\rho(\lambda)=\frac{1}{2 \pi} \sqrt{4-\lambda^{2}}
$$

\section{2 .3}

\section{Orthogonal Polynomials Method for Unitary Ensemble with Arbitrary Potential}

The saddle point method allows a general discussion of the large $N$ limit. It is difficult, however, to calculate the subdominant orders in the $1 / N$ expansion and therefore to discuss perturbation theory to all orders. We now turn to a more powerful procedure, based on orthogonal polynomials which was first developed in [60].

The Starting point of the technique of orthogonal polynomials is the eigenvalue representation of the partition function (5-9). The idea is to disentangle the repulsive interaction between eigenvalues due to the squared Vandermonde determinant, which makes a link between random matrix theory and the classical theory of orthogonal polynomials. This method is based on one simple lemma: if we introduce a set of monic polynomials $P_{1}(\lambda), \ldots, P_{n}(\lambda)$ of order $n=0,1, \ldots, N-1$ in $\lambda$, that are orthogonal with respect to the Lebesgue measure $\mathrm{d} \mu(\lambda):=e^{-N V(\lambda)} \mathrm{d} \lambda$ on $\mathbb{R}$, namely such that,

$$
\left\langle P_{n} \mid P_{m}\right\rangle_{\mu}:=\int_{\mathbb{R}} \mathrm{d} \mu(\lambda) P_{n}(\lambda) P_{m}(\lambda)=h_{n} \delta_{n m}
$$

then by performing suitable linear combinations of preceding columns which leave the determinant unchanged, one obtains

$$
\Delta\left(\lambda_{1}, \ldots, \lambda_{N}\right) \equiv \operatorname{det}\left(\lambda_{i}^{j-1}\right)_{1 \leq i, j \leq N}=\operatorname{det}\left(P_{j-1}\left(\lambda_{i}\right)\right)_{1 \leq i, j \leq N} .
$$

Now using the Leibniz formula for determinants, we can expand (5-69) as follows

$$
\Delta\left(\lambda_{1}, \ldots, \lambda_{N}\right)=\sum_{\sigma \in S_{N}} \operatorname{sgn}(\sigma) \prod_{k=1}^{N} P_{\sigma(k)-1}\left(\lambda_{k}\right),
$$

where the sum is over permutations $\sigma$ of $N$ indices and $\operatorname{sgn}(\sigma)$ is its sign. The integrals over individual $\lambda_{i}$ 's in the eigenvalue representation of the partition function factorize, and due to orthogonality of the polynomials, the only contributions are from terms with all $P_{i}\left(\lambda_{j}\right)$ 's paired. There are $N$ ! such terms, so 


$$
\begin{aligned}
Z_{N} & =\int \prod_{k=1}^{N} \mathrm{~d} \lambda_{k} \Delta^{2}\left(\lambda_{1}, \ldots, \lambda_{N}\right) e^{-N \sum_{k=1}^{N} V\left(\lambda_{k}\right)} \\
& =\sum_{\sigma, \tau \in S_{N}} \operatorname{sgn}(\sigma) \operatorname{sgn}(\tau) \prod_{k=1}^{N} \int \mathrm{d} \mu\left(\lambda_{k}\right) P_{\sigma(k)-1}\left(\lambda_{k}\right) P_{\tau(k)-1}\left(\lambda_{k}\right) \\
& =\sum_{\sigma, \tau \in S_{N}} \delta_{\sigma \tau} \prod_{j=0}^{N-1} h_{j} \\
& =N ! \prod_{j=0}^{N-1} h_{j} .
\end{aligned}
$$

The solution of the original matrix integral is thus reduced to the problem of determining the normalization constants $h_{i}$. In order to obtain them, we notice that any orthogonal polynomials $P_{n}$ satisfy a three-term recursion relation, expressing $\lambda P_{n}(\lambda)$ in terms of $P_{n-1}(\lambda), P_{n}(\lambda)$ and $P_{n+1}(\lambda)[61]$ :

$$
\lambda P_{n}(\lambda)=s_{n} P_{n}(\lambda)+P_{n+1}(\lambda)+r_{n} P_{n-1}(\lambda) .
$$

This relation comes from the fact that $\left\langle P_{n} \mid Q\right\rangle=0$ for any polynomial $Q(\lambda)$ with $\operatorname{deg} Q<\operatorname{deg} P_{n}$. For even potentials, $\lambda P_{n}(\lambda)$ and $P_{n}(\lambda)$ have opposite parity and the term involving $P_{n}(\lambda)$ is absent $\left(s_{n}=0\right)$. Now based on the recursion relation (5-72), we demonstrate that the coefficients $r_{n}$ and $s_{n}$ can be expressed in terms of $h_{n}$ 's. From (5-72) we get

$$
\left\langle P_{n-1} \mid \lambda P_{n}\right\rangle=r_{n}\left\langle P_{n-1} \mid P_{n-1}\right\rangle=r_{n} h_{n-1} .
$$

On the other hand, in (5-72) we let $n \rightarrow n-1$. We obtain

$$
\left\langle P_{n} \mid \lambda P_{n-1}\right\rangle=r_{n}\left\langle P_{n} \mid P_{n}\right\rangle=h_{n}
$$

Left hand sides of (5-73) and (5-74) are equal, we thus obtain

$$
r_{n}=\frac{h_{n}}{h_{n-1}}, \quad n \geq 1 \text {. }
$$

The partition function is thus given by

$$
Z_{N}=N ! h_{0}^{N} \prod_{i=1}^{N} r_{i}^{N-i}
$$

In order to determine the coefficients $r_{n}$, we first introduce a set of orthonormal polynomials $\Pi_{n}(\lambda)$ as

which satisfy

$$
\Pi_{n}(\lambda):=\frac{P_{n}(\lambda)}{\sqrt{h_{n}}}=\frac{1}{\sqrt{h_{n}}} \lambda^{n}+\mathcal{O}\left(\lambda^{n-1}\right),
$$

$$
\left\langle\Pi_{n} \mid \Pi_{m}\right\rangle:=\int_{-\infty}^{\infty} \mathrm{d} \lambda e^{-N V(\lambda)} \Pi_{n}(\lambda) \Pi_{m}(\lambda)=\delta_{n m} .
$$

Next, we introduce a set of multiplication and differentiation operators which 
operate on the polynomials $\Pi_{n}$. We define the multiplication operator $B$ as

$$
B_{n m} \Pi_{m}(\lambda):=\lambda \Pi_{n}(\lambda)
$$

which following the three-term recurrence relation for the orthogonal polynomials given in (5-72) can be written as

$$
B_{n m} \Pi_{m}(\lambda)=s_{n} \Pi_{n}(\lambda)+\sqrt{r_{n+1}} \Pi_{n+1}(\lambda)+\sqrt{r_{n}} \Pi_{n-1}(\lambda),
$$

where we have used the summation convention for the matrix multiplication on the left hand side. Using the orthonormality of $\Pi_{n}$ 's, one can write the elements of the operator $B$ as

$$
B_{n m}=\left\langle\Pi_{n} \mid \lambda \Pi_{m}\right\rangle=s_{n} \delta_{n, m}+\sqrt{r_{n+1}} \delta_{n+1, m}+\sqrt{r_{n}} \delta_{n-1, m}
$$

Since $B_{n m}=\left\langle\Pi_{n} \mid \lambda \Pi_{m}\right\rangle=\left\langle\Pi_{m} \mid \lambda \Pi_{n}\right\rangle=B_{m n}$, the operator $B$ is symmetric, or in operator form

$$
B=B^{\top} .
$$

We also denote the operator of differentiation with respect to $\lambda$ by $A$ :

$$
A_{n m} \Pi_{m}(\lambda):=\partial_{\lambda} \Pi_{n}(\lambda),
$$

which in component form becomes $A_{n m}=\left\langle\Pi_{m} \mid \partial_{\lambda} \Pi_{n}\right\rangle$. Since the coefficients $h_{n}$ are independent of $\lambda$, the polynomial on the right hand side of (5-83) is at most of degree $n-1$, and hence,

$$
A_{n, n+k}=0, \quad k>-1,
$$

which means the matrix representing $A$ is strictly lower triangular.

Based on the definitions of the operators $A$ and $B$, we have

$$
(B A-A B)_{n m} \Pi_{m}=\partial_{\lambda}\left(\lambda \Pi_{n}\right)-\lambda \partial_{\lambda} \Pi_{n}=\Pi_{n}=\Pi_{m} \delta_{n m},
$$

which in operator form means that $A$ and $B$ satisfy the commutation relation

$$
[B, A]=1 .
$$

In the next step, the partition function can be determined from the so-called string equation:

$$
[B, P]=1,
$$

in which the operator $P$ is defined as

$$
P:=-\frac{N}{2}\left(V^{\prime}(B)_{+}-V^{\prime}(B)_{-}\right) .
$$

Here + and - refer to the upper and lower triangular parts of the matrix, respectively. The diagonal part of $P$ vanishes and since $B$ is symmetric, obviously $P$ 
is antisymmetric. Because $P$ is expressed only in terms of powers of $B$, which is tridiagonal, $P$ has only a finite number of non-zero off-diagonals.

We are particularly interested in computing the eigenvalue distribution. In fact, in the large $N$ limit, the coefficients of the recursion relation become continuous variables of the parameter $0 \leq \xi=\frac{n}{N} \leq 1$, i.e., $r_{n} \rightarrow r(\xi)$ and $s_{n} \rightarrow s(\xi)$. Then it can be demonstrated that [47]:

$$
\rho(\lambda)=\frac{1}{\pi} \int_{0}^{1} \mathrm{~d} \xi \frac{\theta(f(\xi ; \lambda))}{\sqrt{f(\xi ; \lambda)}}
$$

where $\theta(\cdot)$ is the Heaviside unit step function and $f(\xi ; \lambda)=4 r(\xi)-(\lambda+s(\xi))^{2}$. It follows from this equation that $\rho(\lambda)$ is supported on the interval $[-2 \sqrt{r(1)}-$ $s(1), 2 \sqrt{r(1)}-s(1)]$.

\subsection{4}

\section{Results of Orthogonal Polynomials Method for GUE}

The case of Gaussian potential can be handled easily. In fact, we already know that Hermite polynomials defined as

$$
H_{n}(\lambda):=(-1)^{n} e^{\lambda^{2} / 2} \frac{\mathrm{d}^{n}}{\mathrm{~d} \lambda^{n}} e^{-\lambda^{2} / 2}, \quad n=0, \ldots, N-1,
$$

are orthogonal with respect to the standard normal probability density function, i.e.,

$$
\int_{\mathbb{R}} \mathrm{d} \lambda e^{-\lambda^{2} / 2} H_{n}(\lambda) H_{m}(\lambda)=\sqrt{2 \pi} n ! \delta_{n m} .
$$

By changing the integration variable as $\lambda=: \sqrt{N} \lambda^{\prime}$, we obtain

$$
\int_{\mathbb{R}} \mathrm{d} \lambda^{\prime} e^{-N \lambda^{\prime 2} / 2} H_{n}\left(\sqrt{N} \lambda^{\prime}\right) H_{m}\left(\sqrt{N} \lambda^{\prime}\right)=\sqrt{2 \pi / N} n ! \delta_{n m}
$$

which immediately gives

$$
h_{n}=\sqrt{2 \pi / N} n !, \quad P_{n}(\lambda)=\left(\frac{N}{2 \pi}\right)^{1 / 4} \frac{1}{\sqrt{n !}} H_{n}(\sqrt{N} \lambda) .
$$

Furthermore, one can obtain the string equation for Gaussian potential with $V(M)=M^{2}$, and thereby the coefficients $r_{n}$ and $s_{n}$. In fact, in this case one has

$$
P_{n m}^{\text {Gauss }}=-N\left(\sqrt{r_{n+1}} \delta_{n+1, m}-\sqrt{r_{n}} \delta_{n-1, m}\right) .
$$

Recursion relations for $r_{n}$ can be obtained from the string equation (8-35) by considering the diagonals, i.e., we have the recursion relations

$$
[B, P]_{n, n}-1=\left(\sqrt{r_{n+1}} V^{\prime}(B)_{n+1, n}-\frac{n+1}{N}\right)-\left(\sqrt{r_{n}} V^{\prime}(B)_{n, n-1}-\frac{n}{N}\right)=0
$$


and

$$
[B, P]_{n, n-1}=V^{\prime}(B)_{n, n}-V^{\prime}(B)_{n-1, n-1}=0,
$$

which are, in turn, implied by the integrated form of the string equation [53]:

$$
\begin{aligned}
& \sqrt{r_{n}} V^{\prime}(B)_{n, n-1}=\frac{n}{N}, \quad V^{\prime}(B)_{n, n}=0 . \\
& \rho(\lambda)=\frac{1}{\pi} \int_{0}^{1} \mathrm{~d} \xi \frac{\theta\left(4 \xi-\lambda^{2}\right)}{\sqrt{4 \xi-\lambda^{2}}}=\frac{1}{2 \pi} \sqrt{4-\lambda^{2}}
\end{aligned}
$$

which is in agreement with the previous result obtained by saddle point method. 


\section{6 \\ Ginibre Ensemble}

Pure mathematics is, in its way, the poetry of logical ideas.

A. Einstein

Hermitian RMT is of special importance in physics, because of the Hermiticity of operators associated with observables in quantum mechanics. However, nonHermitian random matrices have also attracted considerable attention, in particular because they can be used as models for dissipative or open physical systems [62-64].

The study of eigenvalue statistics in the complex plane was initiated by Ginibre [65]. Statistics of complex eigenvalues have many interesting applications in modeling of a wide range of physical phenomena. They appear in the studies of quantum chromodynamics at finite chemical potential [66], dissipative quantum maps with eigenvalues having imaginary parts due to dissipation [67], fractional quantum-Hall effect [68], and many other areas.

In this chapter, we show how the resolvent formalism, introduced in Ch. 5 can be again employed to obtain the eigenvalue density of non-Hermitian Gaussian model. This is done by implementing a diagrammatic method, first for a Hermitian model, followed by its generalisation for a non-Hermitian model.

\section{1}

\section{Resolvent Formalism}

Ginibre ensembles with non-Gaussian weight are typically difficult to deal with due to the lack of symmetry. We consider the simplest case, the so-called complex Ginibre ensemble, with $V\left(X, X^{\dagger}\right)=X X^{\dagger}$ and the probability measure

$$
\mathrm{d} \mu(X)=\frac{1}{Z_{N}} e^{-N \operatorname{Tr}\left(X X^{\dagger}\right)} \mathrm{d} X,
$$

in which the integration measure is the following Lebesgue measure:

$$
\mathrm{d} X=\prod_{i, j=1}^{N} \mathrm{~d}\left(\Re X_{i j}\right) \mathrm{d}\left(\Im X_{i j}\right)
$$


We shall follow here the standard strategy of calculating the eigenvalue density of a random matrix by first calculating the resolvent (or Green's function) $\omega(z, \bar{z})$ and then using an exact relation between the eigenvalue density and the resolvent. The crucial difference between the Hermitian and non-Hermitian case comes from the fact that in non-Hermitian random matrix models, eigenvalues do not lie on the real axis. In the large $N$ limit, they may accumulate in two-dimensional domains in the complex plane and the corresponding eigenvalue density

$$
\rho(z, \bar{z}):=\lim _{N \rightarrow \infty} \mathbb{E}\left[\frac{1}{N} \sum_{i=1}^{N} \delta^{(2)}\left(z-\lambda_{i}\right)\right],
$$

where $\lambda_{i}$ 's are eigenvalues of $X$ and $\mathbb{E}[\cdot]$ denotes averaging over a given ensemble of $N \times N$ random non-Hermitian matrices generated by (6-1), may become a continuous function with an extended support in the complex plane. If one wants to apply the resolvent formalism for (6-3), one has to find a representation of the two-dimensional delta function. A natural candidate is ${ }^{1}$

$$
\delta^{(2)}(z-\lambda)=\frac{1}{\pi} \lim _{\epsilon \rightarrow 0} \frac{\epsilon^{2}}{\left(|z-\lambda|^{2}+\epsilon^{2}\right)^{2}}=\frac{1}{\pi} \lim _{\epsilon \rightarrow 0} \frac{\partial}{\partial \bar{z}}\left[\frac{\bar{z}-\bar{\lambda}}{|z-\lambda|^{2}+\epsilon^{2}}\right] .
$$

Using this representation, one can write

$$
\rho(z, \bar{z})=\lim _{\epsilon \rightarrow 0} \lim _{N \rightarrow \infty} \mathbb{E}\left[\frac{1}{N} \sum_{i=1}^{N} \frac{\epsilon^{2}}{\left(\left|z-\lambda_{i}\right|^{2}+\epsilon^{2}\right)^{2}}\right]
$$

or

$$
\rho(z, \bar{z})=\frac{1}{\pi} \frac{\partial^{2} \zeta(z, \bar{z})}{\partial z \partial \bar{z}}
$$

where

$$
\zeta(z, \bar{z})=\lim _{\epsilon \rightarrow 0} \lim _{N \rightarrow \infty} \mathbb{E}\left[\frac{1}{N} \sum_{i=1}^{N} \ln \left(\left|z-\lambda_{i}\right|^{2}+\epsilon^{2}\right)\right],
$$

or equivalently

$$
\zeta(z, \bar{z})=\lim _{\epsilon \rightarrow 0} \lim _{N \rightarrow \infty} \mathbb{E}\left[\frac{1}{N} \operatorname{Tr} \ln \left((z \mathbb{1}-X)\left(\bar{z} \mathbb{1}-X^{\dagger}\right)+\epsilon^{2} \mathbb{1}\right)\right] .
$$

One can interpret (6-5) (or equivalently (6-6)), as a Poisson equation for electrostatics $^{2}$ where $\rho(z, \bar{z})$ is a two dimensional charge distribution, and $\zeta(z, \bar{z})$ is a elec-

${ }^{1}$ The principal value representation of the Dirac delta function in the real domain is

$$
\delta(x)=-\frac{1}{\pi} \lim _{\epsilon \searrow 0} \frac{1}{x+i \epsilon}=\frac{1}{\pi} \lim _{\epsilon \searrow 0} \frac{\epsilon}{x^{2}+\epsilon^{2}},
$$

which can be verified by applying the Sokhotski-Weierstrauss theorem (5-40) to the function $f(x)=\frac{1}{x}$. The representation (6-4) is a natural extension to two dimensions which satisfies the desired properties of the Dirac delta function.

${ }^{2}$ We recall that if $z=x+i y$, then

$$
\frac{\partial}{\partial z}=\frac{1}{2}\left(\frac{\partial}{\partial x}-i \frac{\partial}{\partial y}\right), \quad \frac{\partial}{\partial \bar{z}}=\frac{1}{2}\left(\frac{\partial}{\partial x}+i \frac{\partial}{\partial y}\right) .
$$


trostatic potential $[63,65,69-72]$. One can further exploit the electrostatic analogy by introducing the corresponding electric field, which is indeed the resolvent:

$$
\begin{aligned}
\omega(z, \bar{z}) \equiv \frac{\partial \zeta}{\partial z} & =\lim _{\epsilon \rightarrow 0} \lim _{N \rightarrow \infty} \mathbb{E}\left[\frac{1}{N} \sum_{i=1}^{N} \frac{\bar{z}-\bar{\lambda}_{i}}{\left|z-\lambda_{i}\right|^{2}+\epsilon^{2}}\right] \\
& =\lim _{\epsilon \rightarrow 0} \lim _{N \rightarrow \infty} \mathbb{E}\left[\frac{1}{N} \operatorname{Tr} \frac{\bar{z} \mathbb{1}-X^{\dagger}}{\left(\bar{z} \mathbb{1}-X^{\dagger}\right)(z \mathbb{1}-X)+\epsilon^{2} \mathbb{1}}\right]
\end{aligned}
$$

$\zeta$ is a real-valued function on the complex plane, so it is a scalar field from the point of view of two dimensional electrostatics, while $\omega$ is a complex function and vector field. Moreover, the complex form of the Stieltjes-Perron inversion formula (see (5-42)) lets us to write the Poisson equation as a Gauss law in two dimensions [69-73]:

or

$$
\rho(z, \bar{z})=\frac{1}{\pi} \frac{\partial}{\partial \bar{z}}\left\{\lim _{\epsilon \rightarrow 0} \lim _{N \rightarrow \infty} \mathbb{E}\left[\frac{1}{N} \sum_{i=1}^{N} \frac{\bar{z}-\bar{\lambda}_{i}}{\epsilon^{2}+\left|z-\lambda_{i}\right|^{2}}\right]\right\}
$$

$$
\rho(z, \bar{z})=\frac{1}{\pi} \frac{\partial \omega(z, \bar{z})}{\partial \bar{z}}
$$

In the large $N$ limit, when the eigenvalues $\lambda_{i}$ of the random matrix concentrate in a certain domain $D \subset \mathbb{C}$ of the complex plane, the resolvent $\omega(z, \bar{z})$ is no longer holomorphic. Actually, as one can deduce from (6-11), the eigenvalue distribution is related to the non-holomorphic behavior of the resolvent, i.e., the eigenvalue distribution is non-zero, exactly where the resolvent ceases to be analytic $\left(\frac{\partial \omega}{\partial \bar{z}} \neq 0\right){ }^{3}$

Before proceeding further, we remark a slight difference between our problem and electrostatics. In electrostatics, one usually applies the Gauss law to determine the electric field for a given charge density. In our problem, we proceed in the opposite direction: first, we calculate the resolvent or Green's function (electric field) and then we use it to determine the eigenvalue density.

Note that the resolvent (6-9) is a complicated object which does not resemble its Hermitian counterpart (5-34). In particular, in contrast to the Hermitian case, we can not just apply the geometric series expansion as the Hermitian case (see (5-35)). We can however, use a trick invented in [74], which allows us to apply the geometric series expansion, but for a larger $2 N \times 2 N$ matrix $\Omega$ with four $N \times N$ blocks:

Then, the Poisson equation $\nabla^{2} \phi(x, y)=-\rho(x, y)$ can be written as $4 \frac{\partial^{2} \phi(z, \bar{z})}{\partial z \partial \bar{z}}=-\rho(z, \bar{z})$.

${ }^{3}$ We recall that a function $f$ is analytic in a domain $D$, if $\frac{\partial f}{\partial \bar{z}}=0, \forall z \in D$. 


$$
\Omega \equiv\left(\begin{array}{ll}
\Omega_{z z} & \Omega_{z \bar{z}} \\
\Omega_{\bar{z} z} & \Omega_{\overline{z z}}
\end{array}\right):=\lim _{\epsilon \rightarrow 0} \lim _{N \rightarrow \infty} \mathbb{E}\left[\frac{1}{N} \operatorname{Tr}_{b}\left(\begin{array}{cc}
z \mathbb{1}-X & i \epsilon \mathbb{1} \\
i \epsilon \mathbb{1} & \bar{z} \mathbb{1}-X^{\dagger}
\end{array}\right)^{-1}\right] .
$$

in which the subscripts $z z, z \bar{z}, \bar{z} z$, and $\overline{z z}$ refer to the position of the $N \times N$ blocks in the corresponding $2 N \times 2 N$ matrix and we have introduced the block-trace operation

$$
\operatorname{Tr}_{b}\left(\begin{array}{ll}
A & B \\
C & D
\end{array}\right)_{2 N \times 2 N}:=\left(\begin{array}{cc}
\operatorname{Tr} A & \operatorname{Tr} B \\
\operatorname{Tr} C & \operatorname{Tr} D
\end{array}\right)_{2 \times 2},
$$

which reduces $2 N \times 2 N$ matrices to $2 \times 2$ ones. The elements of $\Omega$ are given by

$$
\begin{aligned}
& \Omega_{z z}(z, \bar{z})=\lim _{\epsilon \rightarrow 0} \lim _{N \rightarrow \infty} \mathbb{E}\left[\frac{1}{N} \operatorname{Tr} \frac{\bar{z} \mathbb{1}-X^{\dagger}}{\left(\bar{z} \mathbb{1}-X^{\dagger}\right)(z \mathbb{1}-X)+\epsilon^{2} \mathbb{1}}\right] \\
& \Omega_{z \bar{z}}(z, \bar{z})=\lim _{\epsilon \rightarrow 0} \lim _{N \rightarrow \infty} \mathbb{E}\left[\frac{1}{N} \operatorname{Tr} \frac{-i \epsilon \mathbb{1}}{\left(\bar{z} \mathbb{1}-X^{\dagger}\right)(z \mathbb{1}-X)+\epsilon^{2} \mathbb{1}}\right] \\
& \Omega_{\bar{z} z}(z, \bar{z})=\lim _{\epsilon \rightarrow 0} \lim _{N \rightarrow \infty} \mathbb{E}\left[\frac{1}{N} \operatorname{Tr} \frac{-i \epsilon \mathbb{1}}{\left(\bar{z} \mathbb{1}-X^{\dagger}\right)(z \mathbb{1}-X)+\epsilon^{2} \mathbb{1}}\right] \\
& \Omega_{\overline{z z}}(z, \bar{z})=\lim _{\epsilon \rightarrow 0} \lim _{N \rightarrow \infty} \mathbb{E}\left[\frac{1}{N} \operatorname{Tr} \frac{z \mathbb{1}-X}{\left(\bar{z} \mathbb{1}-X^{\dagger}\right)(z \mathbb{1}-X)+\epsilon^{2} \mathbb{1}}\right] .
\end{aligned}
$$

By comparing (6-9) and (6-14), one observes that

$$
\omega(z, \bar{z})=\Omega_{z z}(z, \bar{z}) .
$$

In order to calculate the Green's function (6-9) or the matrix $\Omega$ in (6-12), one has to perform a double limit. It is important to take the limits in the correct order: first $N$ tends to infinity and after $\epsilon$ goes to zero, since if one took this limit in the opposite order by first setting $\epsilon=0$ for finite $N$, the expression in the brackets in (6-9) has isolated poles on the complex plane. However, in the limit $N \rightarrow \infty$, the poles merge into a cut. One can not then make an analytic continuation of the function from holomorphic to non-holomorphic region, as it is done when calculating $\Omega$ by diagrammatic method which utilizes $O\left(z^{-1}\right)$ expansion. A small $\epsilon>0$ is necessary to make $\Omega$ analytic everywhere. If one first took the limit $\epsilon \rightarrow 0$, and then the limit $N \rightarrow \infty$, the matrix $\Omega$ would become block-diagonal: $\Omega_{z z}=\mathbb{E}\left[(z \mathbb{1}-X)^{-1} / N\right], \Omega_{\overline{z z}}^{\dagger}=\mathbb{E}\left[\left(\bar{z} \mathbb{1}-X^{\dagger}\right)^{-1} / N\right]$ and $\Omega_{z \bar{z}}=\Omega_{\bar{z} z}=0$, which is in contrast to (6-14).

Finally, whenever we apply generating functions for planar diagrams, we can automatically take the limit $\epsilon \rightarrow 0$, which trivially amounts to setting $\epsilon=0$, since the limit $N \rightarrow \infty$ has already been taken by the planar approximation used to write relations between generating functions for planar diagrams. 


\section{2}

\section{Diagrammatic Method for Hermitian Random Matrices}

In the Hermitian case, there are several ways of calculating resolvent, like saddlepoint method which we already discussed, and loop equations [75, 76]. Here, we follow the diagrammatic approach. The starting point of the analysis is the expression allowing for the reconstruction of the Green's function from all the moments $\mathbb{E}\left[\operatorname{Tr} M^{n}\right]$ :

where

$$
\omega(z)=\mathbb{E}\left[\frac{1}{N} \operatorname{Tr} \frac{1}{Z-M}\right]=\frac{1}{N} \operatorname{Tr} \Omega(z),
$$

$$
\Omega(z)=Z^{-1}+\mathbb{E}\left[Z^{-1} M Z^{-1} M Z^{-1}\right]+\mathbb{E}\left[Z^{-1} M Z^{-1} M Z^{-1} M Z^{-1} M Z^{-1}\right]+\cdots
$$

in which $Z:=z \mathbb{1}$ is independent of $M$ 's and can be pulled out of the averaging brackets. What remains are correlation functions of the type $\mathbb{E}\left[M_{i_{1} i_{2}} \cdots M_{i_{2 n-1} i_{2 n}}\right]$ which using the Wick theorem can be expressed as products of two-point functions (propagators). We will use the diagrammatic method to evaluate efficiently the sum of the moments on the right hand side of (6-17). We note, however, that in general this series expansion is convergent only in a neighborhood of $z=\infty$. So the results of the diagrammatic calculation apply directly only there. For Hermitian random matrices this does not pose a problem. Since the eigenvalues lie only on some intervals on the real axis, the Green's function is a holomorphic function of $z$ on the complex plane, except for the cuts on the real axis. Therefore, we can reconstruct the Green's function everywhere by analytical continuation.

For illustration, we consider now the well-known case of a random Hermitian ensemble with Gaussian distribution. We introduce a generating function with a matrix source $J$ :

$$
Z(J):=\int \mathrm{d} M e^{-\frac{N}{2} \operatorname{Tr} M^{2}+\operatorname{Tr} M . J}
$$

in which $M=M^{\dagger}$, and

$$
\mathrm{d} M=\prod_{i=1}^{N} \mathrm{~d} M_{i i} \prod_{1 \leq i<j \leq N} \mathrm{~d}\left(\Re M_{i j}\right) \mathrm{d}\left(\Im M_{i j}\right) .
$$

All the moments follow directly from $Z(J)$ through the relation

$$
\mathbb{E}\left[\operatorname{Tr} M^{n}\right]=\left.\frac{1}{Z(0)} \operatorname{Tr}\left(\frac{\partial}{\partial J}\right)^{n} Z(J)\right|_{J=0},
$$

and are straightforward to calculate, since in this case the partition function reads $Z(J)=e^{\frac{1}{2 N} \operatorname{Tr} J^{2}}$. The propagator thus reads 


$$
\left(Z^{-1}\right)_{a b}=\stackrel{\bullet}{\bullet} \quad \dot{b}
$$

Figure 6.1: $\left(Z^{-1}\right)_{a b}$ depicted as a line between $a$ and $b$.

$$
\mathbb{E}\left[M_{a b} M_{c d}\right]=\frac{\partial^{2} Z(J)}{\partial J_{b a} \partial J_{d c}}=\frac{1}{N} \delta_{a d} \delta_{b c}
$$

The key idea in the diagrammatic approach is to associate to the expressions for the moments, like (6-21), a graphical representation following a simple set of rules. The power of this approach is that it enables to perform a resummation of the whole power series (6-17) by identification of the structure of the relevant graphs. This observation allows us to represent (6-17) as a sum over Feynman diagrams [77]. As depicted in Fig. 6.1, the term $\left(Z^{-1}\right)_{a b}$ is represented by a horizontal line, joining the indices $a$ and $b$. Each propagator (6-21) is depicted as a double arc joining two pairs of matrix indices (Fig. 6.2). In order to calculate $\Omega_{a b}$, one has to sum up contributions of all connected diagrams with two external points $a$ and $b$ (Fig. 6.3). For finite $N$, this is a formidable task, since there are infinitely many diagrams. The problem, however, simplifies significantly in the limit $N \rightarrow \infty$, because all nonplanar diagrams can be neglected $[58,78]$. It turns out that all planar diagrams can be summed up using a trick, known as Dyson-Schwinger equations. In this method, the resummation (6-17) is done by introducing the self-energy $\Sigma$ as a generating function for all one-line irreducible diagrams (Fig. 6.4). $\Sigma_{a b}$ generates all one-line irreducible diagrams with vertices $a$ and $b . \Omega$ and $\Sigma$ are related to each other through the matrix equation (Fig. 6.5)

$$
\Omega(z)=\frac{1}{z-\Sigma(z)}
$$

which can be interpreted as the definition of $\Sigma$. However, one can write another independent equation for $\Omega$ and $\Sigma$. It follows from observation that any one-line irreducible diagram can be obtained from a diagram of $\Omega$ by adding an arc to it (Fig. 6.6), i.e.,

$$
\Sigma(z)=\Omega(z) .
$$

Combining (6-22) and (6-23) gives immediately

$$
\omega_{ \pm}(z)=\frac{1}{2}\left(z \pm \sqrt{z^{2}-4}\right) .
$$

Only the $\omega_{-}$has the proper asymptotic behaviour $\omega(z) \underset{|z| \rightarrow \infty}{\sim} \frac{1}{z}$ (see (5-39)). From the discontinuity equation (5-42), one can recover the Wigner semi-circle law for the distribution of the eigenvalues:

$$
\rho(\lambda)=\frac{1}{2 \pi} \sqrt{4-\lambda^{2}} .
$$




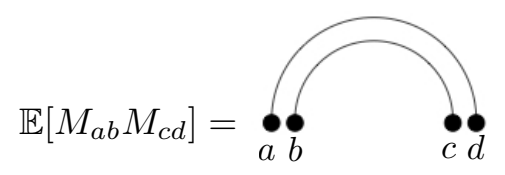

Figure 6.2: The propagator $\mathbb{E}\left[M_{a b} M_{c d}\right]$ represented as a double arc, joining $a$ with $d$, and $b$ with $c$, respectively.

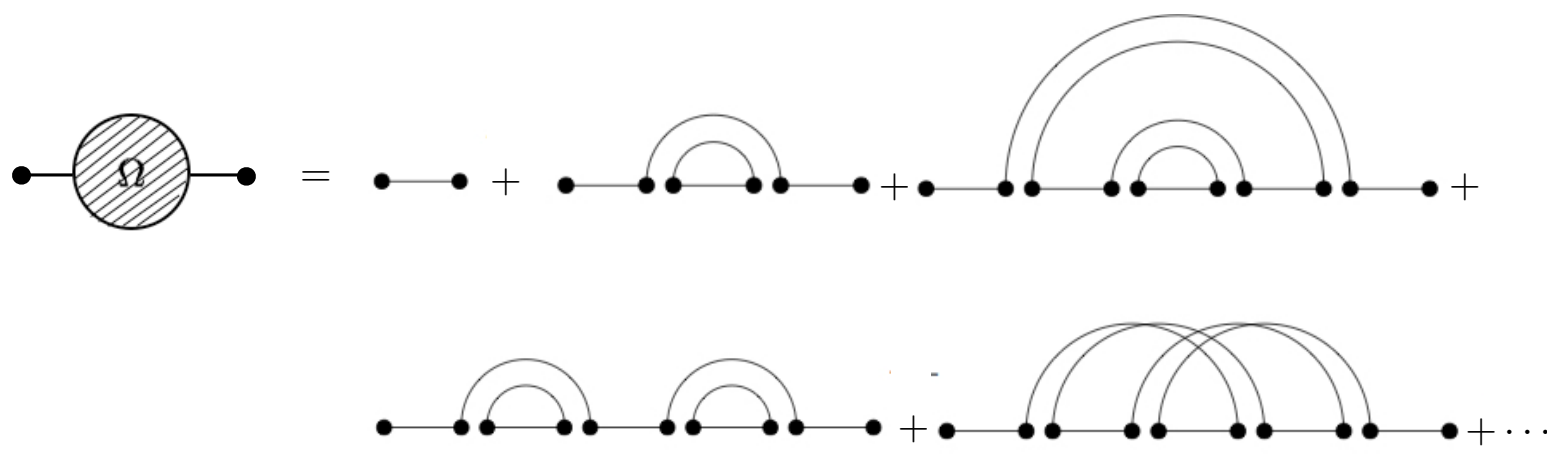

Figure 6.3: Diagrammatical representation of Eq. (6-17).

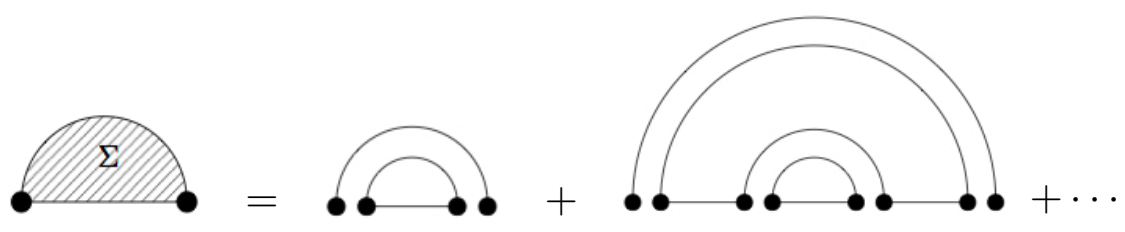

Figure 6.4: Diagrammatical representation of self-energy $\Sigma$.

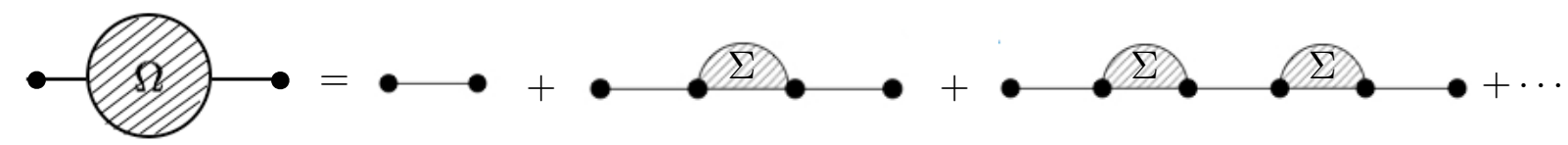

Figure 6.5: Diagrammatical representation of the first Dyson-Schwinger equation.

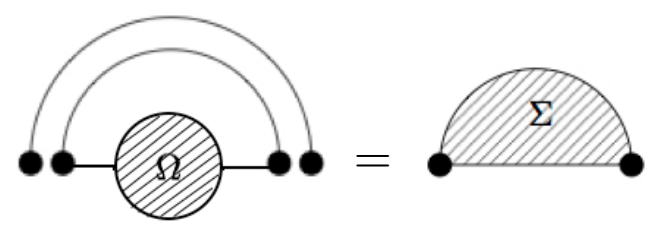

Figure 6.6: Diagrammatical representation of the second Dyson-Schwinger equation. 


\section{3}

\section{Diagrammatic Method for Non-Hermitian Random Matrices}

Before applying the diagrammatic method to non-Hermitian random matrices, we try to understand why the direct series expansion of the resolvent does not work when it is applied to the simplest non-Hermitian ensemble, namely Ginibre-Girko ensemble $[65,72]$ with non-Hermitian matrices $X$, and the measure (6-1).

One can easily see that all moments $\mathbb{E}\left[\operatorname{Tr} X^{n}\right]$ are zero for $n=1,2, \ldots$ It follows from the expansion (6-17) that $\omega(z, \bar{z})=1 / z$ for all $z \in \mathbb{C}$. (this follows diagrammatically from the fact that the propagator $\mathbb{E}\left[X_{a b} X_{c d}\right]$ vanishes, and hence $\Sigma=0)$. The correct answer is, however, different, and as we shall see, one has $\omega(z, \bar{z})=1 / z$ only for $|z|>1$. For $|z|<1$, the resolvent is non-holomorphic and is given by $\omega(z, \bar{z})=\bar{z}$. This failure is due to the fact that the resolvent has poles which are scattered in the complex $z$-plane. In the limit $N \rightarrow \infty$, the poles accumulate in two dimensional regions in the complex plane, over which the resolvent is no longer holomorphic (compare with the case of Hermitian random matrices in which the real poles merge into a one dimensional cut in on the real axis). Therefore, the resolvent can not be continued analytically to the interesting region where $\partial \omega / \partial \bar{z} \neq 0$ with a finite eigenvalue distribution.

It then follows that we must look for a generalized method which works for small $z$ too. Furthermore, we would like to implement the flexible diagrammatical method and express the desired quantities in terms of some generalized moments.

Now, we calculate the resolvent in the case of non-Hermitian random matrices with the probability measure

$$
\mathrm{d} \mu(X) \propto e^{-N \operatorname{Tr}\left(X X^{\dagger}\right)} \prod_{i, j=1}^{N} \mathrm{~d}\left(\Re X_{i j}\right) \mathrm{d}\left(\Im X_{i j}\right) .
$$

One can calculate the propagators easily:

$$
\begin{aligned}
\mathbb{E}\left[X_{a b} X_{c d}\right] & =\mathbb{E}\left[X_{a b}^{\dagger} X_{c d}^{\dagger}\right]=0 \\
\mathbb{E}\left[X_{a b} X_{c d}^{\dagger}\right] & =\mathbb{E}\left[X_{a b}^{\dagger} X_{c d}\right]=\frac{1}{N} \delta_{a d} \delta_{b c}
\end{aligned}
$$

As before, we define a $2 N \times 2 N$ matrix

$$
\mathcal{X}:=\left(\begin{array}{ll}
\mathcal{X}_{z z} & \mathcal{X}_{z \bar{z}} \\
\mathcal{X}_{\bar{z} z} & \mathcal{X}_{\overline{z z}}
\end{array}\right) \equiv\left(\begin{array}{cc}
X & 0 \\
0 & X^{\dagger}
\end{array}\right) .
$$

Analogously, the resolvent $\Omega$, the self-energy $\Sigma$, and the matrix $Z$ are $2 N \times 2 N$ 
matrices:

$$
\Omega=\left(\begin{array}{cc}
\Omega_{z z} & \Omega_{z \bar{z}} \\
\Omega_{\bar{z} z} & \Omega_{\overline{z z}}
\end{array}\right), \quad \Sigma=\left(\begin{array}{cc}
\Sigma_{z z} & \Sigma_{z \bar{z}} \\
\Sigma_{\bar{z} z} & \Sigma_{\overline{z z}}
\end{array}\right), \quad Z=\left(\begin{array}{cc}
Z_{z z} & Z_{z \bar{z}} \\
Z_{\bar{z} z} & Z_{\bar{z} \bar{z}}
\end{array}\right) .
$$

Then, following equation is held:

$$
\left(\begin{array}{ll}
\Omega_{z z} & \Omega_{z \bar{z}} \\
\Omega_{\bar{z} z} & \Omega_{\overline{z z}}
\end{array}\right)=\left(\begin{array}{ll}
Z_{z z}-\Sigma_{z z} & Z_{z \bar{z}}-\Sigma_{z \bar{z}} \\
Z_{\bar{z} z}-\Sigma_{\bar{z} z} & Z_{\overline{z z}}-\Sigma_{\overline{z z}}
\end{array}\right) .
$$

The second equation, which is analogue of (6-22), can be derived using the propagators in (6-27):

$$
\left(\begin{array}{cc}
\Sigma_{z z} & \Sigma_{z \bar{z}} \\
\Sigma_{\bar{z} z} & \Sigma_{\overline{z z}}
\end{array}\right)=\left(\begin{array}{cc}
\Omega_{z z} \cdot 0 & \Omega_{z \bar{z}} \\
\Omega_{\bar{z} z} & \Omega_{\overline{z z}} \cdot 0
\end{array}\right)=\left(\begin{array}{cc}
0 & \Omega_{z \bar{z}} \\
\Omega_{\bar{z} z} & 0
\end{array}\right)
$$

The diagonal entries are zero, due to vanishing first two propagators in (6-27). The matrix equations (6-30) and (6-31) completely solve the problem. Inserting (6-31) to (6-30) yields:

$$
\left(\begin{array}{ll}
\Omega_{z z} & \Omega_{z \bar{z}} \\
\Omega_{\bar{z} z} & \Omega_{\overline{z z}}
\end{array}\right)=\frac{1}{|z|^{2}-\Omega_{z \bar{z}} \Omega_{\bar{z} z}}\left(\begin{array}{cc}
\bar{z} & \Omega_{z \bar{z}} \\
\Omega_{\bar{z} z} & z
\end{array}\right) .
$$

Looking at off-diagonal equations, we see that there are two solutions: the first one corresponds to $\Omega_{z \bar{z}}=\Omega_{\bar{z} z}=0$, which gives the holomorphic solution $\omega(z, \bar{z})=$ $1 / z$, and is valid for large $|z|$; while the second solution is non-holomorphic and corresponds to $|z|^{2}-\Omega_{z \bar{z}} \Omega_{\bar{z} z}=1$, and results in $\omega(z, \bar{z})=\bar{z}$. Both solutions match at $|z|=1$ and we can write the complete result as

$$
\omega(z, \bar{z})= \begin{cases}\bar{z}, & |z| \leq 1 \\ \frac{1}{z}, & |z|>1\end{cases}
$$

which results in celebrated Ginibre-Girko distribution, also called as circular law: ${ }^{4}$

$$
\rho(z, \bar{z})=\frac{\partial \omega(z, \bar{z})}{\partial \bar{z}}= \begin{cases}\frac{1}{\pi}, & |z| \leq 1 \\ 0, & |z|>1\end{cases}
$$

The complex eigenvalues of the Ginibre ensemble are therefore uniformly distributed on the unit disc (see Fig. 6.7).

Briefly, using diagrammatic method, one can obtain a set of algebraic equations for $\Omega$ and $\Sigma$ in the large $N$ limit. One can set $\epsilon=0$ in the equations, since they are already obtained in the limit $N \rightarrow \infty$.

\footnotetext{
${ }^{4}$ We recall that for $z=x+i y, \frac{\partial}{\partial \bar{z}}\left(\frac{1}{z}\right)=\frac{\partial}{\partial z}\left(\frac{1}{\bar{z}}\right)=\pi \delta(x) \delta(y)=\pi \delta^{(2)}(z)=0, \forall z \neq 0$.
} 


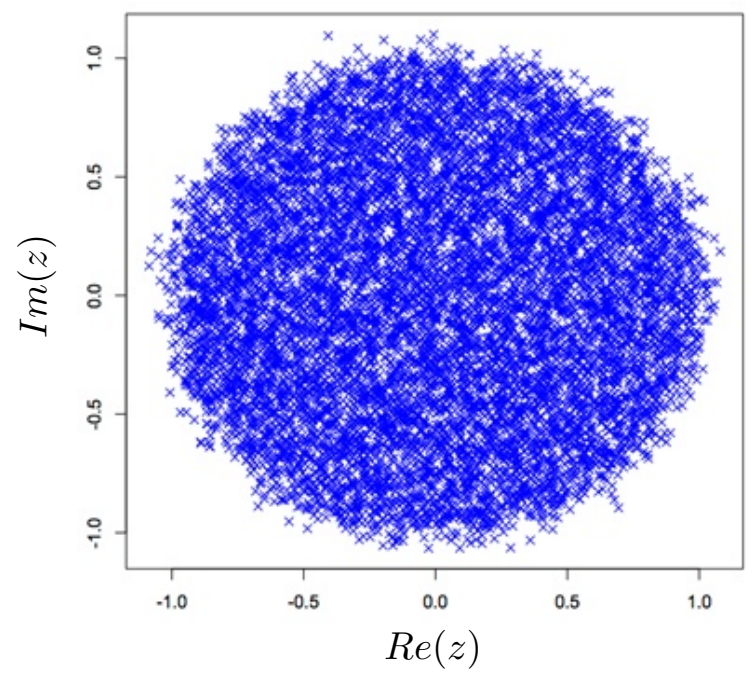

Figure 6.7: Circular law for the Ginibre ensemble. 


\section{Random Normal Matrix Ensembles}

While due to the absence of a simplifying symmetry, the study of non-Hermitian random matrices is, in general, a difficult task, normal random matrices are interesting models to consider, since they offer more probability measures amenable to analytical investigations. Specially, the method of orthogonal polynomials which is not applicable to the non-Hermitian matrix models, is a useful technique in the study of normal random matrices.

By definition, a normal matrix is a square matrix which commutes with its Hermitian adjoint. In this chapter, we consider ensembles of normal matrices with polynomial potentials. Such ensembles are exactly solvable, because a normal matrix and its adjoint are diagonalizable simultaneously by a unitary transformation.

Normal random matrix ensembles were first introduced in [79] in order to study fractional quantum-Hall effect. In fact, as we already observed in Ch. 5 , it is wellknown that wave functions describing the one-dimensional repulsive Coulomb gas of particles can be constructed from the orthogonal polynomials of the Hermitian matrix models. Analogously, the wave functions describing the two dimensional case can be constructed from the orthogonal polynomials of the corresponding normal matrix model.

\section{1}

\section{Eigenvalue Representation of the Partition Function}

We consider an ensemble of $N \times N$ random normal matrices $M$ which satisfy the constraint

$$
\left[M, M^{\dagger}\right]=0
$$

with the following probability measure:

$$
\mathbb{P}(M) \mathrm{d} M=\frac{1}{Z_{N}} e^{-N \operatorname{Tr} V(M)} \mathrm{d} M,
$$

in which the partition function of the model is

$$
Z_{N}=\int_{\mathcal{N}_{N}} \mathrm{~d} M e^{-N \operatorname{Tr} V(M)}
$$


Here, $\mathcal{N}_{N}$ denotes the set of all $N \times N$ normal random matrices and the integration measure is given by

$$
\mathrm{d} M=\prod_{i, j=1}^{N} \mathrm{~d}\left(\Re M_{i j}\right) \mathrm{d}\left(\Im M_{i j}\right) .
$$

Since $M$ is a normal matrix, there exists a unitary matrix $U$ which simultaneously diagonalizes $M$ and $M^{\dagger}$ to $Z:=\operatorname{diag}\left(z_{1}, \ldots, z_{N}\right)$ and $\bar{Z}:=\operatorname{diag}\left(\overline{z_{1}}, \ldots, \overline{z_{N}}\right)$, respectively. ${ }^{1}$ Hence, we have

$$
M=U^{\dagger} Z U
$$

and

$$
M^{\dagger}=U \bar{Z} U^{\dagger}
$$

Note that $U$ in (7-5) and (7-6) is not unique, since there is freedom to multiply it on the left by a diagonal unitary matrix of $N$ arbitrary phases. Therefore, in order to count the number of degrees of freedom in $M$, we have to consider the $2 N$ real and imaginary parts of of the eigenvalues, plus the $N^{2}$ independent real parameters of $U$, subtracted by the $N$ arbitrary phases, adding up to $N(N+1)$ independent real degrees of freedom.

So the measure and the weight in (7-3) are invariant and consequently, one can simply derive the Jacobian for the transformation to the eigenvalues. The integration measure $\mathrm{d} M$ in (7-3) factorizes to

$$
\mathrm{d} M=\mathrm{d} U \prod_{1 \leq i<j \leq N}\left|z_{i}-z_{j}\right|^{2} \prod_{k=1}^{N} \mathrm{~d}^{2} z_{k} .
$$

The partition function (7-3) thus, up to a multiplicative constant factor, reads

$$
Z_{N}=\int_{\mathbb{C}} \prod_{k=1}^{N} \mathrm{~d} \mu\left(z_{k}\right)\left|\Delta\left(z_{1}, \ldots, z_{N}\right)\right|^{2},
$$

in which the Lebesgue measure $\mathrm{d} \mu(z)$ is given by

$$
\mathrm{d} \mu(z):=e^{-N V(z)} \mathrm{d}^{2} z .
$$

\section{2}

\section{Saddle Point Method}

Hence, once again, in the large $N$ limit, one can apply the saddle pint method by rewriting the partition function (7-3) as

$$
Z_{N}=\int_{\mathbb{C}} \mathrm{d}^{2} z_{k} e^{-N^{2} S_{e f f}\left(z_{1}, \ldots, z_{N}\right)}
$$

${ }^{1}$ Normal matrices are the largest class of matrices that can be diagonalized by unitary transformations $[57,80]$. 
where we have defined

$$
S_{e f f}\left(z_{1}, \ldots, z_{N}\right):=\frac{1}{N} \sum_{i=1}^{N} V\left(z_{i}\right)-\frac{2}{N^{2}} \sum_{1 \leq i<j \leq N} \ln \left|z_{i}-z_{j}\right|
$$

In this large $N$ limit, the eigenvalue density

$$
\rho_{N}(z):=\mathbb{E}\left[\frac{1}{N} \sum_{i=1}^{N} \delta^{(2)}\left(z-z_{i}\right)\right]
$$

becomes a continuous function $\rho(z)$ which satisfies the normalization condition

$$
\int_{\mathbb{C}} \mathrm{d}^{2} z \rho(z)=1
$$

Furthermore, in the limit $N \rightarrow \infty$, the effective action $S_{\text {eff }}\left(z_{1}, \ldots, z_{N}\right)$ turns into a functional $S_{\text {eff }}[\rho]$, given by

$$
S_{e f f}[\rho]=\int_{\mathbb{C}} \mathrm{d}^{2} z \rho(z) V(z)-\int_{\mathbb{C} \times \mathbb{C}} \mathrm{d}^{2} z \mathrm{~d}^{2} z^{\prime} \rho(z) \rho\left(z^{\prime}\right) \ln \left|z-z^{\prime}\right|,
$$

in which the potential is

$$
V(z)=2 \int_{\mathbb{C}} \mathrm{d}^{2} z^{\prime} \rho\left(z^{\prime}\right) \ln \left|z-z^{\prime}\right|
$$

Finding the saddle point configuration which minimizes the effective action functional (7-14), together with the constraint (7-13), is one of the central objectives in potential theory [81]. In fact, one has to solve the functional saddle point equation

$$
\begin{aligned}
\frac{\delta}{\delta \rho(z)}\left[\int_{\mathbb{C}} \mathrm{d}^{2} z^{\prime} \rho\left(z^{\prime}\right) V\left(z^{\prime}\right)-f_{\mathbb{C} \times \mathbb{C}} \mathrm{d}^{2} z^{\prime} \mathrm{d}^{2} z^{\prime \prime} \rho\left(z^{\prime}\right) \rho\left(z^{\prime \prime}\right) \ln \left|z^{\prime}-z^{\prime \prime}\right|\right. \\
\left.+c\left(\int_{\mathbb{C}} \mathrm{d}^{2} z^{\prime} \rho\left(z^{\prime}\right)-1\right)\right]=0
\end{aligned}
$$

where $c \in \mathbb{R}$ is a constant. Taking the functional derivative in (7-16), one has:

$$
\begin{aligned}
\int_{\mathbb{C}} \mathrm{d}^{2} z^{\prime}\left(\frac{\delta \rho\left(z^{\prime}\right)}{\delta \rho(z)} V\left(z^{\prime}\right)+\rho\left(z^{\prime}\right) \frac{\delta V\left(z^{\prime}\right)}{\delta \rho(z)}\right) \\
-\int_{\mathbb{C} \times \mathbb{C}} \mathrm{d}^{2} z^{\prime} \mathrm{d}^{2} z^{\prime \prime}\left(\frac{\delta \rho\left(z^{\prime}\right)}{\delta \rho(z)} \rho\left(z^{\prime \prime}\right)+\rho\left(z^{\prime}\right) \frac{\delta \rho\left(z^{\prime \prime}\right)}{\delta \rho(z)}\right) \ln \left|z^{\prime}-z^{\prime \prime}\right|+c \int_{\mathbb{C}} \mathrm{d}^{2} z^{\prime} \frac{\delta \rho\left(z^{\prime}\right)}{\delta \rho(z)}=0
\end{aligned}
$$

or 


$$
\begin{aligned}
\int_{\mathbb{C}} \mathrm{d}^{2} z^{\prime} \delta^{(2)}\left(z-z^{\prime}\right) V\left(z^{\prime}\right) & \\
-\int_{\mathbb{C} \times \mathbb{C}} \mathrm{d}^{2} z^{\prime} \mathrm{d}^{2} z^{\prime \prime}\left(\delta^{(2)}\left(z-z^{\prime}\right) \rho\left(z^{\prime \prime}\right)+\right. & \left.\rho\left(z^{\prime}\right) \delta^{(2)}\left(z-z^{\prime \prime}\right)\right) \ln \left|z^{\prime}-z^{\prime \prime}\right| \\
& +c \int_{\mathbb{C}} \mathrm{d}^{2} z^{\prime} \delta^{(2)}\left(z^{\prime}-z^{\prime \prime}\right)=0
\end{aligned}
$$

which yields

$$
V(z)-f_{\mathbb{C}} \mathrm{d}^{2} z^{\prime \prime} \rho\left(z^{\prime \prime}\right) \ln \left|z-z^{\prime \prime}\right|-f_{\mathbb{C}} \mathrm{d}^{2} z^{\prime} \rho\left(z^{\prime}\right) \ln \left|z-z^{\prime}\right|+c=0 .
$$

This, in turn, simplifies to

$$
V(z)=2 \int_{\mathbb{C}} \mathrm{d}^{2} z^{\prime} \rho\left(z^{\prime}\right) \ln \left|z-z^{\prime}\right|-c .
$$

Taking derivative of both sides of (7-20) with respect to $z$, we get

$$
\frac{\partial V(z)}{\partial z}=2 f_{\mathbb{C}} \mathrm{d}^{2} z^{\prime} \frac{\rho\left(z^{\prime}\right)}{z-z^{\prime}} .
$$

By applying $\partial / \partial z^{\prime}$ to both sides of (7-21), the above equation can be solved in terms of the density of eigenvalues (c.f. (6-6)):

$$
\rho(z)= \begin{cases}\frac{1}{\pi} \frac{\partial^{2} V(z)}{\partial z \partial \bar{z}}, & z \in D, \\ 0, & z \in \mathbb{C} \backslash D,\end{cases}
$$

in which the domain $D$ is determined by the condition [82]

$$
\oint_{\partial D} \mathrm{~d}^{2} z^{\prime} \frac{\partial V\left(z^{\prime}\right) / \partial z^{\prime}}{z-z^{\prime}}=0
$$

which can be derived from (7-21) with the help of the Cauchy integral formula. For a potential with radial symmetry $V=V(r)$, provided that the following constraints are satisfied: ${ }^{2}$

$$
\lim _{r \rightarrow \infty}\left(V(r)-\ln r^{2}\right)=\infty
$$

and

$$
r V^{\prime}(r) \text { increasing in } \mathbb{R}^{+} \text {, or } V^{\prime}(r)>0 \text { and } V \text { convex in } \mathbb{R}^{+}
$$

this equation gives (see the footnote at page 86)

$$
\rho(r)=\frac{1}{4 \pi r} \frac{\mathrm{d}}{\mathrm{d} r}\left(r V^{\prime}(r)\right) \mathbb{I}_{\left\{c_{-} \leq r \leq c_{+}\right\}} .
$$

${ }^{2}$ This result can be obtained by the theory of harmonic analysis [81]. The first condition guarantees that the eigenvalue density has a compact support. Second condition will be derived later in Ch. 9 (see (9-60) and (9-61)). 

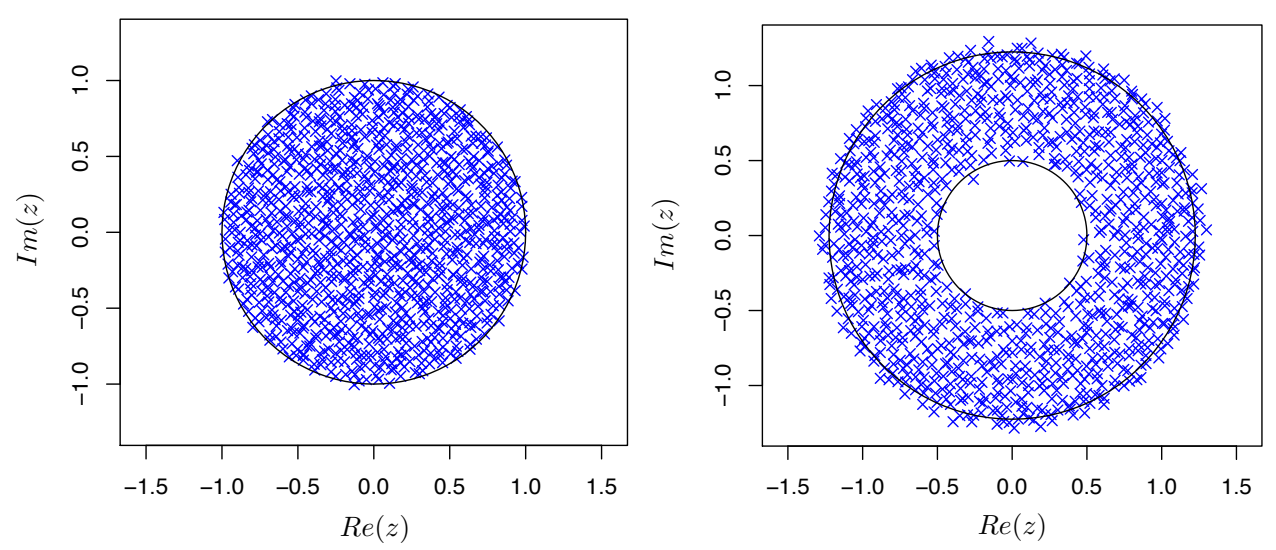

Figure 7.1: Left: Distribution of eigenvalues of a $1000 \times 1000$ Ginibre matrix equivalent to a Coulomb gas with Gaussian potential $V(r)=r^{2}$. The eigenvalues density is given by the circular law. Right: Distribution of eigenvalues of a $1000 \times 1000$ normal random matrix with potential $V(r)=r^{2}-r$ obtained using Monte-Carlo methods. The eigenvalue density has support on a ring.

Therefore, the eigenvalue density inherits the radial symmetry and is supported, in general, in a connected domain $D \subset \mathbb{C}$ [83]:

$$
D:=\left\{z \in \mathbb{C}: c_{-} \leq r \leq c_{+}\right\},
$$

in which the inner and outer radii of the support, $c_{-}$and $c_{+}$, satisfy the following equations [81, 83]:

$$
\begin{aligned}
V^{\prime}\left(c_{-}\right) & =0, \\
c_{+} V^{\prime}\left(c_{+}\right) & =2 .
\end{aligned}
$$

As an example, we consider the one-parameter family of potentials

$$
V(r ; s)=r^{2}+s r
$$

Then, the endpoints of the eigenvalue support are

$$
c_{-}(s)=\max \{-s / 2,0\}, \quad c_{+}(s)=\frac{1}{4}\left(\sqrt{s^{2}+16}-s\right) .
$$

The first of these equations, tells us that the domain over which the eigenvalues spread, undergoes a change of topology, depending on the value of $s$ : the eigenvalue density is supported on a disk for $s \geq 0$ (see Fig. 7.1 left), and on an annulus for $s<0$ (see Fig. 7.1 right). Furthermore, we notice that in the case of Gaussian potential, i.e., when $s=0$, one retrieves the circular law for the Ginibre ensemble:

$$
\rho(z)= \begin{cases}\frac{1}{\pi}, & |z| \leq 1 \\ 0, & |z|>1\end{cases}
$$


This means that the eigenvalues spread uniformly over the unit disk in the complex $z$ plane.

\section{3}

\section{Orthogonal Polynomials Method}

As previous section, our main objective is to obtain the eigenvalue density. First, we define and construct the integral kernel in terms of orthogonal polynomials. Next, we show how one can obtain the eigenvalue density from the integral kernel.

Based on the eigenvalue representation of the partition function, (7-8), we can employ the method of orthogonal polynomials to re-express the Vandermonde determinant in (7-8) in terms of them. In order to do this, we define an inner product $\langle\cdot \mid \cdot\rangle_{\mu}$ with respect to the measure $\mu$ as

$$
\langle f \mid g\rangle_{\mu}:=\int_{D} \mathrm{~d} \mu(z) f(z) \overline{g(z)}, \quad D \subset \mathbb{C} .
$$

Then, we denote the orthogonal polynomials associated with the measure (7-9) and with leading coefficients one by $P_{n}(z)$ which satisfy

$$
\left\langle P_{n} \mid P_{m}\right\rangle_{\mu}:=\int_{D} \mathrm{~d} \mu(z) P_{n}(z) \overline{P_{m}(z)}=\delta_{n m} h_{n}, \quad h_{n} \in \mathbb{R}^{+},
$$

where $h_{n}$ 's are the normalization constants. Obviously, Polynomials $\Pi(z)$ defined as

$$
\Pi_{n}(z):=\frac{P_{n}(z)}{\sqrt{h_{n}}}
$$

are orthonormal with respect to the measure (7-9), i.e.,

$$
\int_{D} \mathrm{~d} \mu(z) \Pi_{n}(z) \overline{\Pi_{m}(z)}=\delta_{n m}
$$

As an example, we consider Gaussian potential $V(z) \equiv V(|z|)=|z|^{2}$. In order to obtain the orthonormal polynomials in this case, first we notice that for any rotationally symmetric potential $V=V(r)$, where $r \equiv|z|$, and rotationally symmetric domain $D$, the monomials $z^{n}, n=0,1,2, \ldots$ are orthogonal. Therefore, we just need to calculate the normalization constants $h_{n}$. In the Gaussian case we are considering, we can find them as follows:

$$
\begin{aligned}
h_{n}=\left\langle z^{n} \mid z^{n}\right\rangle_{\mu}=\int_{\mathbb{C}} \mathrm{d} \mu(z) z^{n} \bar{z}^{n} & =\int_{\mathbb{C}} \mathrm{d}^{2} z e^{-N|z|^{2}}|z|^{2 n} \\
& =\int_{0}^{2 \pi} \mathrm{d} \theta \int_{0}^{\infty} \mathrm{d} r e^{-N r^{2}} r^{2 n+1} \\
& =2 \pi \int_{0}^{\infty} \mathrm{d} r e^{-N r^{2}} r^{2 n+1} \\
& =\pi n ! N^{-(n+1)} .
\end{aligned}
$$


Therefore, the orthonormal polynomials are

$$
\Pi_{n}(z)=\sqrt{\frac{N^{n+1}}{\pi n !}} z^{n}
$$

More generally, for a potential $V$ with radial symmetry, one can calculate the inner product $\left\langle z^{n} \mid z^{m}\right\rangle_{\mu}$, for $D=\mathbb{C}$ :

$$
\begin{aligned}
\left\langle z^{n} \mid z^{m}\right\rangle_{\mu} & =\int_{\mathbb{C}} \mathrm{d} \mu z^{n} \bar{z}^{m} \\
& =\int_{\mathbb{C}} \mathrm{d}^{2} z e^{-N V(z)} z^{n} \bar{z}^{m} \\
& =\int_{0}^{2 \pi} \mathrm{d} \theta e^{i \theta(n-m)} \int_{0}^{\infty} \mathrm{d} r r^{n+m+1} e^{-N V(r)} \\
& =2 \pi \delta_{n m} \int_{0}^{\infty} \mathrm{d} r r^{2 n+1} e^{-N V(r)}=\delta_{n m} h_{n}
\end{aligned}
$$

Consequently, the orthonormal polynomials $\Pi_{k}(z)$ are

$$
\Pi_{k}(z)=\frac{z^{k}}{\sqrt{h_{k}}}, \quad k=0,1,2, \ldots
$$

From the orthonormal polynomials $\Pi_{k}(z)$ for the potential $V$ and $D \subset \mathbb{C}$, we can construct the unnormalized reproducing integral kernel $\widetilde{K}_{N}$ :

$$
\widetilde{K}_{N}(z, w):=\sum_{k=0}^{N-1} \Pi_{k}(z) \overline{\Pi_{k}(w)}, \quad z, w \in D,
$$

which satisfies the following identity [84]

$$
\int_{D} \mathrm{~d}^{2} z e^{-N V(z)} \tilde{K}_{N}(z, z)=N
$$

One can also define the normalized reproducing kernel $K_{N}$ :

$$
\begin{aligned}
K_{N}(z, w) & :=e^{-\frac{N}{2}(V(z)+\overline{V(w)})} \tilde{K}_{N}(z, w) \\
& =e^{-\frac{N}{2}(V(z)+\overline{V(w)})} \sum_{k=0}^{N-1} \Pi_{k}(z) \overline{\Pi_{k}(w)}, \quad z, w \in D
\end{aligned}
$$

From the above definition, one immediately concludes ${ }^{3}$

$$
K_{N}(z, w)=\overline{K_{N}(w, z)}
$$

Besides, one has

${ }^{3}$ When the potential $V$ is real, i.e., $V(z)=\overline{V(z)}, \forall z \in D$, this identity becomes $K_{N}(z, w)=\overline{K_{N}(z, w)}$. 


$$
\begin{aligned}
\int_{D} \mathrm{~d}^{2} z K_{N}(z, z) & =\int_{D} \mathrm{~d}^{2} z e^{-N V(z)} \sum_{k=0}^{N-1}\left|\Pi_{k}(z)\right|^{2} \\
& =\sum_{k=0}^{N-1} \int_{D} \mathrm{~d}^{2} z e^{-N V(z)}\left|\Pi_{k}(z)\right|^{2} \\
& =\sum_{k=0}^{N-1} \delta_{k k}=N
\end{aligned}
$$

where in the last line we have used the orthonormality of the polynomials $\Pi_{k}(z)$ with respect to the measure $e^{-N V(z)} \mathrm{d}^{2} z$. Furthermore, from the orthonormality of $\Pi_{k}(z)$ 's, we can derive the reproducing property of the integral kernel $K_{N}(z, w)$ :

$$
\begin{aligned}
& \int_{D} \mathrm{~d}^{2} w \overline{K_{N}(w, z)} K_{N}(w, u)=e^{-\frac{N}{2}(V(z)+\overline{V(u)})} \sum_{k, l=0}^{N-1} \Pi_{l}(z) \overline{\Pi_{k}(z)} \times \\
& \underbrace{\int_{D} \mathrm{~d}^{2} w e^{-N V(w)} \overline{\Pi_{l}(w)} \Pi_{k}(w)}_{\delta_{k l}} \\
&=e^{-\frac{N}{2}(V(z)+\overline{V(u)})} \sum_{k=0}^{N-1} \Pi_{k}(z) \overline{\Pi_{k}(u)}=K_{N}(z, u) .
\end{aligned}
$$

As an example, for the Gaussian potential $V(z)=|z|^{2}$, one has:

$$
\begin{aligned}
K_{N}(z, w) & =e^{-\frac{N}{2}\left(|z|^{2}+|w|^{2}\right)} \sum_{k=0}^{N-1}\left(\sqrt{\frac{N^{k+1}}{\pi k !}} z^{k} \sqrt{\frac{N^{k+1}}{\pi k !}} \bar{w}^{k}\right) \\
& =\frac{N}{\pi} e^{-\frac{N}{2}\left(|z|^{2}+|w|^{2}\right)} \sum_{k=0}^{N-1} \frac{(N z \bar{w})^{k}}{k !}
\end{aligned}
$$

More generally, for an arbitrary potential with radial symmetry, one has

$$
\begin{aligned}
K_{N}(z, w) & =e^{-\frac{N}{2}(V(|z|)+\overline{V(|w|)})} \sum_{k=0}^{N-1} \Pi_{k}(z) \overline{\Pi_{k}(w)} \\
& =e^{-\frac{N}{2}(V(|z|)+\overline{V(|w|)})} \sum_{k=0}^{N-1} \frac{(z \bar{w})^{k}}{2 \pi I_{k}}
\end{aligned}
$$

where $I_{k}$ is defined in (7-39).

Knowing the integral kernel, one can obtain the eigenvalue density. In fact, ne can express the density of eigenvalues in terms of the integral kernel as

$$
\rho_{N}(z)=\frac{K_{N}(z, z)}{N} .
$$


Then, it immediately follows from (7-45) that the eigenvalue density is normalized: ○

$$
\int_{D} \mathrm{~d}^{2} z \rho(z)=1
$$

Therefore, in the limit $N \rightarrow \infty$, the eigenvalue density is obtained as

$$
\rho(z)=\lim _{N \rightarrow \infty} \rho_{N}(z)=\lim _{N \rightarrow \infty} \frac{K_{N}(z, z)}{N} .
$$

Let's consider the Gaussian potential $V(z)=|z|^{2}$ as an example. From (7-47) we get

$$
\rho_{N}(z)=\frac{1}{\pi} e^{-N|z|^{2}} \sum_{k=0}^{N-1} \frac{N^{k}|z|^{2 k}}{k !}
$$

and in the limit $N \rightarrow \infty$,

$$
\rho(z)=\frac{1}{\pi} \lim _{N \rightarrow \infty} \frac{N^{N+1}}{N !} \int_{|z|^{2}}^{\infty} \mathrm{d} \xi \xi^{N-1} e^{-N \xi}
$$

Now, we can use the Stirling's approximation for the factorial:

$$
N ! \sim \sqrt{2 \pi} N^{N+1 / 2} e^{-N}, \quad N \rightarrow \infty .
$$

Substituting (7-54) in (7-53), we get

$$
\rho(z)=\frac{1}{\pi} \lim _{N \rightarrow \infty} \sqrt{N / 2 \pi} \int_{|z|^{2}}^{\infty} \mathrm{d} \xi \frac{e^{-N(\xi-1-\ln \xi)}}{\xi} .
$$

Since $N \rightarrow \infty$, we can apply the saddle point method. Then, changing the integration variable as $\xi-1=: \eta$ yields

$$
\rho(z)=\frac{1}{\pi} \lim _{N \rightarrow \infty} \sqrt{N / 2 \pi} \int_{|z|^{2}-1}^{\infty} \mathrm{d} \eta \frac{e^{-N \eta^{2} / 2}}{\eta+1} .
$$

This last integral can be obtained by contour integration in the complex $\eta$ plane. The result is [84]

$$
\rho(\lambda)= \begin{cases}\frac{1}{\pi}, & |z|<1 \\ \frac{1}{2 \pi}, & |z|=1 \\ 0, & |z|>1\end{cases}
$$




\section{8 \\ Extreme Value Statistics of GUE}

In this chapter, we first review the Tracy-Widom law for the EVS of three classical Gaussian ensembles. Then we follow the approach of [14] by which non-Gaussian potential can be considered too, via Lax method. This method lets us avoid the complicated formalism of Fredholm determinant of Airy kernel.

\section{1}

\section{Tracy-Widom Law for the Classical Gaussian Ensembles}

We recall that the eigenvalue representation of the partition function of GUE with potential $V(M)=M^{2}$ is $($ see $(5-9))$

$$
Z_{N}=\frac{1}{N !} \int_{-\infty}^{\infty} \prod_{i=1}^{N} \mathrm{~d} \lambda_{i} e^{-N \lambda_{i}^{2}} \prod_{1 \leq j<k \leq N}\left(\lambda_{k}-\lambda_{j}\right)^{2} .
$$

We would like to study the statistics of the extreme eigenvalue

$$
\lambda_{\max }:=\max \left\{\lambda_{1}, \ldots, \lambda_{N}\right\} .
$$

In the absence of the Vandermonde determinant in (8-1), the partition function would factorize and the eigenvalues would be independent Gaussian random variables. As we already studied in Ch. 3, for such independent and identically distributed random variables $\left\{\lambda_{i}\right\}_{i=1}^{N}$, the extreme value statistics is fully understood and depending on the tail of the distribution of individual $\lambda_{i}$, the distribution of the maximum eigenvalue, when properly rescaled, belongs to one of the three families of Gumbel, Fréchet and Weibull.

However, due to the presence of the Vandermonde determinant in (8-1), the eigenvalues are strongly correlated and there is no general theory for the distribution of the maximum of strongly correlated variables. However, in the case of Gaussian random matrices, the distribution of $\lambda_{\max }$ was derived in the seminal work by Tracy and Widom [85, 86] (see also [87] for a short review). The original derivation of the Tracy-Widom law for the scaled distribution of the largest eigenvalue of $\lambda_{\max }$ of a large complex Hermitian random matrix with Gaussian entries was based on nontrivial asymptotic analysis of the Fredholm determinant of the Airy Kernel. 


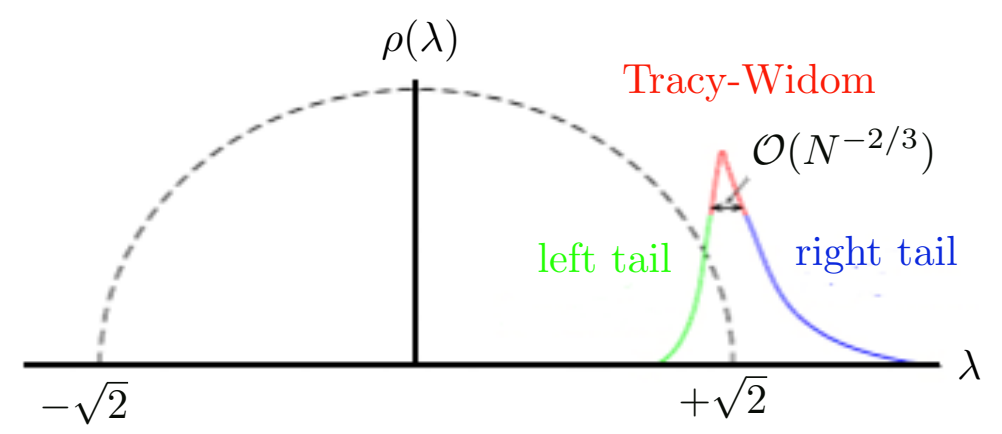

Figure 8.1: The average eigenvalue density of a Gaussian random matrix ensemble $\rho(\lambda)$ with a compact support $[-\sqrt{2}, \sqrt{2}]$. The eigenvalue with the largest modulus has an expectation value $\mathbb{E}\left[\lambda_{\max }\right]=\sqrt{2}$ and its distribution close to this expectation value has, over a scale $\mathcal{O}\left(N^{-2 / 3}\right)$, obeys TracyWidom law (red solid line). However, over a scale $\mathcal{O}(1)$, the distribution has large deviation tails shown by solid green (left large deviations) and solid blue (right large deviations) lines.

Several alternative derivations of the Tracy-Widom distribution exist which avoid Fredholm determinant formalism; e.g., orthogonal polynomials method [60] and loop equations approach $[88,89]$. In the former approach, the probability that there is no eigenvalue in the interval $(y, \infty)$ is calculated directly in terms of the norms of orthogonal polynomials on the complement interval $(-\infty, y]$ for a Gaussian potential.

Here, we follow the approach of [14] by which non-Gaussian potential can be considered too, via Lax method (see also [90]). But before, let us recall some known properties of the random variable $\lambda_{\max }$. Its average can be easily obtained from the outer edge of the eigenvalue support. For a $N \times N$ Hermitian random matrix with Gaussian potential $V(M)=M^{2}$, the eigenvalue density is given by the Wigner semi-circle law, in the large $N$ limit:

$$
\rho(\lambda)=\frac{1}{\pi} \sqrt{2-\lambda^{2}}
$$

which is supported on $[-\sqrt{2}, \sqrt{2}]$. It follows then, that the average value of the maximal eigenvalue $\lambda_{\max }$ is given, in the limit $N \rightarrow \infty$, by the outer edge of the eigenvalue support:

$$
\lim _{N \rightarrow \infty} \mathbb{E}\left[\lambda_{\max }\right]=\sqrt{2}
$$

However, $\lambda_{\max }$ fluctuates around this average value, and has a distribution around its mean value $\sqrt{2}$. The question is what the probability distribution of $\lambda_{\max }$ is, or in other words, the question is how $\mathbb{P}_{N}\left(\lambda_{\max } \leq y\right)$ behaves for large $N$. It turns out that the fluctuations of $\lambda_{\max }$ around its mean, have two scales for large $N[60,91,92]$ : 


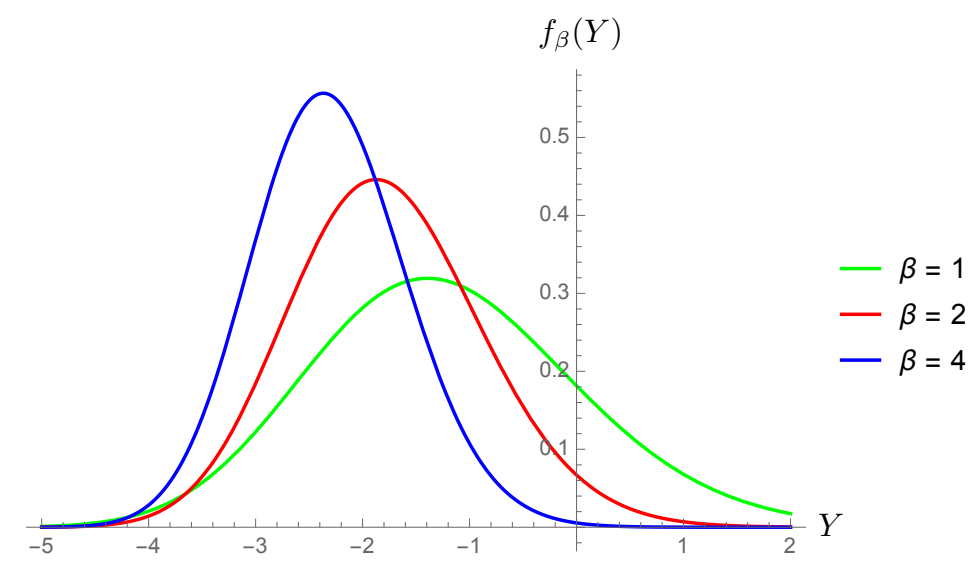

Figure 8.2: Largest eigenvalue densities $f_{\beta}(Y) \equiv \mathrm{d} F_{\beta}(Y) / \mathrm{d} Y$ for $\beta=1,2,4$.

typical small fluctuations, where $\lambda_{\max }=\mathcal{O}\left(N^{-2 / 3}\right)$, and atypical large fluctuations, where $\lambda_{\max }=\mathcal{O}(1)$ (see Fig. 8.1). Typical fluctuations of the maximum eigenvalue $\lambda_{\max } \leq y$ for the three Gaussian ensembles can be written as

$$
y=\sqrt{2}+a_{\beta} N^{-2 / 3} Y, \quad \beta=1,2,4,
$$

where $Y$ is the new scaled random variable which characterizes the typical fluctuations of the maximum eigenvalue. $a_{1,2}=1 / \sqrt{2}$ for GOE and GUE, and $a_{4}=2^{-7 / 6}$ for GSE. In $[85,86]$ it was shown that in the large $N$ limit, the distribution of $Y$ is independent of $N$, and depends explicitly on $\beta$ :

$$
\lim _{N \rightarrow \infty} \mathbb{P}_{N}\left(\lambda_{\max } \leq \sqrt{2}+a_{\beta} N^{-2 / 3} Y\right)=: F_{\beta}(Y) .
$$

$F_{\beta}(Y)$ is the cdf of the scaled random variable $Y$ and is called the Tracy-Widom distribution. For $\beta=2$, it is given by

$$
F_{2}(Y)=\exp \left(-\int_{Y}^{\infty} \mathrm{d} z(z-Y) q^{2}(z)\right)
$$

where $q(z)$ is the Hasting-MacLeod solution to the Painlevé II equation

$$
q^{\prime \prime}(z)=2 q^{3}(z)+z q(z)+\alpha
$$

with $\alpha=0$, which is uniquely determined by specifying the boundary condition $q(z) \sim \operatorname{Ai}(z)$, where $\operatorname{Ai}(z)$ is the Airy function satisfying the differential equation

$$
\operatorname{Ai}^{\prime \prime}(z)-z \operatorname{Ai}(z)=0
$$

with asymptotic behaviour

$$
\operatorname{Ai}(z) \sim \frac{1}{2 \sqrt{\pi} z^{1 / 4}} e^{-\frac{2}{3} z^{3 / 2}}, \quad(z \rightarrow \infty) .
$$

Furthermore, for $\beta=2,4$ one has

$$
F_{1}(Y)=F_{2}^{1 / 2}(Y) \exp \left(\frac{1}{2} \int_{Y}^{\infty} \mathrm{d} z q(z)\right)
$$


and

$$
F_{4}(Y)=F_{2}^{1 / 2}(Y) \cosh \left(\frac{1}{2} \int_{Y}^{\infty} \mathrm{d} z q(z)\right) .
$$

Corresponding largest eigenvalue densities are depicted in Fig. 8.2.

\section{2}

\section{Orthogonal Polynomials Approach to the EVS of GUE}

We are interested in the statistics of the largest eigenvalue $\lambda_{\max }$. The basic idea underlying this approach is simple. Let us define a partition function $Z_{N}(y)$ as

$$
Z_{N}(y):=\frac{1}{N !} \int_{-\infty}^{y} \prod_{i=1}^{N} \mathrm{~d} \lambda_{i} e^{-N V\left(\lambda_{i}\right)} \prod_{1 \leq j<k \leq N}\left(\lambda_{k}-\lambda_{j}\right)^{2} .
$$

This cut-off partition function $Z_{N}(y)$ is, in fact, the cdf of $\lambda_{\max }$ and can be interpreted as a partition function of a Coulomb gas in the presence of a hard wall at $y$. Then, $Z_{N}(\infty) \equiv Z_{N}$, i.e., when integrating over the entire real line, we recover the conventional partition function. It follows that cdf of the eigenvalue with the largest modulus is given by

$$
F_{N}(y):=\mathbb{P}_{N}\left(\lambda_{\max } \leq y\right)=\mathbb{P}\left(\lambda_{1} \leq y, \ldots, \lambda_{N} \leq y\right)=\frac{Z_{N}(y)}{Z_{N}(\infty)} .
$$

One possible way to determine the probability in (8-14) is to study the asymptotic behaviour of the orthogonal polynomials for the partition function $Z_{N}(\infty)$ in a proper scaling limit. However, we follow the approach of $[14,60]$ by introducing orthogonal polynomials for the cut-off partition function $Z_{N}(y)$.

We introduce a set of monic polynomials $\left\{P_{n}(\lambda)\right\}_{n=1}^{N}$ of degree $n$ that are orthogonal with respect to the measure $\mathrm{d} \mu(\lambda):=e^{-N V(\lambda)} \mathrm{d} \lambda$ :

$$
\left\langle P_{n} \mid P_{m}\right\rangle:=\int_{-\infty}^{y} \mathrm{~d} \lambda e^{-N V(\lambda)} P_{n}(\lambda) P_{m}(\lambda)=\delta_{n m} h_{n}
$$

in which $h_{n} \equiv h_{n}(y)>0$. Hence, $P_{n}=P_{n}(\lambda ; y)$ depends on $y$. For example, in the Gaussian case, when $V(\lambda)=\lambda^{2}$, the first two polynomials are given by [60]

$$
\begin{aligned}
& P_{0}(\lambda)=1 \\
& P_{1}(\lambda)=\lambda+\frac{e^{-N y^{2}}}{\sqrt{\pi N}(1+\operatorname{erf}(\sqrt{N} y))}
\end{aligned}
$$

Thus we get, for instance [60], 


$$
\begin{aligned}
& h_{0}=\frac{\sqrt{\pi}}{2 \sqrt{N}}(1+\operatorname{erf}(\sqrt{N} y)), \\
& h_{1}=\frac{-2 \sqrt{N} y e^{-N y^{2}}+\sqrt{\pi}(1+\operatorname{erf}(\sqrt{N} y))-\frac{2 e^{-2 N y^{2}}}{\sqrt{\pi}(1+\operatorname{erf}(\sqrt{N} y))}}{4} .
\end{aligned}
$$

In the limit $y \rightarrow \infty$, the Hermite polynomials are recovered (see Ch. 5). Next, we introduce the set of orthonormal polynomials $\Pi_{n}(\lambda)$ with the leading coefficient $1 / \sqrt{h_{n}}$

which satisfy

$$
\Pi_{n}(\lambda)=\frac{1}{\sqrt{h_{n}}} \lambda^{n}+\mathcal{O}\left(\lambda^{n-1}\right)
$$

$$
\left\langle\Pi_{n} \mid \Pi_{m}\right\rangle:=\int_{-\infty}^{y} \mathrm{~d} \lambda e^{-N V(\lambda)} \Pi_{n}(\lambda) \Pi_{m}(\lambda)=\delta_{n m} .
$$

Using orthonormality of $\Pi_{n}$ 's and following the analogous approach in Ch. 5, one can show that

$$
Z_{N}(y)=\prod_{k=0}^{N-1} h_{k}(y)=h_{0}^{N} \prod_{k=1}^{N-1}\left(r_{k}(y)\right)^{N-k}
$$

where we have defined the ratios

$$
r_{n}:=\frac{h_{n}}{h_{n-1}}, \quad n=1,2, \ldots,
$$

that will help us to determine the distribution of the largest eigenvalue.

In order to determine $r_{k}$ 's in a general setting that includes the case of the cut-off integral in (8-13), we also introduce differentiation with respect to the cut-off $y$, denoted by $C$ as

$$
C_{n m} \Pi_{m}(\lambda):=\partial_{y} \Pi_{n}(\lambda)
$$

or equivalently

$$
C_{n m}=\left\langle\Pi_{m} \mid \partial_{y} \Pi_{n}\right\rangle \text {. }
$$

Since the coefficient of the leading order term in (8-19) is dependent on $y$, the polynomial on the right hand side of (8-23) is at most of degree $n$, which means he matrix representing $C$ is lower triangular including the diagonal:

$$
C_{n, n+k}=0, \quad k>0
$$

with diagonal elements

$$
C_{n n}=\int_{-\infty}^{y} \mathrm{~d} \lambda e^{-N V(\lambda)} \Pi_{n}(\lambda) \partial_{y}\left(\frac{1}{\sqrt{h_{n}}}+O\left(\lambda^{n-1}\right)\right)=-\frac{1}{2} \partial_{y}\left(\ln h_{n}\right) .
$$


The commutation relation satisfied by $B$ (already introduced in Ch. 5) and $C$ can be derived easily:

$$
\begin{aligned}
(B C-C B)_{n m} \Pi_{m} & =B_{n l} \partial_{y} \Pi_{l}-\partial_{y}\left(\lambda \Pi_{n}\right) \\
& =B_{n l} \partial_{y} \Pi_{l}-\partial_{y}\left(B_{n l} \Pi_{l}\right) \\
& =-\Pi_{l} \partial_{y} B_{n l}=-\Pi_{m} \partial_{y} B_{n m}
\end{aligned}
$$

where in the second line we have used the definition of the operator $B$ and in the third line we have used the fact that the summation index $l$ is a dummy index and can be replaced by $m$. Equation (8-27) can be written in operator form as

$$
[B, C]=-\partial_{y} B
$$

\section{3}

\section{The String Equation in the Presence of a cut-off}

One can introduce an operator $H$ which leads to an algebraic equation in the coefficients of the recursion relation (5-78), $r_{n}$ and $s_{n}$, in the presence of a cutoff at $y[14]$ :

$$
\begin{aligned}
H_{n m} & :=(A(B-y))_{n m}-\frac{N}{2}\left(V^{\prime}(B)(B-y)\right)_{n m}+\frac{1}{2} \delta_{n m} \\
& =\int_{-\infty}^{y} \mathrm{~d} \lambda e^{-N V(\lambda)} \Pi_{m}(\lambda)\left((\lambda-y)\left(\partial_{\lambda}-\frac{N}{2} V^{\prime}(\lambda)\right)+\frac{1}{2}\right) \Pi_{n}(\lambda) .
\end{aligned}
$$

One can check that $H$ is antisymmetric, i.e.,

$$
H=-H^{\top},
$$

by expanding the derivative in the following identity:

$$
\int_{-\infty}^{y} \mathrm{~d} \lambda \partial_{\lambda}\left((\lambda-y) e^{-N V(\lambda)} \Pi_{n}(\lambda) \Pi_{m}(\lambda)\right)=0
$$

which gives

$$
H_{n m}+H_{m n}=0 .
$$

Since $A$ is strictly lower triangular and $B$ is tridiagonal, their product appearing in the first term on the right hand side of (8-29) has no contribution in the + part of $H$. Therefore, $H_{n, n+k}=-\frac{N}{2}\left(V^{\prime}(B)(B-y)\right)_{n, n+k}$ for all $k>0$. This result together with the antisymmetry of $H$ let us to write

$$
H=-\frac{N}{2}\left(V^{\prime}(B)(B-y)_{+}-V^{\prime}(B)(B-y)_{-}\right),
$$


which implies that $H$ has a finite number of non-zero off-diagonal elements. For example, in the Gaussian case, it reads

$$
\begin{aligned}
H_{n m}^{\text {Gausss }}= & -N \alpha\left(\sqrt{r_{n+1} r_{n+2}} \delta_{n+2, m}+\sqrt{r_{n+1}}\left(s_{n+1}+s_{n}-y\right) \delta_{n+1, m}\right) \\
& +N \alpha\left(\sqrt{r_{n}}\left(s_{n}+s_{n-1}-y\right) \delta_{n-1, m}+\sqrt{r_{n-1} r_{n}} \delta_{n-2, m}\right) .
\end{aligned}
$$

Furthermore, upon the definition of $H$ in (8-29), and the commutation relations (5-86) and (8-28), we can derive the following commutation relation: ${ }^{1}$

$$
[B-y, H]=B-y,
$$

which will be referred to as string equation, and provides algebraic relations between the recurrence coefficients, i.e., it do not contain derivatives. We can again consider the Gaussian potential as an example. Inserting (5-80) and (8-34) into the string equation (8-35), we obtain five equations, two of which are identically satisfied for $m=n \pm 2$. The equations for $m=n \pm 1$ are the same and we are left with two equations for $m=n$ and $m=n+1$ :

$$
s_{n+1}^{2}-s_{n}^{2}+r_{n+2}-r_{n}-y\left(s_{n+1}-s_{n}\right)=\frac{1}{N},
$$

and

$$
r_{n+1}\left(s_{n+1}+s_{n}-y\right)-r_{n}\left(s_{n}+s_{n+1}-y\right)=\frac{1}{2 N}\left(s_{n}-y\right) .
$$

\section{4}

\section{Double Scaling Limit of the String Equation}

Now, we are going to study the large $N$ limit behaviour of (8-14), in which the partition function is given by (8-21). Hence, we have to determine the asymptotics of the recurrence coefficients in (5-80). As we recall, in the case of Gaussian potential and in the absence of a hard wall, i.e., when $y \rightarrow \infty$, the eigenvalue density $\rho(\lambda)$ is given by the Wigner semi-circle law that vanishes as a square root at the edges of its support. By taking the limit $N \rightarrow \infty$ and simultaneously letting $y$ approach the outer edge of the support - and hence, the name double scaling limit - the distribution of the largest eigenvalue can be found.

For the Gaussian potential, a non-trivial limit can be taken if we make the following scaling ansatz [93-95]:

\footnotetext{
${ }^{1}$ We have added a trivial zero $0=[-y, H]$.
} 


$$
\begin{aligned}
\xi & =1-\frac{1}{N} X, \\
y & =y_{c}+\frac{c}{N} Y,
\end{aligned}
$$

where $\xi:=n / N \in[0,1]$ is a continuous variable in the limit $N \rightarrow \infty$. Furthermore, $y_{c} \equiv \mathbb{E}\left[y_{\max }\right]=c_{+}$, and $c$ is an arbitrary constant which will be determined later. In addition, we make the following ansatz for the recurrence coefficients $r_{n}$ and $s_{n}$, which now become functions of the continuous variables $\xi$ an $y$ :

$$
\begin{aligned}
r(\xi, y) & =r_{c}\left(1+\frac{1}{N} \rho(X, Y)+\mathcal{O}\left(N^{-3 / 2}\right)\right) \\
s(\xi, y) & =\sqrt{r_{c}}\left(s_{c}+\frac{1}{N} \sigma(X, Y)+\mathcal{O}\left(N^{-3 / 2}\right)\right) .
\end{aligned}
$$

it will also be useful to define new functions $u$ and $v$ as

$$
u:=\rho+\sigma, \quad v:=\rho-\sigma .
$$

Following [60], we can insert (8-38) and (8-39) into the string equation (8-35), and then Taylor expand to derive the differential equations for the scaling functions $u$ and $v$. However, following [90], the string equation (8-35) can be solved using pseudo-differential equations $[96,97]$. For example, in the case $y=\infty$, one can solve the string equation either by substituting the above ansatz in it, or using pseudodifferential operators. The latter approach yields the following equations for the Gaussian potential [90]:

$$
\begin{aligned}
& \mathcal{L}_{1}[u(X, \infty)]=X / 2, \\
& \mathcal{L}_{1}[v(X, \infty)]=0
\end{aligned}
$$

in which $\mathcal{L}_{k}$ is the Lenard differential operator defined in (8-50).

In the large $N$ double scaling limit, the matrices $B$ and $H$ which all have only a finite number non-zero off diagonals, scale to differential operators of finite order. The commutation relations derived in the previous section, then allow us to directly obtain a differential equation for the scaling function. First, we consider the scaling limit of the matrix $B$. Using general scaling ansatz (8-38), it scales to $[90,96]$

$$
B \rightarrow\left(B_{c}+\frac{1}{N} \sqrt{r_{c}} \mathcal{B}+\mathcal{O}\left(N^{-2}\right)\right)
$$

where we have defined the operators $B_{c}:=\sqrt{r_{c}}\left(2+s_{c}\right)$ and $\mathcal{B}:=\mathrm{d}^{2}+u(X)$, in which $\mathrm{d} \equiv \mathrm{d} / \mathrm{d} X$. For Gaussian potential, the operator $H$ also scales to a differential 
operator of a fixed degree $m$ as follows [90]:

$H \rightarrow N^{-(m-3) / 2} c c^{\prime}\left(\mathrm{d}^{m}+\cdots\right)+\mathcal{O}\left(N^{-1-(m-3) / 2}\right)=: N^{-(m-3) / 2} \mathcal{H}+\mathcal{O}\left(N^{-1-(m-3) / 2}\right)$

where $c^{\prime}$ is a constant. Hence, we can write (8-35) as

$$
\frac{1}{N}\left(N^{-(m-3) / 2}[\mathcal{B}, \mathcal{H}]+\mathcal{O}\left(N^{-1-(m-3) / 2}\right)\right)=\frac{1}{N}\left(\mathrm{~d}^{2}+u-Y\right)+\mathcal{O}\left(N^{-2}\right),
$$

in which $c$ is fixed as $c=\sqrt{r_{c}}$ for convenience. Furthermore, defining $\overline{\mathcal{H}}$ as

$$
\mathcal{H}=: \overline{\mathcal{H}}+\frac{1}{4}\{X, d\}
$$

in which $\{$,$\} denotes the anti-commutator, one can show that$

$$
[\mathcal{B}, \overline{\mathcal{H}}]=\frac{1}{2} X u^{\prime}(X)+u(X)-Y
$$

In the case of Gaussian potential, the most general form of $\overline{\mathcal{H}}$ for which the above equation is satisfied is [90]

$$
\overline{\mathcal{H}}=c c^{\prime}\left(\left(\mathcal{B}^{3 / 2}\right)_{+}-Y\left(\mathcal{B}^{3 / 2}\right)_{-}\right)
$$

Furthermore, one can use the fact that $[90,97]$

$$
\left[\mathcal{B},\left(\mathcal{B}^{(2 k-1) / 2}\right)_{+}\right]=-4^{1-k} \mathcal{L}_{k}^{\prime}[u], \quad(' \equiv \mathrm{d}),
$$

where $\mathcal{L}_{k}$ is Lenard differential operator, defined by the following recursion relation

$$
\mathcal{L}_{k+1}^{\prime}[f] \equiv \mathrm{d} \mathcal{L}_{k+1}[f]=\left(\mathrm{d}^{3}+4 f \mathrm{~d}+2 f^{\prime}\right) \mathcal{L}[f], \quad\left(\mathcal{L}_{0}=1 / 2\right)
$$

with the condition $\mathcal{L}_{k}[0]=0$ for all $k$. Now, under a proper rescaling of $X$ and $u$, the equation (8-47) becomes [90]:

$$
\mathcal{L}_{2}^{\prime}[u]-4 Y \mathcal{L}_{1}^{\prime}[u]=X u^{\prime}(X)+2 u(X)-2 Y,
$$

which is the differential equation that the scaling function $u$ has to satisfy for the Gaussian potential.

Now, we write the equation (8-21) as

$$
\ln \left(Z_{N}(y)\right)=N \ln h_{0}+N \sum_{i=1}^{N-1}\left(1-\frac{1}{N}\right) \ln r_{i}
$$

In order to consider the double scaling limit of the above formula, we take the limit $N \rightarrow \infty$ at first, which using Euler-McLaurin formula reads (see App. A):

$$
\ln \left(Z_{N}(y)\right)=N^{2} \int_{0}^{1} \mathrm{~d} \xi(1-\xi) \ln (r(\xi, y))+\mathcal{O}\left(N^{-1}\right),
$$

which after substituting the scaling ansatz takes the form 


$$
\ln (Z(Y))=-\int_{-\infty}^{0} \mathrm{~d} X \frac{X}{2}(u(X, Y)-v(X, Y)) .
$$

On the other hand, one can show that $v$ still satisfies (8-42) for finite $y$. therefore, by imposing the same boundary conditions on $v$ as the case of $y \rightarrow \infty$, we obtain for the double scaling limit of probability distribution of the eigenvalue with largest modulus

$$
\ln (\mathbb{P}(Y))=-\int_{-\infty}^{0} \mathrm{~d} X \frac{X}{2}(u(X, Y)-u(X, \infty)) .
$$

Equation (8-55) together with the equation (8-51) provide an expression for the probability distribution of the eigenvalue with largest modulus. In fact, for the pdf of the largest eigenvalue we have

$$
\frac{\mathrm{d}}{\mathrm{d} Y} \ln (\mathbb{P}(Y))=-\int_{-\infty}^{0} \mathrm{~d} X \frac{X}{2} \partial_{Y} u(X, Y),
$$

which using $\partial_{Y} u(X, Y)=\partial_{X}\left(X-2 \mathcal{L}_{1}[u]\right)$ [90] becomes ${ }^{2}$

$$
\frac{\mathrm{d}}{\mathrm{d} Y} \ln (\mathbb{P}(Y))=-\int_{-\infty}^{0} \mathrm{~d} X\left(\mathcal{L}_{1}[u]-\frac{1}{2}\right) .
$$

We recall that $u$ satisfies the equation (8-41), hence,

$$
U(X, \infty)=\frac{X}{2}
$$

$u(X, Y)$ also satisfies (8-51), which reads

$$
u^{(3)}+6 u u^{\prime}-(X+4 Y) u^{\prime}-2 u+2 Y=0 .
$$

If we perform the following rescaling

$$
u(X, Y)=: \gamma^{2} \bar{u}\left(\gamma X, \gamma^{-2} Y\right), \quad \gamma=-2^{-1 / 3},
$$

then (8-59) becomes

$$
\bar{u}^{(3)}+2 \bar{u}\left(2+3 \bar{u}^{\prime}\right)+2(X-2 Y) \bar{u}^{\prime}-4 Y=0 .
$$

Now if we let $-2 q^{2}(X, Y):=\bar{u}(X, Y)+X$, and substitutue it into the above equation, we find that $q(X, Y)=q(X+Y, 0) \equiv q(X+Y)$ satisfies

$$
q(X) W^{\prime}(X)=-3 q^{\prime}(X) W(X)
$$

where $W(X) \equiv q^{\prime \prime}(X)-2 q^{3}(X)-X q(X)$. Integrating this equation shows that $q(X)$ satisfies the Painlevé II equation with $\alpha=0$. Now we can write (8-55) as

$$
\begin{aligned}
\ln (\mathbb{P}(Y)) & =-\int_{-\infty}^{0} \mathrm{~d} X \frac{X}{2}\left(u(X, Y)-\frac{X}{2}\right) \\
& =-\int_{-\infty}^{0} \mathrm{~d} X \frac{X}{2}\left(\gamma^{2} \bar{u}\left(\gamma X, \gamma^{-2} Y\right)-\frac{X}{2}\right) .
\end{aligned}
$$

${ }^{2}$ The boundary term vanishes due to the boundary conditions on $u$. 
After changing the integration variable as $\bar{X}:=\gamma X$, we have

$$
\begin{aligned}
\ln (\mathbb{P}(Y)) & =\int_{0}^{\infty} \mathrm{d} \bar{X} \frac{\bar{X}}{2}\left(\bar{u}\left(\bar{X}, \gamma^{-2} Y\right)+\bar{X}\right) \\
& =-\int_{0}^{\infty} \mathrm{d} X X q^{2}\left(X+\gamma^{-2} Y\right)
\end{aligned}
$$

One therefore has

$$
\ln (\mathbb{P}(Y))=\int_{Y}^{\infty} \mathrm{d} X(Y-X) q^{2}(X),
$$

which is the celebrated Tracy-Widom distribution. 


\section{Extreme Value Statistics of Random Normal Matrices}

We introduced and studied random normal matrices in Ch. 7. The extreme value statistics of GUE was also discussed in the previous chapter. The next natural step is to study the extreme value statistics of random normal matrices.

In this chapter, after a brief review to normal normal matrices and their relation to $2 D$ Coulomb gases, we extend the orthogonal polynomials approach to extreme value statistics of random matrices from the Hermitian case [60] to non-Hermitian random matrices. Then we use this approach to show convergence of probability distribution of the rescaled eigenvalue with largest modulus of normal random matrices to Gumbel distribution, as well as universality of this result. This provides a simplified, alternative derivation of result in [98] and [99] for Coulomb gases. Next, we show that this approach can also be used to show convergence and universality of the distribution of the eigenvalue with smallest modulus at the inner edge of a ring distribution. We also discuss how the here presented approach can be used to obtain finite $N$ corrections of such extreme value statistics of normal random matrices and $2 D$ Coulomb gases. At various places the analytical results are complemented with numerical results.

This chapter is based on the following article:

"On the extreme value statistics of normal random matrice", $R$. Ebrahimi, S. Zohren, [arXiv: 1704.07488v1].

\section{1}

\section{EVS of Gaussian Random Normal Matrices at the Outer Edge}

In contrast to GUE, the eigenvalues of normal matrices are complex. As the eigenvalues can spread over the complex plane (within the support), they are less correlated than in the unitary case. This is reflected by the extreme value statistics. In the above example, considering the distribution of the eigenvalue with the largest modulus, it is known $[98,99]$ that the rescaled distribution is given by a Gumbel distribution. Recall that the Gumbel distribution is one of three families which describe the extreme value statistics of iid random variables (Weilbull, Gumbel, 
Frechet). Thus the behaviour is quite different to the GUE where the distribution of the largest eigenvalue is given by the Tracy-Widom distribution.

We recall that normal random matrices are defined through the measure ${ }^{1}$

$$
\mathbb{P}(M) \mathrm{d} M=\frac{1}{Z_{N}} e^{-\frac{N}{t} \operatorname{tr} V(M)} \mathrm{d} M, \quad t \in \mathbb{R}^{+},
$$

where $M^{\prime}$ 's are complex $N \times N$ matrices satisfying the constraint $\left[M, M^{\dagger}\right]=0$ and $Z_{N}$ is the partition function. They are in many senses similar to Wigner matrices with iid entries and in particular in the case of a Gaussian potential, yield the same eigenvalue density. However, for normal random matrices there exist a unitary matrix which simultaneously diagonalizes $M$ and $M^{\dagger}$. Thus we can employ Coulomb gas technique. In particular, the joint probability measure of the complex eigenvalues is given by

$$
P_{N}\left(z_{1}, \ldots, z_{N}\right)=\frac{1}{Z_{N}} \prod_{1 \leq j<k \leq N}\left(z_{j}-z_{k}\right)^{2} e^{-\frac{N}{t} \sum_{i=1}^{N} V\left(z_{i}\right)}, \quad t \in \mathbb{R}^{+} .
$$

For radially symmetric potentials

$$
V(z) \equiv V(r), \quad z=r e^{i \theta},
$$

we obtained the useful result in Ch. 7 that the monomials $\left(z^{n}\right)_{n \in \mathbb{N}_{0}}$ form a set of orthogonal polynomials with respect to inner product $\langle\cdot \mid \cdot\rangle_{\mu}$ with measure $\mu$, i.e.,

$$
\left\langle P_{n} \mid P_{m}\right\rangle_{\mu}:=\int \mathrm{d} \mu(z) P_{n}(z) \overline{P_{m}(z)}=h_{n} \delta_{n m}
$$

in which $\mathrm{d} \mu(z) \equiv e^{-\frac{N}{t} V(|z|)} \mathrm{d}^{2} z$, or more generally, $\mathrm{d} \mu(z ; y) \equiv e^{-\frac{N}{t} V(|z|)} \mathbb{I}_{\left\{|z| \zeta_{>}\right\}}$, in which case, $h_{n}=h_{n}(y)$.

For the Gaussian normal matrix ensemble, we know that the extreme value statistics is given by the Gumbel distribution. Therefore, we introduce monomials

$$
P_{n}(z ; y):=z^{n-1}
$$

for which the following orthogonality relation holds:

$$
\int \mathrm{d}^{2} z e^{-\frac{N}{t}|z|^{2}} \mathbb{I}_{\{|z| \leq y\}} P_{n}(z ; y) \overline{P_{m}(z ; y)}=h_{n}(y) \delta_{n m} .
$$

As the joint distribution of eigenvalues is given by (9-2), the cdf of eigenvalue with the largest modulus $\left|z_{\max }\right|$ reads (c.f. Sec. 8.1 and (8-14))

$$
F_{N}(y):=\mathbb{P}_{N}\left(\left|z_{\max }\right| \leq y\right)=\frac{Z_{N}(y)}{Z_{N}(\infty)},
$$

${ }^{1}$ The factor $t$ is usually introduced in order to consider multi critical potentials (see, e.g., [90]). Here, $t$ is merely introducet to simplify calculations. Later, in this chapter we will choose $t=2$. 
where the partition function

$$
Z_{N}(y)=\frac{1}{N !} \prod_{i=1}^{N} \int_{-\infty}^{y} \mathrm{~d} z_{i} \prod_{1 \leq j<k \leq N}\left(z_{j}-z_{k}\right)^{2} e^{-\frac{N}{t} \sum_{i=1}^{N} z_{i}^{2}}
$$

can be written as a product of the normalization constants $h_{n}$ :

$$
Z_{N}(y)=\prod_{n=0}^{N-1} h_{n}(y)
$$

in which

Therefore,

$$
h_{n}(y)=2 \pi \int_{0}^{y} \mathrm{~d} r r^{2 n+1} e^{-\frac{N}{t} r^{2}}
$$

$$
F_{N}(y)=\prod_{n=0}^{N-1} \frac{h_{n}(y)}{h_{n}(\infty)}
$$

We want to evaluate the above expression and scale it around the outer edge of the support which is at $|z|=\sqrt{t}$. In fact, once $N$ becomes large, the probability density $f_{N}(y)=F_{N}^{\prime}(y)$ will be more and more centered around the edge of the support, $c_{+}=\sqrt{t}$. More precisely, one expects $\left(\left|z_{\max }\right|-c_{+}\right) \sim N^{-1 / 2}$, thus to obtain convergence to a limiting distribution one has to rescale $y$ to a "continuum" variable $Y$,

$$
y=c_{+}+C N^{-1 / 2}[\Psi(N)+\Phi(N) Y]
$$

where $C$ is a constant and $\Psi(N)$ and $\Phi(N)$ are slowly varying functions (see App. A). The constant $C$ is chosen merely for convenience and could equally be absorbed in $\Psi(N)$ and $\Phi(N)$. Since $y \geq 0$, we have $Y \geq-\Psi(N) / \Phi(N)$. For the limiting distribution, to have support on $Y \in(-\infty,+\infty)$, we thus require $\Psi(N) / \Phi(N) \rightarrow \infty$ as $N \rightarrow \infty$. This is enough to obtain the extreme value statistics related to the eigenvalue with the largest modulus for the Gaussian potential. We are going to do this in the next two sections, using two slightly different approaches. Both approaches are based on the saddle point method. However, the first approach uses the asymptotics of regularized incomplete gamma function, while the second approach uses the asymptotics of error function.

\subsection{1}

\section{First Approach Using Asymptotics of Regularized Incomplete Gamma Function}

The starting point of this approach is to notice that in (9-11) one can write ${ }^{2}$

in which

$$
\frac{h_{n}(y)}{h_{n}(\infty)}=\frac{\int_{0}^{y} \mathrm{~d} r r^{2 n+1} e^{-N r^{2} / 2}}{\int_{0}^{\infty} \mathrm{d} r r^{2 n+1} e^{-N r^{2} / 2}}=\frac{\gamma\left(n+1, N y^{2} / 2\right)}{\Gamma(n+1)},
$$

\footnotetext{
${ }^{2}$ Here, we have chosen $t=2$.
} 
is the gamma function, and

$$
\Gamma(a):=\int_{0}^{\infty} \mathrm{d} u e^{-u} u^{a-1}, \quad \Re(a)>0
$$

$$
\gamma(a, z):=\int_{0}^{z} \mathrm{~d} u e^{-u} u^{a-1}, \quad \Re(a)>0
$$

is the lower incomplete gamma function. Inserting (9-13) into (9-11) and taking logarithm of both sides, we have

$$
\begin{aligned}
\ln \left(F_{N}(y)\right)=\sum_{n=0}^{N-1} \ln \left(\frac{h_{n}(y)}{h_{n}(\infty)}\right) & =\sum_{n=0}^{N-1} \ln \left(\frac{\gamma\left(n+1, N y^{2} / 2\right)}{\Gamma(n+1)}\right) \\
& =\sum_{n=1}^{N} \ln \left(\frac{\gamma\left(n, N y^{2} / 2\right)}{\Gamma(n)}\right)
\end{aligned}
$$

Therefore, in the large $N$ limit one has

$$
\lim _{N \rightarrow \infty} \ln \left(F_{N}(y)\right)=\lim _{N \rightarrow \infty} \sum_{n=1}^{N} \ln \left(P\left(n, N y^{2} / 2\right)\right)
$$

in which $P(a, z):=\frac{\gamma(a, z)}{\Gamma(a)}$ is the regularized lower incomplete gamma function. Next, we consider the scaling relation (9-12). We know that $\Psi(N)$ is a slowly varying function which diverges for $N \rightarrow \infty$. Furthermore, we require $\Psi(N) / \Phi(N) \rightarrow \infty$ as $N \rightarrow \infty$. It will become clear later that we will have to choose $\Phi(N) \sim 1 / \Psi(N)$ and moreover, that it will be convenient to choose $\Phi(N)=\frac{1}{2 \Psi(N)}$. The factor of 2 is merely a rescaling of $Y$ which could otherwise be determined at the end of the calculation. We thus have the scaling

$$
y=c_{+}+(N b)^{-1 / 2}\left[\Psi(N)+\frac{1}{2 \Psi(N)} Y\right]
$$

where $c_{+}=\sqrt{2}$, which yields

$$
\frac{N y^{2}}{2}=N+\frac{\Psi^{2}(N)}{2}+\sqrt{2 N} \Psi(N)+\left(\frac{\sqrt{N / 2}}{\Psi(N)}+\frac{1}{2}\right) Y+\mathcal{O}\left((\Psi(N))^{-2}\right) .
$$

Since the slowly varying function satisfies $\lim _{N \rightarrow \infty} \Psi(N)=+\infty$, one concludes that for all $y$ of the form given by (9-18), the following inequality holds:

$$
n<N y^{2} / 2
$$

Besides, one observes that ${ }^{3}$

$$
\lim _{N \rightarrow \infty} \ln \left(P\left(n, N y^{2} / 2\right)\right)=0, \quad \text { for } n \ll N y^{2} / 2 .
$$

Thus we can replace the summand in (9-17) by its asymptotics when both $N$ and $n$ are large. In fact, the asymptotic expansion of $P(a, z)$ for $a>0$ and $z \geq 0$ is given

${ }^{3}$ This has practical importance in the numerical simulations. 
by (see App. B):

$$
P(a, z) \sim \frac{1}{2} \operatorname{erfc}[-\operatorname{sgn}(\lambda-1) \sqrt{a} q(\lambda)], \quad \text { (uniformly for } z \geq 0 \text { ) }
$$

as $a \rightarrow \infty$ and $|\arg (a)|<\pi . q(\lambda)$ is given by

$$
q(\lambda):=\sqrt{\lambda-1-\ln \lambda},
$$

with $\lambda=z / a$. Thus one can write (9-17) as

$$
\lim _{N \rightarrow \infty} \ln \left(F_{N}(y)\right)=\lim _{N \rightarrow \infty} \sum_{n=1}^{N} \ln \left\{\frac{1}{2} \operatorname{erfc}\left[-\operatorname{sgn}\left(N y^{2} / 2 n-1\right) \sqrt{n} q\left(N y^{2} / 2 n\right)\right]\right\}
$$

Next, according to (9-20), the sign of the argument of the error function in (9-24) is easily determined:

$$
\operatorname{sgn}\left(N y^{2} / 2 n-1\right)=+1
$$

Using this fact, (9-24) simplifies to

$$
\lim _{N \rightarrow \infty} \ln \left(F_{N}(y)\right)=\lim _{N \rightarrow \infty} \sum_{n=1}^{N} \ln \left\{\frac{1}{2} \operatorname{erfc}\left[-\sqrt{n} q\left(N y^{2} / 2 n\right)\right]\right\} .
$$

When both $n$ and $N$ are large while $n / N=: \xi \in[0,1]$, we can obtain the first two leading order terms in (9-26) using Euler-MacLaurin summation formula (see App. C):

$$
\lim _{N \rightarrow \infty} \ln \left(F_{N}(y)\right)=\lim _{N \rightarrow \infty} N \int_{0}^{1} \mathrm{~d} \xi g(\xi ; y)+\frac{1}{2}(g(1 ; y)-g(0 ; y))+\mathcal{O}\left(N^{-1}\right)
$$

in which

$$
g(\xi ; y) \equiv \ln \left\{\frac{1}{2} \operatorname{erfc}\left[-\sqrt{N \xi} q\left(y^{2} / 2 \xi\right)\right]\right\} .
$$

For the argument of the error function in (9-28), one has

$$
\sqrt{N \xi} q\left(y^{2} / 2 \xi\right)= \begin{cases}\mathcal{O}(\sqrt{N}), & \xi \in[0,1) \\ \mathcal{O}(\sqrt{\Psi(N)}), & \xi=1\end{cases}
$$

Therefore, in the limit $N \rightarrow \infty$, one can use the asymptotics of the complementary error function for large negative values of its argument:

$$
\operatorname{erfc}(z) \equiv \frac{2}{\sqrt{\pi}} \int_{z}^{\infty} \mathrm{d} u e^{-u^{2}} \sim 2+\frac{e^{-z^{2}}}{\sqrt{\pi} z}, \quad z \rightarrow \infty \quad(\Re(z)<0) .
$$

Hence, one obtains 


$$
\begin{aligned}
g(\xi ; y) & \sim \ln \left\{\frac{1}{2}\left[2-\frac{e^{-N \xi q^{2}\left(y^{2} / 2 \xi\right)}}{\sqrt{\pi} \sqrt{N \xi} q\left(y^{2} / 2 \xi\right)}\right]\right\}, \quad N \rightarrow \infty \\
& \sim-\frac{1}{2 \sqrt{\pi}} \frac{e^{-N \xi q^{2}\left(y^{2} / 2 \xi\right)}}{\sqrt{N \xi} q\left(y^{2} / 2 \xi\right)}, \quad N \rightarrow \infty
\end{aligned}
$$

Therefore, the leading order in (9-27) reads

$$
\lim _{N \rightarrow \infty} \ln \left(F_{N}(y)\right)=\lim _{N \rightarrow \infty}-\frac{N}{2 \sqrt{\pi}} \int_{0}^{1} \mathrm{~d} \xi \frac{e^{-N \xi q^{2}\left(y^{2} / 2 \xi\right)}}{\sqrt{N \xi} q\left(y^{2} / 2 \xi\right)}
$$

Now, we can apply the saddle point method to the last integral: the function $q(\lambda)$ attains its minimum at $\lambda_{0}=1$, for which

$$
\begin{aligned}
q^{2}\left(\lambda_{0}\right) & =0 \\
\left.\frac{\mathrm{d}}{\mathrm{d} \lambda} q^{2}(\lambda)\right|_{\lambda=\lambda_{0}} & =0 \\
\left.\frac{\mathrm{d}^{2}}{\mathrm{~d} \lambda^{2}} q^{2}(\lambda)\right|_{\lambda=\lambda_{0}} & =1
\end{aligned}
$$

Thus the expansion of $q^{2}(\lambda)$ around its minimum takes the form

$$
\begin{aligned}
q^{2}(\lambda) & =\frac{1}{2}\left(\left.\frac{\mathrm{d}^{2}}{\mathrm{~d} \lambda^{2}} q^{2}(\lambda)\right|_{\lambda=\lambda_{0}}\right)\left(\lambda-\lambda_{0}\right)^{2}+\mathcal{O}\left(\left(\lambda-\lambda_{0}\right)^{3}\right) \\
& =\frac{1}{2}(\lambda-1)^{2}+\mathcal{O}\left((\lambda-1)^{3}\right)
\end{aligned}
$$

Inserting this result into (9-32), we obtain

$$
\lim _{N \rightarrow \infty} \ln \left(F_{N}(y)\right)=\lim _{N \rightarrow \infty}-\frac{N}{2 \sqrt{\pi}} \int_{0}^{1} \mathrm{~d} \xi \frac{e^{-\frac{N}{2 \xi}\left(y^{2} / 2-\xi\right)^{2}}}{\sqrt{\frac{N}{2 \xi}}\left(y^{2} / 2-\xi\right)} .
$$

The main contribution to the integral in (9-35) comes from the terms with $\xi$ close to one. this is equivalent to the fact that according to (9-21), only those terms with $n \approx N$ make significant contribution to the summation in (9-17). Hence, we introduce the following change of variable in the integration in (9-35):

$$
\xi=: 1-\frac{(2 N)^{-1 / 2}}{\Psi(N)} X .
$$

Now, we apply the scaling (9-18) and change the integration variable as given in (9-36). Then using

$$
\xi^{ \pm 1 / 2}=\left[1-\frac{(2 N)^{-1 / 2}}{\Psi(N)} X\right]^{ \pm 1 / 2}=1 \pm \frac{(2 N)^{-1 / 2}}{2 \Psi(N)} X+\mathcal{O}\left(N^{-1}(\Psi(N))^{-2}\right)
$$

one can show 


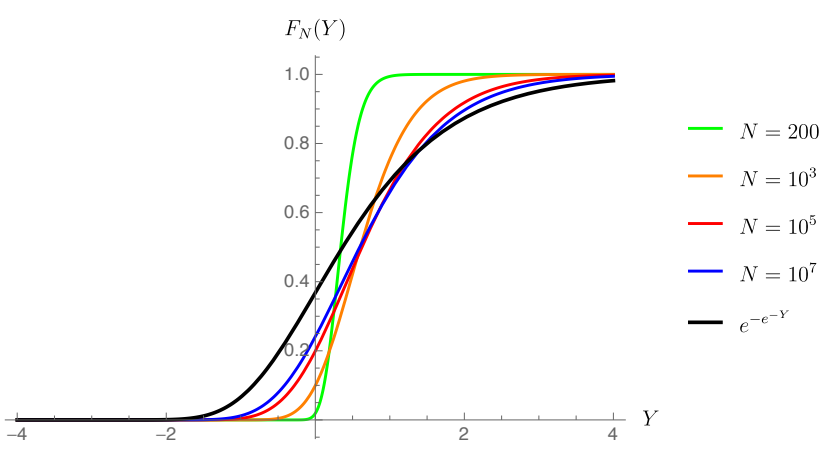

Figure 9.1: Shown is the function $F_{N}(Y)$ for increasing values of $N$, as well as the limiting case $F(Y)=\exp (-\exp (-Y))$. The function $F_{N}(Y)$ is obtained by numerically evaluating (9-11) with $y$ replaced with its scaling relation $(9-18)$.

$$
\sqrt{\frac{N}{2 \xi}}\left(\frac{y^{2}}{2}-\xi\right)=\Psi(N)+\frac{1}{2 \Psi(N)}(X+Y)+\mathcal{O}\left(N^{-1 / 2}(\Psi(N))^{-2}\right) .
$$

This relation also justifies the above choices of scalings. In particular, we notice that

$$
\frac{N}{2 \xi}\left(\frac{y^{2}}{2}-\xi\right)^{2}=\Psi^{2}(N)+(X+Y)+\mathcal{O}\left(N^{-1 / 2}(\Psi(N))^{-1}\right)
$$

is a slowly varying function plus a finite term. To get this, it was necessary to choose $\Phi(N) \sim 1 / \Psi(N)$. Putting everything together, we get

$$
\begin{aligned}
\lim _{N \rightarrow \infty} \ln \left(F_{N}(Y)\right) & =\lim _{N \rightarrow \infty}-\frac{\sqrt{N} e^{-\Psi^{2}(N)}}{2 \sqrt{2 \pi} \Psi^{2}(N)}\left(\int_{0}^{\Psi(N) \sqrt{2 N}} \mathrm{~d} X e^{-X}\right) e^{-Y} \\
& =\lim _{N \rightarrow \infty}-\frac{\sqrt{N} e^{-\Psi^{2}(N)}}{2 \sqrt{2 \pi} \Psi^{2}(N)} e^{-Y}
\end{aligned}
$$

Now, if we make the following choice for the slowly varying function $\Psi(N)$ :

$$
\Psi^{2}(N)=\frac{1}{2}(\ln N-2 \ln (\ln N)-\ln (2 \pi)),
$$

we arrive at

$$
N \int_{0}^{1} \mathrm{~d} \xi g(\xi ; y)=-e^{-Y}+\mathcal{O}(\ln (\ln N) / \ln N) .
$$

Furthermore, under this choice, for the subleading term on the right hand side of (9-27) we have

$$
\frac{1}{2}(g(1 ; y)-g(0 ; y))=\mathcal{O}\left((\Psi(N))^{-1} e^{-\Psi^{2}(N)}\right)=\mathcal{O}(\sqrt{\ln N / N}) .
$$

The final result is thus given by 

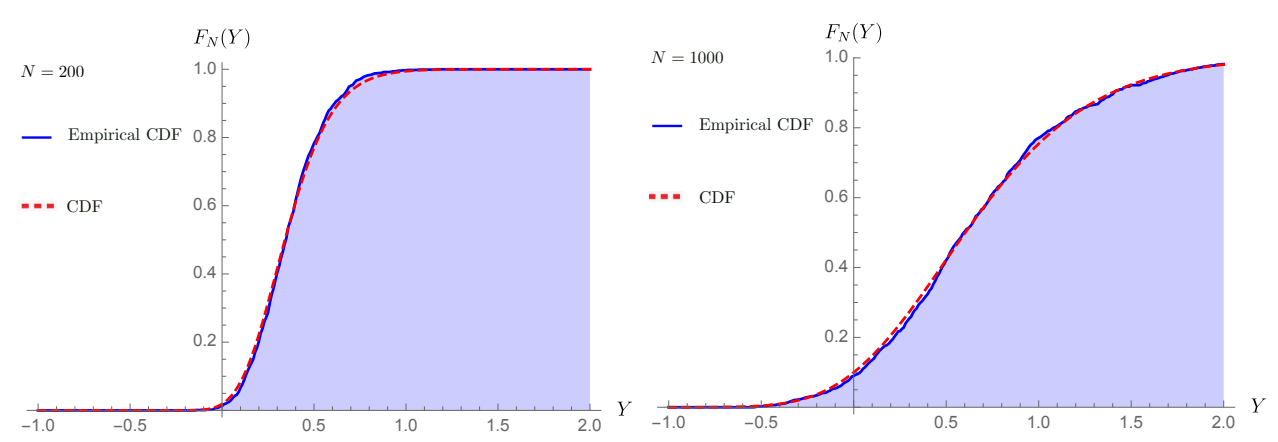

Figure 9.2: Comparison of the finite $N$ expression $F_{N}(Y)$ as given in (9-13) with the empirical cumulative probability function obtained from sampling 1000 Ginibre matrices of size $N=200$ and $N=1000$ respectively.

$$
\begin{aligned}
F(Y) & :=\lim _{N \rightarrow \infty} F_{N}(Y) \\
& =\lim _{N \rightarrow \infty} \mathbb{P}_{N}\left(\left|z_{\max }\right| \leq c_{+}+(N b)^{-1 / 2}\left[\Psi(N)+\frac{Y}{2 \Psi(N)}\right]\right)=e^{-e^{-Y},(9-44)}
\end{aligned}
$$

which is precisely the Gumbel distribution. This provides an alternative derivation of the results in [98].

One strength of the here presented approach is that it is particularly suited for calculating finte $N$ corrections of the limiting distribution $\lim _{N \rightarrow \infty} F_{N}(Y)=F(Y)=\exp (-\exp (-Y))$. This can be important in practice, since the convergence to the Gumbel distribution is very slow as can be seen from the order of the subleading term in (9-42).

Figure 9.1 shows $F_{N}(Y)$ evaluated for increasing values of $N$. It is clear that for any practical applications, the limiting distribution is not a good approximation. However, the result presented in (9-16), provides a closed expression for $F_{N}(Y)$ for finite $N$, which can easily be evaluated numerically by inserting the slowly varying function (9-41) into (9-16), as had been done in Figure 9.1. For large values of $N$, one can benefit from the fact that the sum in (9-16) is dominated by $n \approx N$. We can also verify the correctness of this expression by comparing the result of $F_{N}(Y)$ with the empirical cumulative probability function obtained from sampling $m$ representations of a Ginibre matrix of size $N$. This is shown in Figure 9.2 for $m=1000$, with $N=200$ and $N=1000$ respectively.

Note that in principle the here presented techniques can also be used to compute the next-to-leading order term in (9-42) and (9-44). 


\subsection{2}

\section{Second Approach Using Asymptotics of Error Function}

The starting point of this approach is applying saddle point evaluation from the beginning in (9-10). In fact, we can write (9-10) as

$$
h_{n}(y)=2 \pi \int_{0}^{y} \mathrm{~d} r e^{N f(r)}
$$

with $f(r)=(2 n+1) / N \ln r-r^{2} / t$. Then, we expand $f$ around its maximum at $r_{0}=\sqrt{(2 n+1) t / 2 N}$ as

$$
f(r)=f\left(r_{0}\right)-\frac{1}{2}\left|f^{\prime \prime}\left(r_{0}\right)\right|\left(r-r_{0}\right)^{2}+\mathcal{O}\left(\left(r-r_{0}\right)^{3}\right) .
$$

The integration in (9-10) then reduces to Gaussian integrals over finite intervals, yielding error functions:

$$
\frac{h_{n}(y)}{h_{n}(\infty)}=\frac{\operatorname{erf}\left(\sqrt{N b} r_{0}\right)+\operatorname{erf}\left(\sqrt{N b}\left(y-r_{0}\right)\right)}{1+\operatorname{erf}\left(\sqrt{N b} r_{0}\right)}
$$

where $b \equiv\left|f^{\prime \prime}\left(r_{0}\right) / 2\right|=2 / t$. Now using Euler-Maclaurin summation formula, one has

$$
\ln \left(F_{N}(y)\right)=N \int_{0}^{1} \mathrm{~d} \xi g(\xi ; y)+\frac{1}{2}(g(1 ; y)-g(0 ; y))+\mathcal{O}\left(N^{-1}\right)
$$

where

$$
g(\xi ; y) \equiv \ln \left(\frac{\operatorname{erf}\left(\sqrt{N b} r_{0}(\xi)\right)+\operatorname{erf}\left(\sqrt{N b}\left(y-r_{0}(\xi)\right)\right)}{1+\operatorname{erf}\left(\sqrt{N b} r_{0}(\xi)\right)}\right)
$$

in which $r_{0}(\xi)=\sqrt{t \xi}+\mathcal{O}\left(N^{-1}\right)$.

Note that the argument of the first error function in the denominator of (9-49) is not necessarily large if $\xi$ is close to zero. However, the asymptotics of (9-49) is dominated by the second term. One then can use the asymptotic expansion of the error function

$$
\operatorname{erf}(z) \equiv \frac{2}{\sqrt{\pi}} \int_{0}^{z} \mathrm{~d} u e^{-u^{2}} \sim 1-\frac{e^{-z^{2}}}{\sqrt{\pi} z}, \quad z \rightarrow \infty \quad(\Re(z)>0),
$$

to show

$$
g(\xi ; y) \sim-\frac{1}{\sqrt{\pi}} \frac{e^{-N b\left(y-r_{0}(\xi)\right)^{2}}}{\sqrt{N b}\left(y-r_{0}(\xi)\right)\left(1+\operatorname{erf}\left(\sqrt{N b} r_{0}(\xi)\right)\right)}, \quad N \rightarrow \infty
$$

Now, since $\lim _{N \rightarrow \infty} \Psi(N)=\infty$, the scaling (9-18) ensures that the argument of the second error function, $\sqrt{\mathrm{Nb}}\left(y-r_{0}(\xi)\right)$, tends to infinity as $N \rightarrow \infty$. Furthermore, since $r_{0}(\xi) \rightarrow c_{+}$as $\xi \rightarrow 1$, the dominant contribution in (9-51) comes from $\xi$ close 
to one, in which case we have

$$
g(\xi ; y) \sim-\frac{1}{2 \sqrt{\pi}} \frac{e^{-N b\left(y-r_{0}(\xi)\right)^{2}}}{\sqrt{N b}\left(y-r_{0}(\xi)\right)}, \quad N \rightarrow \infty .
$$

Therefore, for the leading term in the right hand sight of (9-48) we have

$$
N \int_{0}^{1} \mathrm{~d} \xi g(\xi ; y) \sim-\frac{N}{2 \sqrt{\pi}} \int_{0}^{1} \mathrm{~d} \xi \frac{e^{-N b\left(y-r_{0}(\xi)\right)^{2}}}{\sqrt{N b}\left(y-r_{0}(\xi)\right)} .
$$

Next, by applying (9-18) and (9-36) in the above expression, we obtain

$$
\begin{aligned}
N \int_{0}^{1} \mathrm{~d} \xi g(\xi ; y) & =-\frac{\sqrt{N}}{2 \sqrt{2 \pi} \Psi^{2}(N)} \int_{0}^{\sqrt{2 N} \Psi(N)} \mathrm{d} X e^{-\left[\Psi^{2}(N)+(X+Y)\right]} \\
& =-\frac{\sqrt{N} e^{-\Psi^{2}(N)}}{2 \sqrt{2 \pi} \Psi^{2}(N)} e^{-Y}
\end{aligned}
$$

Then by choosing the form given in (9-41) for slowly varying function $\Psi(N)$, we retrieve the Gumbel distribution:

$$
\lim _{N \rightarrow \infty} \mathbb{P}_{N}\left(\left|z_{\max }\right| \leq c_{+}+(N b)^{-1 / 2}\left[\Psi(N)+\frac{Y}{2 \Psi(N)}\right]\right)=e^{-e^{-Y}}
$$

\section{2}

\section{Universality of Extreme Value Statistics}

In the following next two sections, we discuss the universality of EVS obtained for the Gaussian random normal matrices, for an arbitrary potential with radial symmetry satisfying (7-24), together with another condition which will be derived in he next section, at finite inner and outer edges. It turns out that the same general procedure used for the Gaussian potential can be applied with a slight modification.

\subsection{1}

\section{Universality of EVS at Finite Outer Edge}

Next, we consider generalisation of the Gaussian potential to an arbitrary potential $V$ with radial symmetry, $V \equiv V(r)$. We then have

$$
h_{n}(y)=2 \pi \int_{0}^{y} \mathrm{~d} r r^{2 n+1} e^{-\frac{N}{t} V(r)}=\int_{0}^{y} \mathrm{~d} r e^{N f(r)},
$$

where $f(r)=(2 n+1) / N \log r-V(r) / t$ with a maximum at $r_{0}=r_{0}(\xi)$ which is solution to following equation

$$
r_{0}(\xi) V^{\prime}\left(r_{0}(\xi)\right)=\frac{2 n+1}{N} t=2 t \xi+\mathcal{O}\left(N^{-1}\right) .
$$


Comparing (7-29) with (9-57) mplies that in the large $N$ limit, the outer edge and $r_{0}$ coincide, i.e., $r_{0}(1)=c_{+}$.

Next, we expand $f$ around its maximum at $r_{0}$ :

$$
f(r)=f\left(r_{0}\right)+\frac{f^{\prime \prime}\left(r_{0}\right)}{2}\left(r-r_{0}\right)^{2}+\mathcal{O}\left(\left(r-r_{0}\right)^{3}\right)
$$

in which in the large $N$ limit

$$
\begin{aligned}
f^{\prime \prime}\left(r_{0}\right) & =-2 \xi / r_{0}^{2}+V^{\prime \prime}\left(r_{0}\right) / t \\
& =-\frac{1}{t}\left(\frac{V^{\prime}\left(r_{0}\right)}{r_{0}}+V^{\prime \prime}\left(r_{0}\right)\right) \\
& =-\left.\left(\frac{1}{\operatorname{tr}} \frac{\mathrm{d}}{\mathrm{d} r}\left(r V^{\prime}(r)\right)\right)\right|_{r=r_{0}},
\end{aligned}
$$

where in going from the second line to the third line, we have used (9-57). Therefore $f^{\prime \prime}\left(r_{0}\right)<0$, if the condition

$$
r V^{\prime}(r) \text { increasing in } \mathbb{R}^{+}
$$

or equivalently

$$
V^{\prime}(r)>0 \text { and } V \text { convex in } \mathbb{R}^{+}
$$

holds for the potential $V(r)$. Hence, as in the case of Gaussian potential, we have (see $(9-47))$

$$
\frac{h_{n}(y)}{h_{n}(\infty)}=\frac{\operatorname{erf}\left(\sqrt{N b} r_{0}\right)+\operatorname{erf}\left(\sqrt{N b}\left(y-r_{0}\right)\right)}{1+\operatorname{erf}\left(\sqrt{N b} r_{0}\right)}
$$

where $b \equiv\left|f^{\prime \prime}\left(r_{0}\right) / 2\right|$. Therefore, the leading order term in the probability distribution of the eigenvalue with the largest modulus is given by

$$
N \int_{0}^{1} \mathrm{~d} \xi g(\xi ; y) \sim-\frac{N}{2 \sqrt{\pi}} \int_{0}^{1} \mathrm{~d} \xi \frac{e^{-N b\left(y-r_{0}(\xi)\right)^{2}}}{\sqrt{N b}\left(y-r_{0}(\xi)\right)}, \quad N \rightarrow \infty .
$$

In (9-63) we scale $y$ at the outer edge of the support as before (see (9-18)). We also change the integration variable $\xi$ in (9-63) as

$$
\xi=1-\gamma_{+} \frac{(N b)^{-1 / 2}}{2 \Psi(N)} X
$$

The value of $\gamma_{+}$will be determined in the following. We expand $r_{0}(\xi)$ around $\xi=1$ : 


$$
\begin{aligned}
r_{0}(\xi) & =r_{0}(1)+\mathrm{d} r_{0}(\xi) /\left.\mathrm{d} \xi\right|_{\xi=1}(\xi-1)+\mathcal{O}\left((\xi-1)^{2}\right) \\
& =c_{+}+\delta_{+}(\xi-1)+\mathcal{O}\left((\xi-1)^{2}\right)
\end{aligned}
$$

where $\delta_{+}$is given by

$$
\begin{aligned}
\delta_{+} \equiv \mathrm{d} r_{0}(\xi) /\left.\mathrm{d} \xi\right|_{\xi=1} & =\frac{2 t}{V^{\prime}\left(c_{+}\right)+c_{+} V^{\prime \prime}\left(c_{+}\right)} \\
& =2 t\left(\left.\frac{\mathrm{d}}{\mathrm{d} r}\left(r V^{\prime}(r)\right)\right|_{r=c_{+}}\right)^{-1}
\end{aligned}
$$

Hence, according to the constraint already imposed in (9-60), we have $\delta_{+}>0$. We therefore obtain

$$
\sqrt{N b}\left(y-r_{0}(\xi)\right)=\Psi(N)+\frac{1}{2 \Psi(N)}\left(\gamma_{+} \delta_{+} X+Y\right)+\mathcal{O}\left(N^{-1 / 2}(\Psi(N))^{-2}\right)
$$

which by choosing

$$
\gamma_{+}=\frac{1}{\delta_{+}}=\frac{V^{\prime}\left(c_{+}\right)+c_{+} V^{\prime \prime}\left(c_{+}\right)}{2 t}=\left.\frac{1}{2 t} \frac{\mathrm{d}}{\mathrm{d} r}\left(r V^{\prime}(r)\right)\right|_{r=c_{+}}
$$

reduces to

$$
\sqrt{N b}\left(y-r_{0}(\xi)\right)=\Psi(N)+\frac{1}{2 \Psi(N)}(X+Y)+\mathcal{O}\left(N^{-1 / 2}(\Psi(N))^{-2}\right) .
$$

The rest of the derivation is identical to the Gaussian case presented in the previous section. We have thus shown universality of the distribution of the eigenvalue with the largest modulus when rescaled around the outer edge of a generic potential $V(r)$ satisfying the condition given in (9-60) or (9-61), where the limiting probability distribution is the Gumbel distribution; i.e., the probability distribution is a Gumbel distribution:

$$
\lim _{N \rightarrow \infty} \mathbb{P}_{N}\left(\left|z_{\max }\right| \leq c_{+}+(N b)^{-1 / 2}\left[\Psi(N)+\frac{Y}{2 \Psi(N)}\right]\right)=e^{-e^{-Y}}
$$

\subsection{2}

\section{Universality of EVS at Finite Inner Edge}

Now we study the extreme value statistics of a normal matrix ensemble with potential $V=V(r)$ at the inner edge. Again we can define monomials

$$
P_{n}(z ; y):=z^{n-1}
$$

which satisfy the following orthogonality condition 


$$
\int \mathrm{d}^{2} z e^{-\frac{N}{t} V(|z|)} \mathbb{I}_{\{|z| \geq y\}} p_{n}(z ; y) \overline{p_{m}(z ; y)}=h_{n}(y) \delta_{n m}, \quad t \in \mathbb{R}^{+} .
$$

The probability distribution for the eigenvalue with the smallest modulus $\left|z_{\min }\right|$ is then given by

$$
F_{N}(y):=\mathbb{P}\left(\left|z_{\min }\right| \geq y\right)=\frac{Z_{N}(y)}{Z_{N}(0)}=\prod_{n=0}^{N-1} \frac{h_{n}(y)}{h_{n}(0)} .
$$

We want to evaluate the above expression and scale it around the finite inner edge of the support at $|z|=c_{-}>0$. We have

$$
h_{n}(y)=2 \pi \int_{y}^{\infty} \mathrm{d} r r^{2 n+1} e^{-\frac{N}{t} V(r)}=2 \pi \int_{y}^{\infty} \mathrm{d} r e^{N f(r)}
$$

with $f(r)=(2 n+1) / N \log r-V(r) / t$. Then expanding $f$ around its maximum at $r_{0}$ as (9-57), results in

$$
\frac{h_{n}(y)}{h_{n}(0)}=\frac{1+\operatorname{erf}\left(\sqrt{N b}\left(r_{0}-y\right)\right)}{1+\operatorname{erf}\left(\sqrt{N b} r_{0}\right)}
$$

where $b \equiv\left|f^{\prime \prime}\left(r_{0}\right) / 2\right|$. Thus in the large $N$ limit one has

$$
\ln \left(F_{N}(y)\right)=N \int_{0}^{1} \mathrm{~d} \xi g(\xi ; y)+\frac{1}{2}(g(1 ; y)-g(0 ; y))+\mathcal{O}\left(N^{-1}\right),
$$

where

$$
g(\xi ; y) \equiv \ln \left(\frac{1+\operatorname{erf}\left(\sqrt{N b}\left(r_{0}(\xi)-y\right)\right)}{1+\operatorname{erf}\left(\sqrt{N b} r_{0}(\xi)\right)}\right)
$$

Since both $r_{0}$ and $r_{0}-y$ are positive and become large when $N$ is large, by recalling the asymptotic behaviour of the error function (see (9-50)), we obtain ${ }^{4}$

$$
N \int_{0}^{1} \mathrm{~d} \xi g(\xi ; y) \sim-\frac{N}{2 \sqrt{\pi}} \int_{0}^{1} \mathrm{~d} \xi \frac{e^{-N b\left(r_{0}(\xi)-y\right)^{2}}}{\sqrt{N b}\left(r_{0}(\xi)-y\right)}, \quad N \rightarrow \infty .
$$

Now, we scale $y$ around the inner edge of the support, $c_{-}$, as

$$
y=c_{-}-(N b)^{-1 / 2}\left[\Psi(N)+\frac{1}{2 \Psi(N)} Y\right], \quad Y \in(-\infty, \infty) .
$$

Note that as $N$ becomes large, $y$ approaches $c_{-}$. Moreover, $r_{0}(\xi)$ with $\xi=0$ also approaches $c_{-}$as $N$ becomes large, as is clear when comparing (7-28) with (9-57). This implies that the leading order contribution of (9-78) now comes from $\xi=0$. This suggests the following change of variables in (9-78)

$$
\xi=\gamma_{-} \frac{(N b)^{-1 / 2}}{2 \Psi(N)} X
$$

${ }^{4}$ In fact, the argument is now even simpler since $r_{0}(\xi)$ stays finite and does not go to zero as $\xi$ goes to zero. This is assured by the requirement that $c_{-}>0$. 
Now, expanding $r_{0}(\xi)$ around $\xi=0$ gives

$$
\begin{aligned}
r_{0}(\xi) & =r_{0}(0)+\mathrm{d} r_{0}(\xi) /\left.\mathrm{d} \xi\right|_{\xi=0} \xi+\mathcal{O}\left(\xi^{2}\right) \\
& =c_{-}+\delta_{-} \xi+\mathcal{O}\left(\xi^{2}\right)
\end{aligned}
$$

where

$$
\delta_{-} \equiv \mathrm{d} r_{0}(\xi) /\left.\mathrm{d} \xi\right|_{\xi=0}=\frac{2 t}{c_{-} V^{\prime \prime}\left(c_{-}\right)} .
$$

We notice that since $c_{-}$is finite and the potential $V$ is convex, we have $\delta_{-}>0$. We therefore obtain

$$
\sqrt{N b}\left(r_{0}(\xi)-y\right)=\Psi(N)+\frac{1}{2 \Psi(N)}\left(\gamma_{-} \delta_{-} X+Y\right)+\mathcal{O}\left(N^{-1 / 2}(\Psi(N))^{-2}\right)
$$

which by choosing

$$
\gamma_{-}=\frac{1}{\delta_{-}}=\frac{c_{-} V^{\prime \prime}\left(c_{-}\right)}{2 t}
$$

becomes

$$
\sqrt{N b}\left(r_{0}(\xi)-y\right)=\Psi(N)+\frac{1}{2 \Psi(N)}(X+Y)+\mathcal{O}\left(N^{-1 / 2}(\Psi(N))^{-2}\right) .
$$

Following the reasoning from the previous section we thus get

$$
\lim _{N \rightarrow \infty} \mathbb{P}_{N}\left(\left|z_{\min }\right| \geq c_{-}-(N b)^{-1 / 2}\left[\Psi(N)+\frac{Y}{2 \Psi(N)}\right]\right)=e^{-e^{-Y}} .
$$

where $\Psi(N)$ is given in (9-41). This shows universality by proving convergence of the rescaled distribution of the eigenvalue with smallest modulus to a Gumbel distribution. The derivation assumes that the potential fulfills the condition (9-60) or (9-61) and that the inner radius $c_{-}>0$, i.e., the support of the eigenvalue density has topology of a ring.

As a final point, important to be emphasized, is the fact that although the (complex) eigenvalues of a normal matrix ensemble with arbitrary potential $V(r)$ are correlated, due to the presence of the Vandermonde determinant in the jpdf of eigenvalues in (9-2), the EVS of the eigenvalue with the smallest or largest modulus is still given by Gumbel distribution. This implies that unlike the case of Hermitian random matrices, where the eigenvalues are strongly correlated, the eigenvalues of a random normal matrix ensemble are weakly correlated. 


\section{Outlook and Conclusion}

In this thesis, we discussed the EVS of random normal matrices. In the first part, we reviewed the necessary probabilistic aspects upon which the rest of this thesis was founded. While the first chapter began by briefly introducing the basics of probability theory, the second chapter dealt with the asymptotic models of classical EVS: first, we gave the motivation how these models of a sample of iid random variables naturally arise in parallel with the classical statistics, i.e., the central limit theory. The basic result of this chapter was mainly on the extermal types theorem according which there exist only three different universal classes of extreme value distributions for a sequence of iid random variables, under specific conditions. The necessary and sufficient conditions were then mentioned without proof.

The foundations of RMT were reviewd in part II. This was done basically by first, motivating RMT as a powerful phenomenological models in a variety areas of physics and mathematics. Then, the three classical ensembles of RMT, i.e., GUE, GOE and GSE were introduced. Next, the reason why random matrices are omnipresent in many fields of mathematics and physics, namely universality, was explained briefly. Then level spacing distribution as an example of such universal quantities was discussed.

In the final part of this thesis, our focus was on the applications of extreme value statistics in the context of RMT. In Ch. 8 we showed how one can derive the celebrated Tracty-Widom law for the extreme value statistics of GUE using Lax method which makes connection to integrable systems. Ch. 9 contained the new result of this thesis on the extreme value statistics of random normal matrices. For non-Hermitian matrices the situation is somewhat easier as eigenvalues are only weakly correlated and the extreme value statistics is given by the much simpler Gumbel distribution. However, due to the lack of symmetries, non-Hermitian random matrices are in general harder to deal with. A special case are normal random matrices which are non-Hermitian and at the same time allow for a Coulomb gas formulation for a generic potential. 
The novel contribution in this thesis was presented in Ch. 9, where we investigated the extreme value statistics of normal random matrices and $2 D$ Coulomb gases for general radially symmetric potentials. This was done by extending the orthogonal polynomial approach, introduced in [60] for Hermitian matrices, to normal random matrices and $2 D$ Coulomb gases. We first analysed the simplest case of Gaussian normal random matrices and showed convergence of the eigenvalue with largest modulus, rescaled around the outer edge of the eigenvalue support, to a Gumbel distribution. One strength of this approach lies in the fact that it immediately generalises to an arbitrary potential $V=V(r)$ with radial symmetry which meets some conditions. We used this to show universality of the distribution of the eigenvalue with largest modulus when rescaled around the outer edge of the eigenvalue support. This provides an alternative, simplified derivation of results presented in $[98,99]$. In addition, it was shown that the approach presented here also generalised to compute convergence of the distribution of the eigenvalue with smallest modulus rescaled around the inner edge of the eigenvalue support, with topology of an annulus.

The here presented approach can also be used to obtain finite $N$ results. Firstly, for Ginibre matrices, the expression (9-17) can easily be evaluated numerically for finite $N$. This was done in the comparison presented in Figure 9.2, where we plotted the numerically evaluated cumulative probability function (9-17) against the empirical cumulative probability function obtained from samples of Ginibre matrices for finite $N$. Secondly, the expansion (9-48), and the analogous expression for general potential can, in principle, be used to obtain an analytical expression of the next-to-leading-order term. We leave the details of such an analysis for future work. Note that this calculation follows closely the standard finite $N$ expansion of the free energy of Hermitian random matrices using orthogonal polynomials (see for example Section 2.3 of [53]).

Another interesting direction of future research is to explore the extreme value statistics of the eigenvalue with smallest modulus at the the transition, when the inner radius of the eigenvalue support goes to zero as $N$ goes to infinity. This transition was analysed in [83] in the context of the mean radial displacement. It would be interesting to see whether the extreme value statistics can be tuned to a different universality class in this transition by using a double scaling limit in which $a_{-}$goes to zero as $N$ becomes large. In fact, one can show that in this case, the expression (9-73) can be expressed in terms of regularized upper incomplete gamma function. Then by applying the asymptotic expansion of the regularized upper incomplete gamma function, given in App. B, one may find the EVS of a 
normal matrix ensemble when inner edge is zero.

The other interesting case is when the outer edge of the support is not finite. An example is Cauchy distribution with potential $V(r)=\ln \left(1+r^{2}\right)$ which does not satisfy the condition (7-24). In this case, one can easily check that the expression (9-7) can be written in terms of regularized incomplete beta function. Hence, by applying suitable asymptotic expansion of beta function, one can, in principle, find the EVS of Cauchy normal ensemble at the infinite outer edge. 


\section{A \\ Regularly and Slowly Varying Functions}

Regular variation is one of the basic concepts which appears in a natural way in different contexts of applied probability theory. The concept of regularly varying function was proposed by Jovan Karamata [100], and roughly speaking, are those functions which behave asymptotically like power functions. In this appendix, we briefly review the basic definitions and theorems of Karamata's theory. Some references are $[17,37,101,102]$.

Suppose $x_{0} \in \mathbb{R}^{+}$and let $f:\left[x_{0}, \infty\right) \longrightarrow \mathbb{R}^{+}$be a measurable function, ${ }^{1}$ such that the limit

$$
\lim _{x \rightarrow \infty} \frac{f(t x)}{f(x)}
$$

exists for all $t \in \mathbb{R}^{+}$. Then there exists $\rho \in \mathbb{R}$ such that

$$
\lim _{x \rightarrow \infty} \frac{f(t x)}{f(x)}=t^{\rho}
$$

for all $t \in \mathbb{R}^{+}$. Then depending on the value of $\rho$, we distinguish the following classes of functions: ${ }^{2}$

- If $\rho \neq 0$, the function $f$ is said to be regularly varying at infinity with index $\rho$, denoted $f \in \mathcal{R} \mathcal{V}_{\infty}(\rho)$.

- If $\rho=0$, the function $f$ is said to be slowly varying at infinity, denoted $f \in \mathcal{R} \mathcal{V}_{\infty}(0)$.

As a result of this classification, we have Karamata's characterization theorem:

Let $\rho \neq 0$ and $f \in \mathcal{R} \mathcal{V}_{\infty}(\rho)$; then there exists a slowly varying function $\Psi$, such that $f(x)=x^{\rho} \Psi(x)$.

The properties of regularly varying functions are thus deduced from those of slowly varying functions and vice versa.

\footnotetext{
${ }^{1}$ The measurability assumption is essential.

${ }^{2} \mathrm{~A}$ third class of rapidly varying functions can be considered if we let $\rho \in \overline{\mathbb{R}}$; i.e., if $\rho= \pm \infty$. In this way, the class of regularly varying functions "fills the gap" between the class of slowly varying functions and the class of rapidly varying functions.
} 
Finally, we state the Karamata representation theorem:

A function $\Psi$ is slowly varying iff there exists $x_{0}>0$ such that the function $\Psi$ can be written in the form

$$
\Psi(x)=\exp \left(\eta(x)+\int_{x_{0}}^{x} \mathrm{~d} t \frac{\epsilon(t)}{t}\right), \quad \forall x \geq x_{0},
$$

where $\eta(x)$ and $\epsilon(x)$ are bounded measurable functions of a real variable $x$, such that

$$
\lim _{x \rightarrow \infty} \eta(x)<\infty, \quad \text { and } \quad \lim _{x \rightarrow \infty} \epsilon(x)=0 .
$$

It follows immediately by Karamata's characterization theorem that $f \in \mathcal{R} \mathcal{V}_{\infty}(\rho)$, iff there exists $x_{0}>0$ such that the function $f$ can be written in the form

$$
f(x)=x^{\rho} \exp \left(\eta(x)+\int_{x_{0}}^{x} \mathrm{~d} t \frac{\epsilon(t)}{t}\right), \quad \forall x \geq x_{0}
$$

in which $\eta$ and $\epsilon$ satisfy (A-4).

Examples of slowly varying functions are $\ln x$ and itterated logarithm $\ln \ln x$; while for $\rho \neq 0, x^{\rho}$ and $x^{\rho} \ln x$ are regularly varying functions of index $\rho$. 


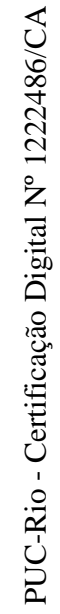




\section{B}

\section{Asymptotic Expansions of Regularized Incomplete Gamma Functions}

The asymptotic expansion of regularized gamma functions defined as

$$
\begin{aligned}
& Q(a, z):=\frac{\Gamma(a, z)}{\Gamma(a)} \\
& P(a, z):=\frac{\gamma(a, z)}{\Gamma(a)}
\end{aligned}
$$

holding uniformly in $\lambda:=z / a>0$, for $a \rightarrow \infty$ and/or $z \rightarrow \infty$, is followed from the representations [103-105]:

$$
\begin{aligned}
& Q(a, z)=\frac{1}{2} \operatorname{erfc}(\eta \sqrt{a / 2})+R_{a}(\eta) \\
& P(a, z)=\frac{1}{2} \operatorname{erfc}(-\eta \sqrt{a / 2})-R_{a}(\eta)
\end{aligned}
$$

with

$$
R_{a}(\eta) \sim \frac{e^{-\frac{1}{2} a \eta^{2}}}{\sqrt{2 \pi a}} \sum_{k=0}^{\infty} c_{k}(\eta) a^{-k}, \quad a \rightarrow \infty,|\arg (a)|<\pi, \quad \text { (uniformly for } \lambda \geq 0 \text { ). }
$$

Here, $\frac{1}{2} \eta^{2}=\lambda-1-\ln \lambda$ with $\operatorname{sgn}(\eta)=\operatorname{sgn}(\lambda-1)$. The coefficients $c_{k}$ in (B-3) are given by

$$
\begin{aligned}
c_{0}(\eta) & =\frac{1}{\mu}-\frac{1}{\eta} \\
\eta c_{k}(\eta) & =\frac{\mathrm{d}}{\mathrm{d} \eta} c_{k-1}(\eta)+(-1)^{k} g_{k} \frac{\eta}{\lambda-1}, \quad k=1,2, \ldots
\end{aligned}
$$

where $g_{k}$ 's are the coefficients that appear in the asymptotic expansion of gamma function

$$
\Gamma(z) \sim \sqrt{2 \pi} e^{-z} z^{z+1 / 2} \sum_{k=0}^{\infty} g_{k} z^{-k}, \quad z \rightarrow \infty .
$$

When $\lambda \neq 1$, the error functions are dominant terms in (B-2) as $a$ tends to infinity. Thus, 
Appendix B. Asymptotic Expansions of Regularized Incomplete Gamma Functions

$$
Q(a, z)=\frac{\Gamma(a, z)}{\Gamma(a)} \sim \frac{1}{2} \operatorname{erfc}(\operatorname{sgn}(\lambda-1) \sqrt{a(\lambda-1-\ln \lambda)}), \quad a \rightarrow \infty .
$$

(Hint: We notice that $\lambda-\ln \lambda-1 \geq 0, \forall \lambda \in \mathbb{R}^{+}$, therefore the argument of the error function in (B-7) is real.)

When $\lambda=1$, we have $\eta=0$ and

$$
Q(a, a)=\frac{\Gamma(a, a)}{\Gamma(a)} \sim \frac{1}{2}+\frac{1}{\sqrt{2 \pi a}} \sum_{k=0}^{\infty} c_{k}(0) a^{-k}, \quad a \rightarrow \infty .
$$




\section{C}

\section{Euler-MacLaurin Summation Formula}

Euler-Maclaurin formula provides a powerful connection between integrals and sums. It can be used to approximate integrals by finite sums, or conversely, to evaluate finite sums and infinite series using integrals. In particular, many asymptotic expansions are derived from this formula. In the most general form, for a $M$ times continuously differentiable function $f$ in the interval $[a, b]\left(f \in C^{M}[a, b]\right)$, this formula can be written as $[106,107]$

$$
\sum_{n=a}^{b} g(n)=\int_{a}^{b} \mathrm{~d} \eta g(\eta)+\frac{1}{2}[g(a)+g(b)]+\sum_{k=1}^{M} \frac{B_{2 k}}{(2 k) !}\left[g^{(2 k-1)}(b)-g^{(2 k-1)}(a)\right]+R_{M} .
$$

The level of approximation in the Euler-Maclaurin formula largely depends on the asymptotic behavior of the remainder term, given by Poisson formula:

$$
R_{M}=\frac{1}{(2 M+1) !} \int_{a}^{b} \mathrm{~d} \eta B_{2 M+1}(\{\eta\}) f^{(2 M+1)}(\eta)
$$

in which $\{\eta\}$ is the fractional part of $\eta$, defined as

$$
\{\eta\}:=\eta-\lfloor\eta\rfloor
$$

and the periodic Bernoulli functions $\widetilde{B}_{n}(\eta) \equiv B_{n}(\{\eta\})$ have the following Fourier series representation, due to Hurwitz:

$$
\widetilde{B}_{n}(\eta)=-\frac{n !}{(2 \pi i)^{n}} \sum_{k=-\infty}^{\infty} \frac{e^{2 \pi i k \eta}}{k^{n}} .
$$

For $a=1, b=N$ and $g(n)=: f(n / N)$ this formula yields:

$$
\begin{aligned}
\frac{1}{N} \sum_{n=1}^{N} f(n / N)=\int_{1 / N}^{1} \mathrm{~d} \xi f(\xi)+ & \frac{1}{2 N}[f(1 / N)+f(1)]+ \\
& \sum_{k=1}^{\infty} \frac{B_{2 k}}{(2 k) ! N^{2 k}}\left[f^{(2 k-1)}(1)-f^{(2 k-1)}(1 / N)\right]
\end{aligned}
$$

which in the limit $N \rightarrow \infty$ reduces to the well-known formula

$$
\lim _{N \rightarrow \infty} \frac{1}{N} \sum_{n=1}^{N} f(n / N)=\int_{0}^{1} \mathrm{~d} \xi f(\xi)
$$


in elementary calculus. ${ }^{1}$ When $N$ is large, but finite, the remaining terms in (C-3) are finite- $N$ corrections to the corresponding summation.

The Mathematica manual [108] reveals that it actually uses Euler-Maclaurin summation formula to do summs like (9-17).

${ }^{1}$ In the limit $N \rightarrow \infty$, the lower limit of the integral in the right hand side of (C-3) can be replaced by zero, provided that the integral exists. 


\section{Bibliography}

[1] WISHART, J.. The generalized product moment distribution in samples from a normal multivariate population, biometrika, a20, 32-52. Anwar H Joarder Department of Mathematical Sciences King Fahd University of Petroleum and Minerals Dhahran, 31261, 1928.

[2] WIGNER, E. P.. On the statistical distribution of the widths and spacings of nuclear resonance levels. In: MATHEMATICAL PROCEEDINGS OF THE CAMBRIDGE PHILOSOPHICAL SOCIETY, volumen 47, p. 790-798. Cambridge University Press, 1951.

[3] DYSON, F. J.. Statistical theory of the energy levels of complex systems. i. Journal of Mathematical Physics, 3(1):140-156, 1962.

[4] DYSON, F. J.. Statistical theory of the energy levels of complex systems. ii. Journal of Mathematical Physics, 3(1):157-165, 1962.

[5] DYSON, F. J.. Statistical theory of the energy levels of complex systems. iii. Journal of Mathematical Physics, 3(1):166-175, 1962.

[6] DYSON, F. J.; MEHTA, M. L.. Statistical theory of the energy levels of complex systems. iv. Journal of Mathematical Physics, 4(5):701-712, 1963.

[7] MEHTA, M. L.; DYSON, F. J.. Statistical theory of the energy levels of complex systems. v. Journal of Mathematical Physics, 4(5):713-719, 1963.

[8] EDELMAN, A.; WANG, Y.. Random matrix theory and its innovative applications. In: ADVANCES IN APPLIED MATHEMATICS, MODELING, AND COMPUTATIONAL SCIENCE, p. 91-116. Springer, 2013.

[9] ATKIN, M. R.; ZOHREN, S.. Violations of bell inequalities from random pure states. Physical Review A, 92(1):012331, 2015.

[10] BEENAKKER, C. W.. Random-matrix theory of quantum transport. Reviews of modern physics, 69(3):731, 1997.

[11] BOHIGAS, O.; GIANNONI, M.-J. ; SCHMIT, C.. Characterization of chaotic quantum spectra and universality of level fluctuation laws. Physical Review Letters, 52(1):1, 1984. 
[12] TULINO, A. M.; VERDÚ, S. ; OTHERS. Random matrix theory and wireless communications. Foundations and Trends $₫$ in Communications and Information Theory, 1(1):1-182, 2004.

[13] EYNARD, B.; KIMURA, T. ; RIBAULT, S.. Random matrices. arXiv preprint arXiv:1510.04430, 2015.

[14] AKEMANN, G.; ATKIN, M. R.. Higher order analogues of tracy-widom distributions via the lax method. Journal of Physics A: Mathematical and Theoretical, 46(1):015202, 2012.

[15] ANDREI, N.. Kolmogorov. foundations of the theory of probability, 1950.

[16] FELLER, W.. An Introduction to Probability Theory and Its Applications. Número v. 1 em An Introduction to Probability Theory and Its Applications. Wiley, 1967.

[17] FELLER, W.. William. an introduction to probability theory and its applications. vol. ii, 1971.

[18] MEESTER, R.. A natural introduction to probability theory. Springer Science \& Business Media, 2008.

[19] ROSS, S. M.. Introduction to probability models. Academic press, 2014.

[20] JACOD, J.; PROTTER, P.. Probability essentials. Springer Science \& Business Media, 2004.

[21] PATRICK, B.. Convergence of probability measures, 1999.

[22] GRIMMETT, G.; WELSH, D.. Probability: an introduction. Oxford University Press, 2014.

[23] GRIMMETT, G.; STIRZAKER, D.. Probability and random processes. Oxford university press, 2001.

[24] PHELPS, R. R.. Convex functions, monotone operators and differentiability. Lecture notes in mathematics, 1364:378, 1989.

[25] ARNOL'D, V. I.. Mathematical methods of classical mechanics, volumen 60. Springer Science \& Business Media, 2013.

[26] FOSS, S.; KORSHUNOV, D.; ZACHARY, S. ; OTHERS. An introduction to heavy-tailed and subexponential distributions, volumen 6. Springer, 2011. 
[27] ASMUSSEN, S.. Applied probability and queues, volumen 51. Springer Science \& Business Media, 2008.

[28] COLES, S.; BAWA, J.; TRENNER, L. ; DORAZIO, P.. An introduction to statistical modeling of extreme values, volumen 208. Springer, 2001.

[29] GUMBEL, E. J.. Statistics of extremes. Courier Corporation, 2012.

[30] DE HAAN, L.; FERREIRA, A.. Extreme value theory: an introduction. Springer Science \& Business Media, 2007.

[31] GELUK, J.; DE HAAN, L.. Stable probability distributions and their domains of attraction: a direct approach. PROBABILITY AND MATHEMATICAL STATISTICS-WROCLAW UNIVERSITY, 20(1):169-188, 2000.

[32] SAlVAdORI, G.; De MiChEle, C.; KOTTEgOdA, N. T. ; ROSSO, R.. Extremes in nature: an approach using copulas, volumen 56 . Springer Science \& Business Media, 2007.

[33] FISHER, R. A.; TIPPETT, L. H. C.. Limiting forms of the frequency distribution of the largest or smallest member of a sample. In: MATHEMATICAL PROCEEDINGS OF THE CAMBRIDGE PHILOSOPHICAL SOCIETY, volumen 24, p. 180-190. Cambridge Univ Press, 1928.

[34] GNEDENKO, B.. Sur la distribution limite du terme maximum d'une serie aleatoire. Annals of mathematics, p. 423-453, 1943.

[35] LeAdBetTER, M. R.; Lindgren, G. ; ROOTZÉN, H.. Extremes and related properties of random sequences and processes. Springer Science \& Business Media, 2012.

[36] KOTZ, S.; NADARAJAH, S.. Extreme value distributions: theory and applications. World Scientific, 2000.

[37] RESNICK, S. I.. Extreme values, regular variation and point processes. Springer, 2013.

[38] EMBRECHTS, P.; KLUPPELBERG, C. ; MIKOSCH, T.. Modelling extremal events. British Actuarial Journal, 5(2):465-465, 1999.

[39] VON MISES, R.. La distribution de la plus grande de $\mathbf{n}$ valeurs, vol. 2 of selected papers of richard von mises. Providence, RI: American Mathematical Society, pp. 271r, 294, 1936.

[40] WEYL, H.. The classical groups: their invariants and representations. Princeton university press, 2016. 
[41] MEHTA, M. L.. Random matrices, volumen 142. Academic press, 2004.

[42] MACDONALD, I. G.. Symmetric functions and Hall polynomials. Oxford university press, 1998.

[43] WIGNER, E. P.. Characteristic vectors of bordered matrices with infinite dimensions i. In: THE COLLECTED WORKS OF EUGENE PAUL WIGNER, p. 524-540. Springer, 1993.

[44] WIGNER, E. P.. Characteristic vectors of bordered matrices with infinite dimensions ii. In: THE COLLECTED WORKS OF EUGENE PAUL WIGNER, p. 541-545. Springer, 1993.

[45] WIGNER, E. P.. Statistical properties of real symmetric matrices with many dimensions. Princeton University, 1959.

[46] WIGNER, E. P.. On the distribution of the roots of certain symmetric matrices. Annals of Mathematics, p. 325-327, 1958.

[47] BESSIS, D.; ITZYKSON, C. ; ZUBER, J.-B.. Quantum field theory techniques in graphical enumeration. Advances in Applied Mathematics, 1(2):109-157, 1980.

[48] MOROZOV, A.. Matrix models as integrable systems. In: PARTICLES AND FIELDS, p. 127-210. Springer, 1999.

[49] LANDO, S. K.; ZVONKIN, A. K.. Graphs on surfaces and their applications, volumen 2. Springer, 2004.

[50] PASQUALE, A.. Weyl's integration formula on $\mathbf{u}(\mathbf{n})$. In: SUMMER SCHOOL, 2000.

[51] ZYCZKOWSKI, K.; OTHERS. Hilbert-schmidt volume of the set of mixed quantum states. Journal of Physics A: Mathematical and General, 36(39):10115, 2003.

[52] ANDERSON, G. W.; GUIONNET, A. ; ZEITOUNI, O.. An introduction to random matrices, volumen 118. Cambridge university press, 2010.

[53] MARINO, M.. Les houches lectures on matrix models and topological strings. arXiv preprint hep-th/0410165, 2004.

[54] ESTRADA, R.; KANWAL, R. P.. Singular integral equations. Springer Science \& Business Media, 2012. 
[55] MUSKHELISHVILI, N.. Singular integral equations: boundary problems of function theory and their application to mathematical physics.

[56] ABLOWITZ, M. J.; FOKAS, A. S.. Complex variables: introduction and applications. Cambridge University Press, 2003.

[57] ARFKEN, G. B.; WEBER, H. J. ; HARRIS, F. E.. Mathematical methods for physicists: a comprehensive guide. Academic press, 2011.

[58] BRÉZIN, E.; ITZYKSON, C.; PARISI, G. ; ZUBER, J.-B.. Planar diagrams. Communications in Mathematical Physics, 59(1):35-51, 1978.

[59] DEIFT, P.. Orthogonal polynomials and random matrices: a RiemannHilbert approach, volumen 3. American Mathematical Soc., 2000.

[60] NADAL, C.; MAJUMDAR, S. N.. A simple derivation of the tracywidom distribution of the maximal eigenvalue of a gaussian unitary random matrix. Journal of Statistical Mechanics: Theory and Experiment, 2011(04):P04001, 2011.

[61] SZEG, G.. Orthogonal polynomials, volumen 23. American Mathematical Soc., 1939.

[62] FYODOROV, Y. V.; SOMMERS, H.. Random matrices close to hermitian or unitary: overview of methods and results. Journal of Physics $A$ : Mathematical and General, 36(12):3303, 2003.

[63] HAAKE, F.; IZRAILEV, F.; LEHMANN, N.; SAHER, D. ; SOMMERS, H.-J.. Statistics of complex levels of random matrices for decaying systems. Zeitschrift für Physik B Condensed Matter, 88(3):359-370, 1992.

[64] GARCIA-GARCIA, A.; NISHIGAKI, S. ; VERBAARSCHOT, J.. Critical statistics for non-hermitian matrices. Physical Review E, 66(1):016132, 2002.

[65] GINIBRE, J.. Statistical ensembles of complex, quaternion, and real matrices. Journal of Mathematical Physics, 6(3):440-449, 1965.

[66] STEPHANOV, M. A.. Random matrix model of qcd at finite density and the nature of the quenched limit. Physical Review Letters, 76(24):4472, 1996.

[67] GROBE, R.; HAAKE, F. ; SOMMERS, H.-J.. Quantum distinction of regular and chaotic dissipative motion. Physical review letters, 61(17):1899, 1988. 
[68] DI FRANCESCO, P.; GAUDIN, M.; ITZYKSON, C. ; LESAGE, F.. Laughlin's wave functions, coulomb gases and expansions of the discriminant. International Journal of Modern Physics A, 9(24):4257-4351, 1994.

[69] BURDA, Z.; JANIK, R. ; WACLAW, B.. Spectrum of the product of independent random gaussian matrices. Physical Review E, 81(4):041132, 2010.

[70] BURDA, Z.; JANIK, R. ; NOWAK, M.. Multiplication law and s transform for non-hermitian random matrices. Physical Review E, 84(6):061125, 2011.

[71] SOMMERS, H.; CRISANTI, A.; SOMPOLINSKY, H. ; STEIN, Y.. Spectrum of large random asymmetric matrices. Physical review letters, 60(19):1895, 1988.

[72] GIRKO, V. L.. Spectral theory of random matrices. Russian Mathematical Surveys, 40(1):77-120, 1985

[73] FYODOROV, Y. V.; SOMMERS, H.-J.. Statistics of resonance poles, phase shifts and time delays in quantum chaotic scattering: Random matrix approach for systems with broken time-reversal invariance. Journal of Mathematical Physics, 38(4):1918-1981, 1997.

[74] JANIK, R. A.; NOWAK, M. A.; PAPP, G. ; ZAHED, I.. Non-hermitian random matrix models. Nuclear Physics B, 501(3):603-642, 1997.

[75] ZOHREN, S.. A causal perspective on random geometry. arXiv preprint arXiv:0905.0213, 2009.

[76] AMBJØRN, J.; DURHUUS, B.. Quantum geometry: a statistical field theory approach. Cambridge University Press, 1997.

[77] BURDA, Z.; GÖRLICH, A.; JAROSZ, A. ; JURKIEWICZ, J.. Signal and noise in correlation matrix. Physica A: Statistical Mechanics and its Applications, 343:295-310, 2004.

[78] T HOOFT, G.. A planar diagram theory for strong interactions. The Large N Expansion in Quantum Field Theory and Statistical Physics. Edited by BREZIN E ET AL. Published by World Scientific Publishing Co. Pte. Ltd., 1993. ISBN\# 9789814365802, pp. 80-92, p. 80-92, 1993.

[79] CHAU, L.-L.; YU, Y.. Unitary polynomials in normal matrix models and wave functions for the fractional quantum hall effects. Physics Letters A, 167(5-6):452-458, 1992. 
[80] MACKLIN, P. A.. Normal matrices for physicists. American Journal of Physics, 52(6):513-515, 1984.

[81] SAFF, E. B.; TOTIK, V.. Logarithmic potentials with external fields, volumen 316. Springer Science \& Business Media, 2013.

[82] ZABRODIN, A.. New applications of non-hermitian random matrices. In: INTERNATIONAL CONFERENCE ON THEORETICAL PHYSICS, p. 851861. Springer, 2003.

[83] CUNDEN, F. D.; MALTSEV, A. ; MEZZADRI, F.. Fluctuations in the two-dimensional one-component plasma and associated fourth-order phase transition. Physical Review E, 91(6):060105, 2015.

[84] ELBAU, P.. Random normal matrices and polynomial curves. arXiv preprint arXiv:0707.0425, 2007.

[85] TRACY, C. A.; WIDOM, H.. Level-spacing distributions and the airy kernel. Communications in Mathematical Physics, 159(1):151-174, 1994.

[86] TRACY, C.; WIDOM, H.. On orthogonal and symplectic matrix ensembles, 1996. Commun. Math. Phys, 177:727.

[87] TRACY, C. A.; WIDOM, H.. Distribution functions for largest eigenvalues and their applications. arXiv preprint math-ph/0210034, 2002.

[88] BOROT, G.; EYNARD, B.; MAJUMDAR, S. N. ; NADAL, C.. Large deviations of the maximal eigenvalue of random matrices. Journal of Statistical Mechanics: Theory and Experiment, 2011(11):P11024, 2011.

[89] BOROT, G.; NADAL, C.. Right tail asymptotic expansion of tracywidom beta laws. Random Matrices: Theory and Applications, 1(03):1250006, 2012.

[90] ATKIN, M. R.; ZOHREN, S.. Instantons and extreme value statistics of random matrices. arXiv preprint arXiv:1307.3118, 2013.

[91] MAJUMDAR, S. N.; SCHEHR, G.. Top eigenvalue of a random matrix: large deviations and third order phase transition. Journal of Statistical Mechanics: Theory and Experiment, 2014(1):P01012, 2014.

[92] FORRESTER, P. J.. The spectrum edge of random matrix ensembles. Nuclear Physics B, 402(3):709-728, 1993.

[93] DOUGLAS, M. R.; SHENKER, S. H.. Strings in less than one dimension. Nuclear Physics B, 335(3):635-654, 1990. 
[94] BREZIN, E.; KAZAKOV, V.. Exactly solvable field theories of closed strings. Physics Letters B, 236(2):144-150, 1990.

[95] GROSS, D. J.; MIGDAL, A. A.. Nonperturbative two-dimensional quantum gravity. Physical Review Letters, 64(2):127, 1990.

[96] DI FRANCESCO, P.; GINSPARG, P. ; ZINN-JUSTIN, J.. 2d gravity and random matrices. Physics Reports, 254(1-2):1-133, 1995.

[97] DAS, A.. Integrable models, volumen 30. World scientific, 1989.

[98] RIDER, B.. Order statistics and ginibre's ensembles. Journal of statistical physics, 114(3):1139-1148, 2004

[99] CHAFAÏ, D.; PÉCHÉ, S.. A note on the second order universality at the edge of coulomb gases on the plane. Journal of Statistical Physics, 156(2):368-383, 2014.

[100] KARAMATA, J.. Sur un mode de croissance reguliere des fonctions, mathematica4 (1930), 38-53. Karamata384Mathematica1930.

[101] BINGHAM, N. H.; GOLDIE, C. M. ; TEUGELS, J. L.. Regular variation, volumen 27. Cambridge university press, 1989.

[102] SENETA, E.. Regularly varying functions. 1977.

[103] TEMME, N.. Uniform asymptotic expansions of the incomplete gamma functions and the incomplete beta function. Mathematics of Computation, 29(132):1109-1114, 1975.

[104] TEMME, N. M.. Special functions: An introduction to the classical functions of mathematical physics. John Wiley \& Sons, 2011.

[105] GAUTSCHI, W.. The incomplete gamma functions since tricomi. In: IN TRICOMI'S IDEAS AND CONTEMPORARY APPLIED MATHEMATICS, ATTI DEI CONVEGNI LINCEI, N. 147, ACCADEMIA NAZIONALE DEI LINCEI. Citeseer, 1998.

[106] ABRAMOWITZ, M.; STEGUN, I. A.. Handbook of mathematical functions: with formulas, graphs, and mathematical tables, volumen 55. Courier Corporation, 1964.

[107] GAUTSCHI, W.. Numerical analysis. Springer Science \& Business Media, 2011.

[108] WOLFRAM, S.. The Mathematica. Cambridge university press Cambridge, 1999. 